\title{
Article \\ From 5G to 6G Technology: Meets Energy, Internet-of-Things and Machine Learning: A Survey
}

\author{
Mohammed Najah Mahdi ${ }^{1, *(\mathbb{D}}$, Abdul Rahim Ahmad ${ }^{2}$, Qais Saif Qassim ${ }^{3} \mathbb{D}_{\text {, Hayder Natiq }}{ }^{4}$ (D), \\ Mohammed Ahmed Subhi ${ }^{5}$ and Moamin Mahmoud ${ }^{1}$ (D) \\ 1 Institute of Informatics and Computing in Energy, Universiti Tenaga Nasional, Kajang 43000, Malaysia; \\ moamin@uniten.edu.my \\ 2 College of Computing and Informatics (CCI), Universiti Tenaga Nasional, Kajang 43000, Malaysia; \\ Abdrahim@uniten.edu.my \\ 3 College of Technology, University of Technology and Applied Sciences, Ibri 511, Oman; \\ qais.aljanabi@ibrict.edu.om \\ 4 Department of Computer Technology, Information Technology Collage, Imam Ja'afar Al-Sadiq University, \\ Baghdad 10064, Iraq; hayder.natiq@sadiq.edu.iq \\ 5 Department of Computer Engineering Techniques, Al Hikma University College, Baghdad 10064, Iraq; \\ mohammed.subhi@hiuc.edu.iq \\ * Correspondence: Najah.Mahdi@uniten.edu.my
}

Citation: Mahdi, M.N.; Ahmad, A.R.; Qassim, Q.S.; Natiq, H.; Subhi, M.A.;

Mahmoud, M. From 5G to 6G

Technology: Meets Energy, Internet-of-Things and Machine Learning: A Survey. Appl. Sci. 2021

11, 8117. https://doi.org/

10.3390/app11178117

Academic Editor: Florina Ludosan

Received: 15 July 2021

Accepted: 17 August 2021

Published: 31 August 2021

Publisher's Note: MDPI stays neutral with regard to jurisdictional claims in published maps and institutional affiliations.

Copyright: (c) 2021 by the authors. Licensee MDPI, Basel, Switzerland. This article is an open access article distributed under the terms and conditions of the Creative Commons Attribution (CC BY) license (https:// creativecommons.org/licenses/by/ $4.0 /)$
Abstract: Due to the rapid development of the fifth-generation (5G) applications, and increased demand for even faster communication networks, we expected to witness the birth of a new 6G technology within the next ten years. Many references suggested that the $6 \mathrm{G}$ wireless network standard may arrive around 2030. Therefore, this paper presents a critical analysis of $5 \mathrm{G}$ wireless networks', significant technological limitations and reviews the anticipated challenges of the $6 \mathrm{G}$ communication networks. In this work, we have considered the applications of three of the highly demanding domains, namely: energy, Internet-of-Things (IoT) and machine learning. To this end, we present our vision on how the $6 \mathrm{G}$ communication networks should look like to support the applications of these domains. This work presents a thorough review of 370 papers on the application of energy, IoT and machine learning in 5G and 6G from three major libraries: Web of Science, ACM Digital Library, and IEEE Explore. The main contribution of this work is to provide a more comprehensive perspective, challenges, requirements, and context for potential work in the $6 \mathrm{G}$ communication standard.

Keywords: 5G; 6G; energy; deep learning; machine learning; Internet-of-Things; IoT; security

\section{Introduction}

Many researchers have anticipated that the year 2030 will bring tremendous changes in technology and business landscapes [1]. The world is moving towards a data-driven, highly digitalised and intelligent environment. The new revolution will introduce new technological challenges and requirements. In order to cope with these technical requirements, the existing communication networks should be improved and enhanced. The sixth-generation (6G) mobile communication network is expected to play a vital role in supporting the required connection speed, reliability, coverage and infrastructure in the future. Based on existing reports, the sixth generation will furnish a full dimensional wireless range and support all industrial functions, including sensing, communication, computation, caching, control, location, radar, navigation, and imaging full-vertical applications. Recent academic articles have been conceptualizing the $6 \mathrm{G}$ to be a self-contained ecosystem with intelligence and decision making skills comparable to humans. It is expected that mobile communication will progress from being human-centric to being both human/machinecentric. Moreover, it will brace various communication methods to interact with intelligent end-points; also supporting various biometric-based applications including fingerprints, 
voice recognition, eye-tracking, and brainwaves. The $6 \mathrm{G}$ mobile communication networks are expected to offer up to 100 per cent gain in energy efficiency over their predecessors, the 5G networks, and a highly complex structure due to vast interconnections. Statistical studies show that there will be an increase of mobile data traffic at a rate of 55 per cent annually [2]. By the year 2030, that generated traffic will cross five zettabytes $\left(10^{21}\right)$ every month. With this massive volume of data generated, there should be a mechanism to handle and manage the network at various levels. To this end, the application of Artificial Intelligence (AI) will be heavily dependent on the next-generation wireless network. It is interesting to consider how the Internet-of-Things (IoT) enabled devices will communicate [3]. IoT is a crucial technology for attaining the "social information infrastructure" [4], where it can be used to visualise and simplify complicated and mutually related societal problems in the real world. In this study, we present a critical analysis of 5G wireless networks' significant technological limitations with respect to the expected advancement in three major domains: energy, Internet-of-Things, and machine learning. Moreover, the study also presents a review of the anticipated challenges of the $6 \mathrm{G}$ communication networks. The overall contribution is presenting a visionary framework on how the 6G should look like to support the applications of these domains.

Contribution: This article aims to draw a complete picture of "how the $5 \mathrm{G}$ to $6 \mathrm{G}$ Technology: meets Energy, Internet-of-Things and Machine Learning?".

We cover different dimensions and aspects of $5 \mathrm{G}$ and $6 \mathrm{G}$ focusing on the projected 5G and 6G system architecture, potential technologies, an overview of an existing technique, analysis of ML usage in incorporating energy and IoT in 5G and 6G, applications, and use cases. The taxonomy of the paper is shown in Figure 1, which gives a pictorial view of all sections and subsections presented in this paper. The contributions of this article are summarized as follows.

- We discuss in detail the projected 6G system architecture. Also, the existing research literature reveals that providing full-dimensional advantages of the 6G technology; will 5G application types will be reconsidered by modifying the traditional URLLC, eMBB, and mMTC and providing new services.

- We discuss the primary aims, vision, and trends for 6G network dimensions that include our vision of $6 \mathrm{G}$ and IoT and machine learning applications for autonomous networks and revolutionary energy efficiency, which are discussed in detail throughout this paper.

- We highlight all the essential network elements of 6G system architecture and discuss defined issues that current generations of mobile networks are facing; the mobile industry should transition away from traditional strategies and toward some new ones, such as operation in shared spectrum bands, inter-operator spectrum sharing, indoor small cell networks, a large number of local network operators, and on-demand network slice leasing.

- To meet the requirements of the stage where the research results on ML are to be addressed, as well as the methodology used by $5 \mathrm{G}$ and $6 \mathrm{G}$ for the Internet of Things and energy transmissions. We overview six major categories, including network resource management, security, augmented reality, network scaling, resource allocation, and the smart grid.

- Towards the end, we discuss multiple challenges and research directions applications, issues, research questions, motivation, recommendation criteria, and open challenges of ML usage in incorporating energy and IoT in 5G and 6G, 


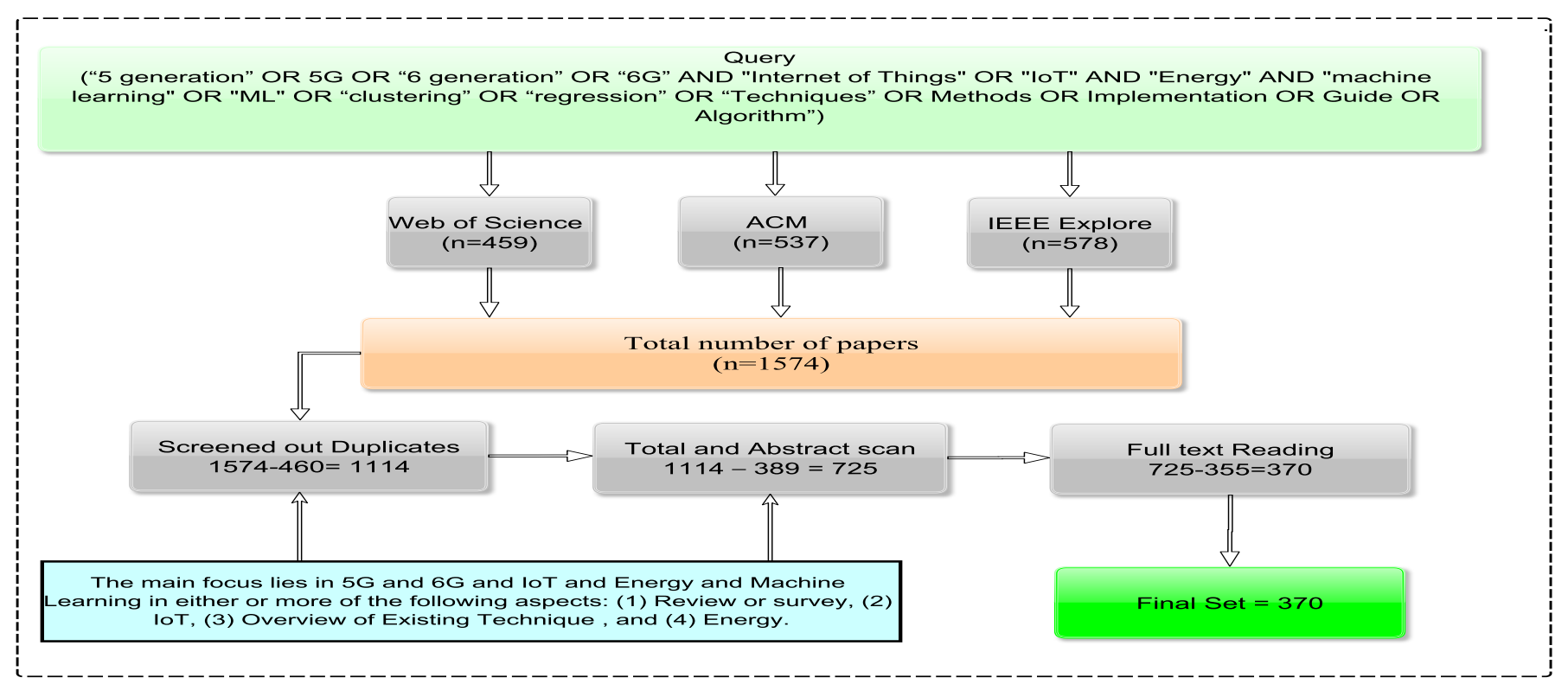

Figure 1. Research Methodology Guideline.

The remaining paper is outlined as follows. Section 2 defines the approach, including the source of material, requirements for eligibility of research, the systematic literature review (SLR) and the effects of search results of publications. The queries of any object from three website papers separated into 4 classes, the literary taxonomy on $5 \mathrm{G}$ and $6 \mathrm{G}$ Technology. Section 3 We discuss in detail about the projected 6G system architecture. We highlight all the essential network elements of $6 \mathrm{G}$ system architecture, application of AI and ML, existing technique, analysis of ML usage in incorporating energy and IoT in 5G and $6 \mathrm{G}$, etc. Section 4 described and summarised in survey and review papers based on the latest state of the art in 5G,6G, and research documentation. Section 5 investigated in this section, the requirements of the stage where the results of the research on ML are to be addressed, as well as the methodology used by 5G and 6G for the Internet of Things and energy transmissions. Section $6 \mathrm{We}^{\prime} \mathrm{ve}$ done an explanatory evaluation of the use of the internet of things as the foundation for a transition strategy toward the development of $5 \mathrm{G}$ and $6 \mathrm{G}$ communications networks and technologies. Section 7 to handle various difficulties in wireless communications in the direction of merging energy and IoT in 5G, 6G, and beyond networks. Section 8 addressed inspiration, difficulties, and recommendations in that research area and a modern approach to the $5 \mathrm{G}$ and $6 \mathrm{G}$ networks that make use of IoT, energy and machine learning technologies. Finally, Section 9 presents the Conclusion. The reminder of this paper is organized as illustrated in Figure 2. 


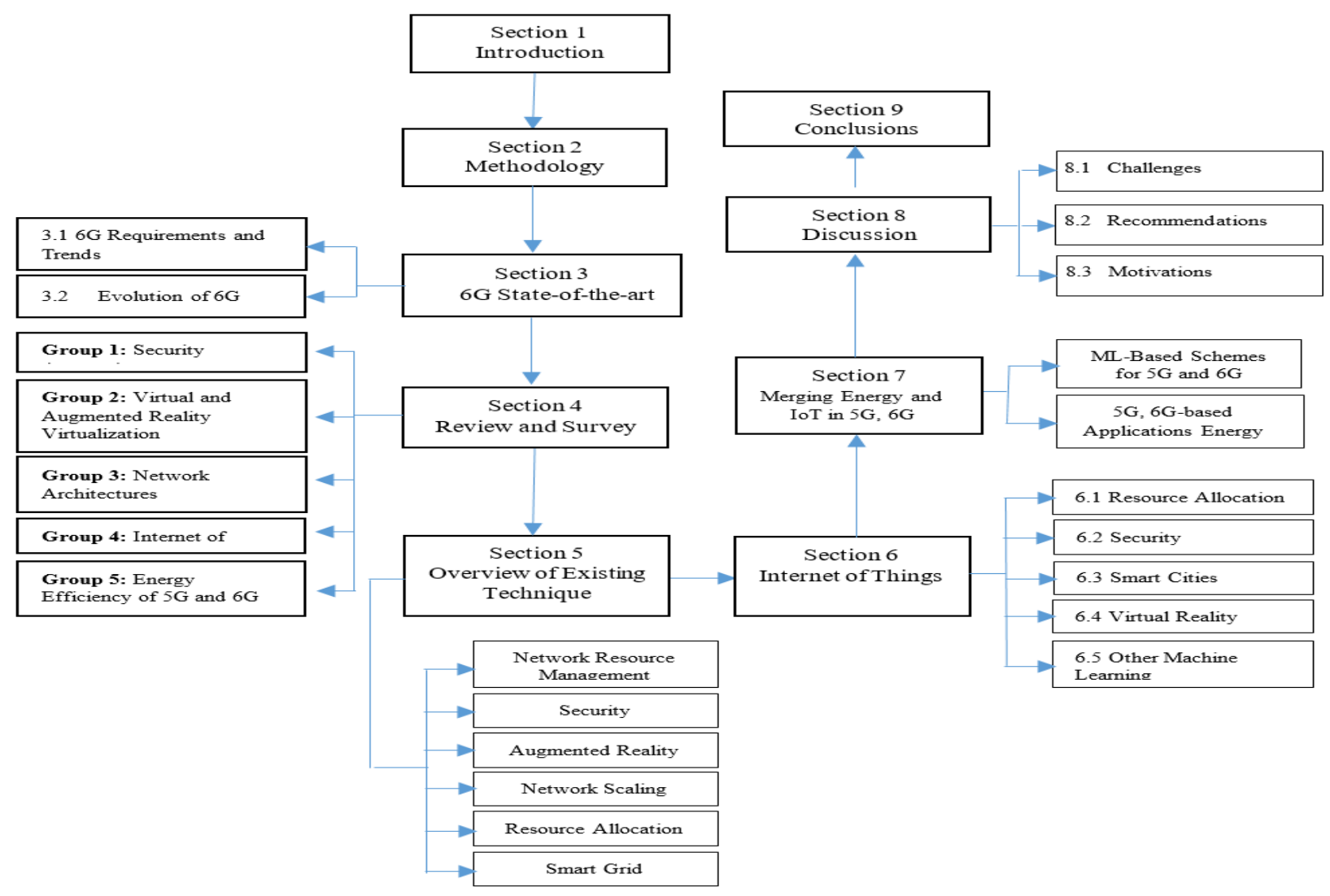

Figure 2. Diagramatic view of the organization of this survey paper.

\section{Methodology}

We found articles about 5G and 6G by using three keywords: "machine learning", "internet of things", and "energy". The searches were conducted using three digital libraries: (1) Web of Science (WOS), which provides multidisciplinary research articles in the fields of science, arts, and technology; (2) IEEE Explore, which provides articles specialised in the field of electrical and electronics engineering; and (3) ACM digital library, which has a comprehensive database of scientific articles related to computing and technology.

The significant articles and literature referred to by the search outputs were selected and categorised based on two criteria: (1) use three iterations in the filtering process, which removes the redundant and duplicated articles, excluding irrelevant articles using the title; and (2) use three iterations in the categorization process, which categorises the significant articles and literature referred by the search outputs. Perform the initial screening, and the selected papers are then subjected to a second selected using the $5 \mathrm{G}$ and $6 \mathrm{G}$ after carefully reviewing the results of the reduced search.

The three databases mentioned were searched extensively in May 2021, with many different keywords (or phrases) being used, including " 5 generations" or " $5 \mathrm{G}$ " or " 6 generations" or "6G" and "machine learning" or ML or "artificial intelligence" or " $\mathrm{AI}$ " or "classification" or "clustering" or "regression", and "IoT" or "Internet of Things" and "Energy" or "techniques" or "methods" or "implementation" or "guide".

Figure 2 depicts an example of a query text that might be utilised. Using the advanced features of search engines, we have removed search results that are correspondences, letters, book chapters, and other types of documents. The exclusions are designed to ensure that only the most recent scientific publications are obtained, as well as just those of significant value that improve the $5 \mathrm{G}$ and $6 \mathrm{G}$ capabilities. The emphasis is on including any articles and scientific submissions that meet all of the requirements for inclusion in this work. Following that, they are separated into two categories, namely, general and coarse-grained classifications. Following the study's findings, the latter is explored in four succeeding 
sections derived from the results, in which the Google scholar search engine was applied to determine the path of the study.

After the queries were run, 1574 papers were found, with 459 coming from WOS, 578 from IEEE, and 537 from ACM digital libraries. Between 2014 and 2021, all of the articles on this list were published. These articles were then sorted into three groups: (1) 460 redundant articles, (2) 744 irrelevant articles based on titles and abstracts, and 370 articles that met the requirements for 5G, 6G, machine learning, internet of things, and energy.

As previously stated, an article is removed from consideration if it fails to meet the following selection criteria: (1) The paper was not written in an English-speaking environment. (2) The article's emphasis was on techniques and/or procedures. (3) The article's research focus is solely on 5G and 6G, with no mention of the internet of things, energy, or machine learning.

Furthermore, if $5 \mathrm{G}$ and $6 \mathrm{G}$ were not added in the second iteration, the articles would still be removed. (1) There are no features of machine learning, IoT, or energy considered in the paper's contribution. (2) The paper's discussion is limited to $5 \mathrm{G}$ and $6 \mathrm{G}$, with no other topics addressed. Articles are subjected to rigorous machine learning, internet of things, and energy analysis in this study, with the remaining articles being sorted into categories that focus on how to improve $5 \mathrm{G}$ and $6 \mathrm{G}$.

Results and Statistical Information of Articles

The review's findings are addressed in the form of responses to the research questions. The taxonomy is shown in Figure 3. There are four basic categories in which the recordings might be classified. (1) Review and Survey (2) Internet of Things (3) Overview of Existing Technique and (4) Energy. The first set of research and survey materials describes the IoT and energy or machine learning methodologies and strategies used in 5G and 6G to achieve their goals and solve challenges. The second section looks at the impacts, triggers, countermeasures, and conditions, as well as technology for better efficacy control. The effects of a methodology used to classify various variables, which can alter different parts of the method or the product as it is generated, are presented in the third category. The fourth category includes a mission's structures, tactics, and low energy usage and operational efficiency.

Figure 3 shows the statistics for the various categories listed above for articles about 5G and 6G. The 370 articles from the three databases are split into four categories in the figure: reviews and surveys (74) [5-78] , Internet of Things (95) [79-139], Overview of Existing Technique (61) [140-234], and publications about Energy studies (140) [235-374]. Figure 3 shows the quick number of publications in 5G and 6G based on the fields and regions where research and studies are conducted. The findings were divided into 74 of the 370 papers, 95 of which are relevant publications on $5 \mathrm{G}$ and $6 \mathrm{G}$ case analysis $5 \mathrm{G}$ and 6G and internet of things strategies, and 61 of the 370 articles, which addressed with the technique used by $5 \mathrm{G}$ for IoT and energy. The last categories of scientific contributions and outcomes focused on combining energy and IoT in 5G, 6G, and 140 of 380 papers. Even the mathematical study of several groups is depicted in the illustration.

Figure 4, on the other hand, presents classified scientific publications from 2014 to 2021 and contains papers based on the year of publication. Only two papers were authored in 2014, yet 170 were published between 2015 and 2018. In comparison, 109, 54, and 35 papers have been written for 2019, 2020, and 2021, respectively. Primary sources of analysis were based on $5 \mathrm{G}$ and $6 \mathrm{G}$ research, and general recommendations were evaluated. 


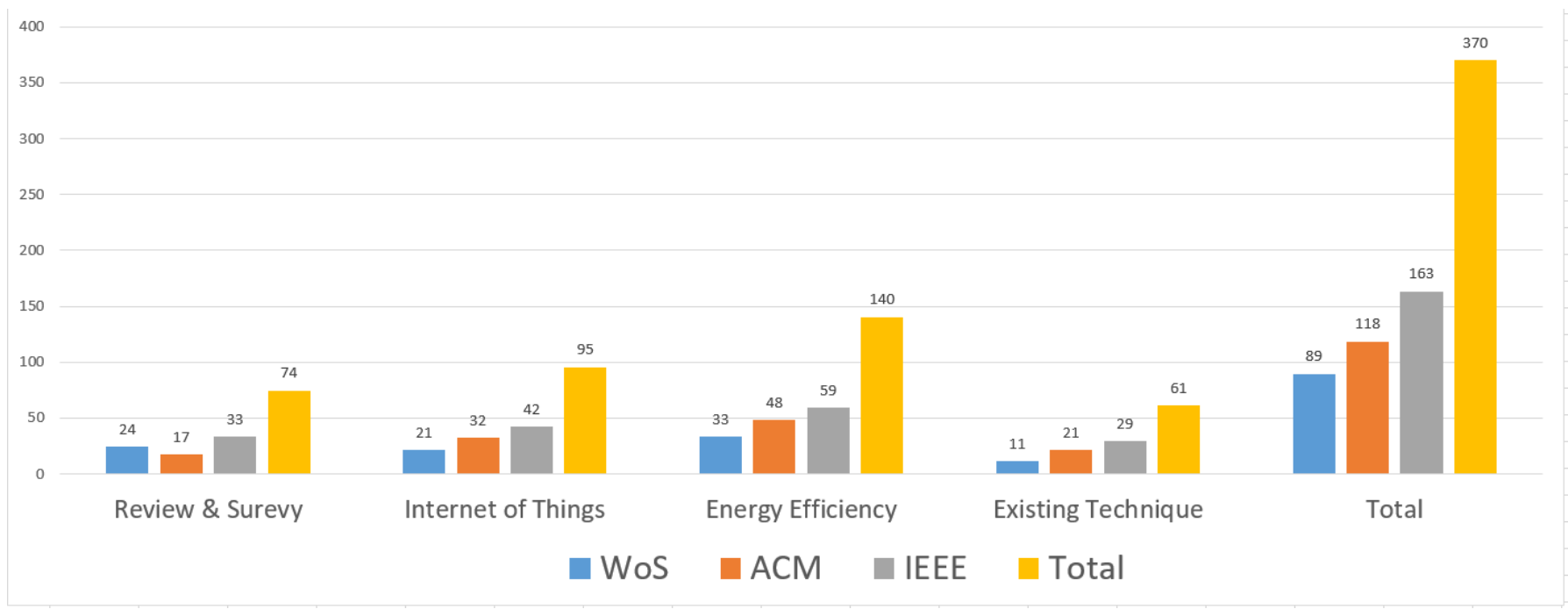

Figure 3. Articles categorized based on their contribution.

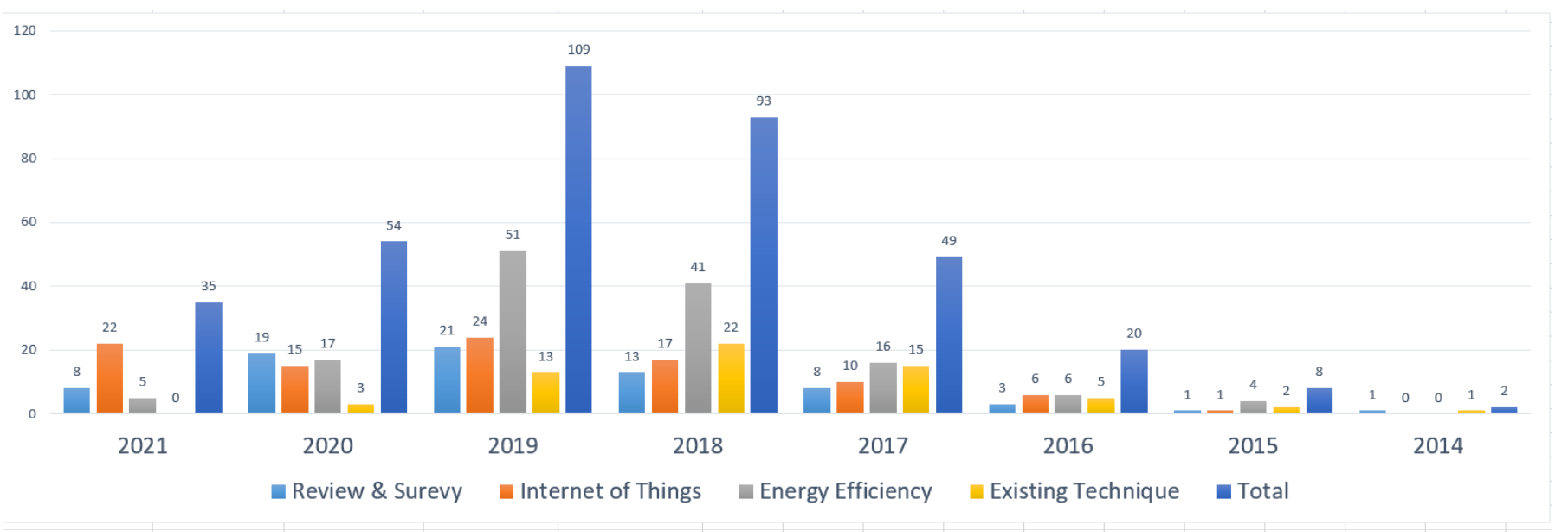

Figure 4. Published articles in between 2014 and 2021.

\section{6G State-of-the-Art}

For this vision to become a reality, $6 \mathrm{G}$ will be the critical enabler; it will connect everything, provide full-dimensional wireless coverage, and integrate all functions, including sensing and communication, as well as computation, caching, and control [375]. It will also enable full-vertical applications by integrating all functions, including as sensing, communication, computation, caching, and control, as well as location, radar, navigation, and imaging, into a single platform. It is anticipated that $6 \mathrm{G}$ would be an autonomous ecosystem with intelligence and consciousness on par with humans. In the coming years, 6G networks will be a significant enabler for the intelligent information society, as they are expected to provide performance superior to $5 \mathrm{G}$ networks while also catering to the needs of developing services and applications. Our vision of $6 \mathrm{G}$, as well as IoT and machine learning applications for autonomous networks and revolutionary energy efficiency, are discussed in detail throughout this paper.

Despite the fact that 5G supports URLLC, Zong et al. [376] argue that it has some limitations, such as the weakness of short packet, sensing-based URLLC. This may limit the dependability of low-latency services with high data rates, which are required for AR, MR, and VR. Furthermore, smart devices are expected to exponentially increase data traffic and necessitate high-speed data transfer, both of which are unaccounted for in 5G 
standards [377]. Similarly, 5G does not support advanced IoT technologies that necessitate the convergence of communication, detection, control, and computing functions. As a result, the need for $6 \mathrm{G}$ emerges to support these IoT technologies. In comparison to 5G, $6 \mathrm{G}$ will be more reliable, have lower latency, and will be fully integrated with $\mathrm{ML}$, $\mathrm{XR}$, IoT, and blockchain technologies [378]. In addition, 6G wireless networks will: (1) With super-high throughput demands, support SHD and EHD videos. (2) Provide extremely low-latency communications for the industrial Internet (about $10 \mathrm{~s}$ ). (3) Support the Internet of Nano-Things and the Internet of Bodies by using smart wearable devices and intraday communications enabled by implantable nanodevices and nanosensors that consume very little power. (4) Support underwater and space communications to considerably expand human activity's bounds, such as deep-sea touring and space exploration. (5) In new scenarios, such as HSR, provide consistent service experiences. (6) Boost 5G vertical applications like Massive IoT and completely autonomous vehicles.

\subsection{G Requirements and Trends}

As a result of the clearly defined issues that current generations of mobile networks are facing, the mobile industry should transition away from traditional strategies and toward some new ones, such as operation in shared spectrum bands, inter-operator spectrum sharing, indoor small cell networks, a large number of local network operators, and ondemand network slice leasing. Several of the most essential criteria and trends for the future generation of mobile networks will be discussed in greater detail in the sections to follow.

\subsubsection{Self-X Network}

The future network must be more adaptive and robust, and it must be capable of managing itself far beyond the capabilities of people. Machine learning approaches that are both intelligent and adaptive are employed to enable 6G networks to be self-sufficient while also capturing insights and comprehension about their surrounding environment. Without the need for human interaction, the future network will be able to perform functions such as learning from its own mistakes, reconfiguring itself to improve its performance, self-healing, organising itself into groups, and protecting itself.

\subsubsection{Superior Energy Efficiency}

6G devices consume much more energy than prior generations of devices due to their capacity to operate in higher frequency bands than earlier generations. Because of this, energy consumption and efficiency are major issues that must be addressed immediately. Nevertheless, when new techniques are developed, we will witness a rise in energy efficiency as well as the possibility of battery-free internet of things devices, such as energy harvesting in building automation and smart homes.

Interconnectivity in three dimensions: Future networks will extend beyond the confines of two dimensions to include oceans, the atmosphere, and space, allowing for the integration of terrestrial and aerial devices. Various applications, including underwater acoustic ad hoc and sensor networks, weather forecasting, and climate monitoring, will be able to take advantage of such networks.

\subsubsection{Satellite Integration}

To achieve worldwide coverage, future $6 \mathrm{G}$ communications will rely on satellite technologies to be implemented. In the future, $6 \mathrm{G}$ will link telecommunications, earth imaging, and navigation satellites to give cellular users with location services, broadcast and Internet connectivity, and forecasting information. One example is the provision of high-speed Internet access aboard fast trains and aeroplanes. 


\subsection{4. e-Health}

The Internet of Things will usher in a new era of healthcare applications by offering real-time haptic input, continuous connection availability, ultra-low-latency data transfer, extremely high reliability, and support for mobility.

\subsubsection{Smart Cityh}

ITS, IMD, and SRS are examples of smart urban applications that require pervasive sensing as well as intelligent decision-makers and actuators. Smart cities include smart transportation, smart grid, urban infrastructure, resident living environment, transportation management, medical treatment, shopping, and security assurance, among other things.

\subsubsection{Smart Home}

In the future, intelligent houses will be able to provide consumers with comprehensive services such as energy management, patient support, real-time product labelling, and subscription management, among other features.

\subsection{Evolution of $6 G$}

In order to make use of the new technological advantages of 6G technology, 5G application types will be reconsidered by modifying the traditional URLLC, eMBB, and mMTC and providing new services, which are explained below.

\subsubsection{Mobile Broadband Reliable Low-Latency}

The distinction between eMBB and URLLC will become unsustainable for some applications, such as augmented reality, virtual reality, and wireless brain computing interfaces, because these applications require not just excellent reliability but also data throughput on the order of eMBB. As a result, a new service class called MBRLLC was created to enable 6G technology to achieve any desired performance with great dependability and minimal latency.

\subsubsection{MURLLC}

6G technology must scale the typical URLLC feature of 5G into a new service called mURLLC, which offers a high-reliability scalability-latency trade-off in comparison to average-based network designs.

\subsubsection{HCSs}

A new level of physical experience may be delivered with the use of $6 \mathrm{G}$ technology, which enables the provision of HCS services that can be tightly tied with human users. As an excellent example of HCS, wireless brain computing interfaces are particularly well-suited since network performance is determined by the physiology and behaviour of human users.

The major needs for 6G systems are high efficiency, smooth integration, innovative technologies, accurate indoor location, high density, advanced connectivity, and healthy communication. 6G systems must also meet the following characteristics. These more advanced requirements necessitate the provision of technical help that is more innovative in nature. Following is a breakdown of the existing techniques in this field into four (4) groups, as seen in the table below.

(1) Offloading energy-conscious tasks the majority of IoT devices are battery-powered and have limited computing and communication capabilities. As a result, these gadgets can only perform a limited number of functions at a slow rate. On the other side, applications are becoming more computationally intensive, necessitating reduced latencies and realtime answers. These apps might quickly consume the devices' power resources, making them unavailable for usage in the network. Extending the lifetime of IoT devices by conserving/boosting device energy to make full use of their finite energy supplies is still a major challenge [379]. 
(2) Massive IoT: this term refers to the connectivity of a large number of devices, sensors, and equipment on a big scale [380,381]. The Internet of Things is classified according to the number of connected devices and the volume of traffic generated by such devices. The number of devices linked to the Internet has expanded dramatically during the last decade. This number will more than double in the next several years, necessitating the establishment of a robust network to enable their connectivity [382]. The network density in huge IoT is extraordinarily high, reaching roughly 1 million devices per square kilometre [383]. This will result in a significant amount of data being distributed between devices, a large number of tasks being performed, and massive volumes of data being saved and analyzed [384]. As a result, 6G and fog computing will be critical facilitators of huge IoT applications.

(3) High Efficiency: refers to a broad issue that incorporates high data transmission rates, high capacity, large-scale data processing capability, exact research and conclusions, and more.

Dynamic Spectrum Allocation: High flexibility is required for 6G networks to achieve significant efficiency. When it comes to dynamic spectrum allocation, the flexible use of perceptual context information, the rapid allocation of spectrum resources in a matter of seconds, and the intensive use of valuable spectrum resources as a final goal are all possible requirements.

Optimisation of the supply of any service to a varied variety of users, automobiles, equipment, and industries is made possible through the use of dynamic network slicing technologies by network operators.

(4) Energy-Efficient Communication: 6G will meet and exceed a number of requirements, including the delivery of high-energy performance, most notably in the context of widespread IoT use and an eco-system of numerous minute sensors. Additionally, extending the battery life of smartphones is necessary, in accordance with the assumption that their skills and abilities to deal with complex multimedia signal processing improve exponentially as their power consumption increases [385]. Thus, low energy consumption and extended battery charge life length are two study areas in 6G that aim to address the daily recharging challenges faced by the majority of communication equipment and to meet communication objectives. As a result, $6 \mathrm{G}$ must elicit a complete plan for energy-efficient wireless communication. A basic goal of $6 \mathrm{G}$ communication is to operate battery-free whenever and wherever possible, with a target efficiency of 1 pico-joule per bit $[386,387]$. Apart from the benefits of high-power $\mathrm{THz}$ waves, $6 \mathrm{G}$ communication enables directed beam communication via MIMO antenna arrays, allowing devices to deliver power beams in a specific direction. This technology has the ability to provide sufficient energy to devices covered by the network.

6G trends, technologies, and applications state that the services will redefine those provided by the $5 \mathrm{G}$ by morphing the classic URLLC, eMBB, and MMTC and providing new services (see Figure 5). The following services will be introduced: 


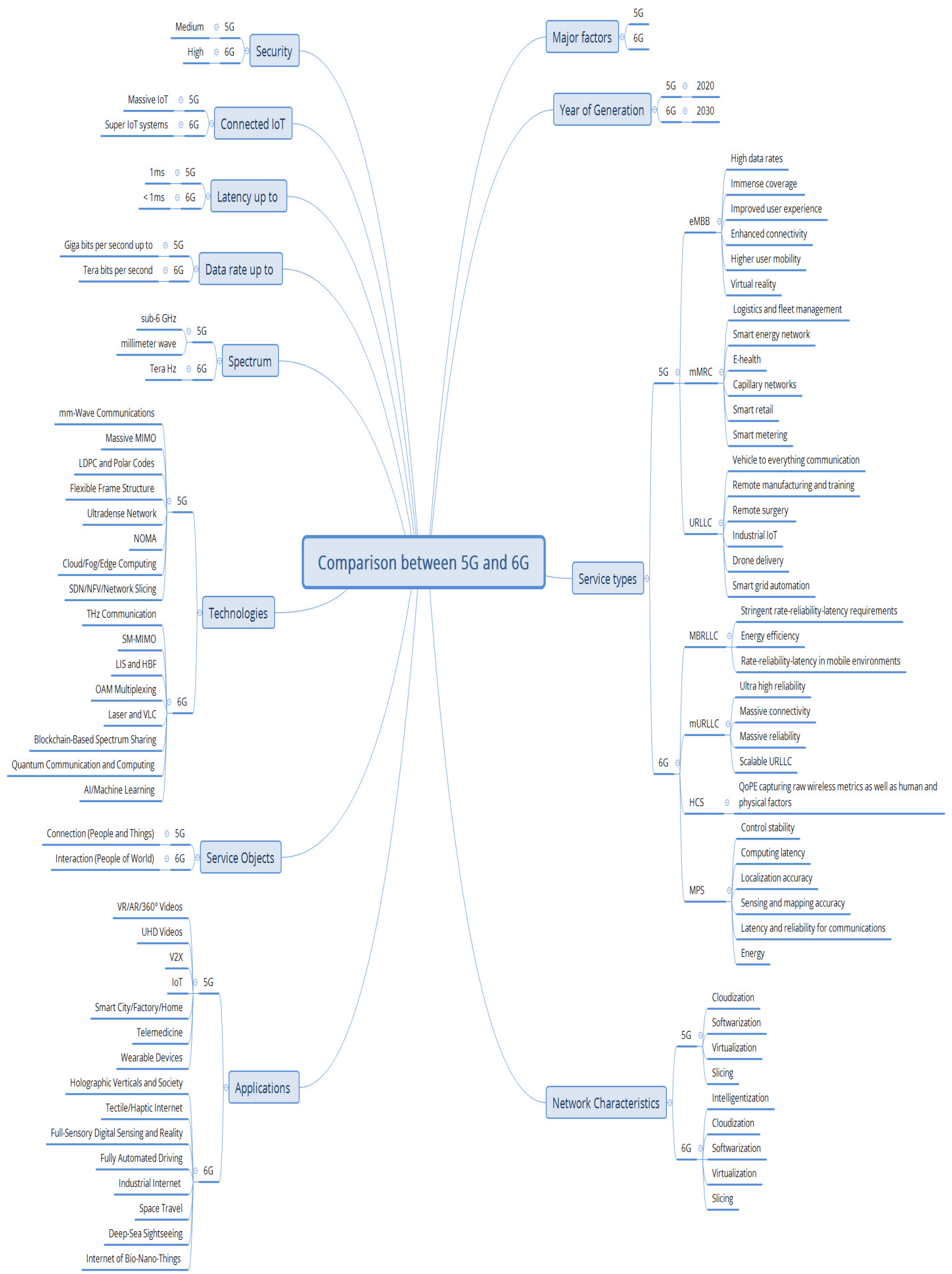

Figure 5. Comparison between 5G and 6G. Service Classes, Their Performance Indicators, And Example Applications. 


\section{Review and Survey Articles}

ML technologies are well described and summarised in survey and review papers based on the latest state of the art in 5G, 6G, and research documentation. The applications that utilise ML technologies are well described and summarised in survey and review articles. In this category, there are seventy-four articles, which have been sorted into five subcategories. In the following sections, we introduce the recent works in IoT, and energyefficiency in 5G, 6G as highlighted in Table 1 preceding to a discussion on open issues and challenges.

Table 1. Summary of the related review and survey papers.

\begin{tabular}{|c|c|c|c|c|c|c|c|c|}
\hline Ref. & Summary & $\begin{array}{l}\text { Machine } \\
\text { Leaning }\end{array}$ & & IoT & $\begin{array}{l}\text { Security } \\
\text { Approaches }\end{array}$ & VR \& AR & Energy & $\begin{array}{l}\text { Network } \\
\text { Architectures }\end{array}$ \\
\hline & & Deep Learning & $\begin{array}{l}\text { Other } \\
\text { Methods }\end{array}$ & ML & & & & \\
\hline [5] & $\begin{array}{l}\text { Made it more robust against var- } \\
\text { ious attacks. }\end{array}$ & & & $\checkmark$ & $\checkmark$ & & & \\
\hline [6] & $\begin{array}{l}\text { Workload-based EDoS and } \\
\text { Instantiation-based. }\end{array}$ & & $\checkmark$ & $\checkmark$ & & & & \\
\hline [7] & $\begin{array}{l}\text { Threats and sketches to mitigate } \\
\text { the security concerns. }\end{array}$ & & & $\checkmark$ & $\checkmark$ & & & $\checkmark$ \\
\hline [15] & $\begin{array}{l}\text { Virtualization explicitly de- } \\
\text { signed for IoT networks. }\end{array}$ & & $\checkmark$ & $\checkmark$ & & $\checkmark$ & & $\checkmark$ \\
\hline [16] & $\begin{array}{l}\text { 5G and Real-Time Communica- } \\
\text { tions. }\end{array}$ & & $\checkmark$ & $\checkmark$ & & $\checkmark$ & & $\checkmark$ \\
\hline [17] & $\begin{array}{l}\text { Various virtual and } A R \text { for the } \\
\text { world first } 5 \mathrm{G} \text {. }\end{array}$ & & $\checkmark$ & & & $\checkmark$ & & \\
\hline [18] & Fault management techniques. & & $\checkmark$ & & & $\checkmark$ & & $\checkmark$ \\
\hline [19] & Basic concepts of SON. & $\checkmark$ & & & & & $\checkmark$ & $\checkmark$ \\
\hline [20] & $\begin{array}{l}\text { Self-organizing networks solu- } \\
\text { tions. }\end{array}$ & $\checkmark$ & & $\checkmark$ & & & $\checkmark$ & $\checkmark$ \\
\hline [21] & $\begin{array}{l}\text { Application areas of AI/ML in } \\
\text { SDN and NFV based networks. }\end{array}$ & $\checkmark$ & & & $\checkmark$ & & & $\checkmark$ \\
\hline [22] & $\begin{array}{l}\text { Use cases and scenarios of 5G in } \\
\text { which ML. }\end{array}$ & & $\checkmark$ & & $\checkmark$ & & $\checkmark$ & $\checkmark$ \\
\hline [23] & $\begin{array}{l}\text { Challenges from these } 5 \mathrm{G} \text { fea- } \\
\text { tures and present several techno- } \\
\text { logical. }\end{array}$ & & $\checkmark$ & $\checkmark$ & & & & $\checkmark$ \\
\hline [24] & $\begin{array}{l}\text { DRL to address the RA prob- } \\
\text { lems. }\end{array}$ & & $\checkmark$ & & & & $\checkmark$ & $\checkmark$ \\
\hline [25] & $\begin{array}{l}\text { DRL based resource manage- } \\
\text { ment schemes for } 5 \mathrm{G} \text { HetNets. }\end{array}$ & A & & & & & $\checkmark$ & $\checkmark$ \\
\hline [26] & $\begin{array}{l}\text { Big data analytics solution for } 5 \mathrm{G} \\
\text { network. }\end{array}$ & $\checkmark$ & & & & & & $\checkmark$ \\
\hline [27] & $\begin{array}{l}\text { ML to assist channel modelling } \\
\text { and estimation has been intro- } \\
\text { duced. }\end{array}$ & $\checkmark$ & & & & & $\checkmark$ & $\checkmark$ \\
\hline [28] & AI and ML for 5G network. & & $\checkmark$ & & & & $\checkmark$ & $\checkmark$ \\
\hline [29] & $\begin{array}{l}\text { 5G network management, which } \\
\text { takes an end-to-end view of the } \\
\text { network. }\end{array}$ & & $\checkmark$ & & $\checkmark$ & $\checkmark$ & $\checkmark$ & $\checkmark$ \\
\hline [30] & $\begin{array}{l}\text { Improve the efficiency, latency, } \\
\text { and reliability of the current and } \\
\text { real-time network applications. }\end{array}$ & $\checkmark$ & & & & & $\checkmark$ & $\checkmark$ \\
\hline [31] & QoS for Users in 5G. & $\checkmark$ & & & & & $\checkmark$ & $\checkmark$ \\
\hline [32] & $\begin{array}{l}\text { Capabilities of DL systems to ex- } \\
\text { plain to network operators are } \\
\text { described below. }\end{array}$ & & $\checkmark$ & & & & $\checkmark$ & $\checkmark$ \\
\hline [33] & $\begin{array}{l}\text { Capabilities of DL systems to ex- } \\
\text { plain and justify their sugges- } \\
\text { tions to network operators. }\end{array}$ & $\checkmark$ & & $\checkmark$ & & & $\checkmark$ & $\checkmark$ \\
\hline [34] & Slice Network. & & $\checkmark$ & & & $\checkmark$ & & $\checkmark$ \\
\hline
\end{tabular}


Table 1. Cont.

\begin{tabular}{|c|c|c|c|c|c|c|c|c|}
\hline Ref. & Summary & $\begin{array}{l}\text { Machine } \\
\text { Leaning }\end{array}$ & & IoT & $\begin{array}{l}\text { Security } \\
\text { Approaches }\end{array}$ & VR \& AR & Energy & $\begin{array}{l}\text { Network } \\
\text { Architectures }\end{array}$ \\
\hline & & Deep Learning & $\begin{array}{l}\text { Other } \\
\text { Methods }\end{array}$ & ML & & & & \\
\hline [35] & $\begin{array}{l}\text { Detection principles that are op- } \\
\text { timal or near-optimal for huge } \\
\text { MIMO systems. }\end{array}$ & $\checkmark$ & & & & $\checkmark$ & $\checkmark$ & $\checkmark$ \\
\hline$[37]$ & $\begin{array}{l}\text { Outlined the current 5G testbed } \\
\text { in Prato. }\end{array}$ & & & $\checkmark$ & $\checkmark$ & & $\checkmark$ & $\checkmark$ \\
\hline [38] & $\begin{array}{l}\text { Effectively } \quad \text { integrating } \\
\text { SDN/NFV. }\end{array}$ & & $\checkmark$ & & & & & $\checkmark$ \\
\hline [39] & $\begin{array}{l}\text { AI for } 5 G \text { wireless communica- } \\
\text { tion systems. }\end{array}$ & A & & $\checkmark$ & $\checkmark$ & & $\checkmark$ & $\checkmark$ \\
\hline [40] & $\begin{array}{l}\text { ML in conjunction with proxim- } \\
\text { ity to real-time information tech- } \\
\text { nology solutions. }\end{array}$ & $\checkmark$ & & $\checkmark$ & & & $\checkmark$ & $\checkmark$ \\
\hline$[41]$ & $\begin{array}{l}\text { Investigates the potential fea- } \\
\text { tures of B5G. }\end{array}$ & $\checkmark$ & & $\checkmark$ & $\checkmark$ & $\checkmark$ & $\checkmark$ & $\checkmark$ \\
\hline [42] & $\begin{array}{l}\text { Mobile and wireless networking } \\
\text { research based on DL. }\end{array}$ & $\checkmark$ & & $\checkmark$ & $\checkmark$ & $\checkmark$ & $\checkmark$ & $\checkmark$ \\
\hline [52] & $\begin{array}{l}\text { To enhance the productivity of } \\
\text { the system and to avoid the de- } \\
\text { lays. }\end{array}$ & & & $\checkmark$ & $\checkmark$ & $\checkmark$ & $\checkmark$ & \\
\hline [53] & $\begin{array}{l}\text { Inductive analysis of the technol- } \\
\text { ogy required for driverless driv- } \\
\text { ing and } 5 G \text { technology. }\end{array}$ & & $\checkmark$ & $\checkmark$ & $\checkmark$ & & & \\
\hline [54] & $\begin{array}{l}\text { Explains FL ideas with an em- } \\
\text { phasis on their implementation } \\
\text { in automotive networks. }\end{array}$ & & $\checkmark$ & $\checkmark$ & $\checkmark$ & & $\checkmark$ & \\
\hline [55] & $\begin{array}{l}\text { Control may be delegated to clus- } \\
\text { ters of IoT nodes beyond the } \\
\text { edge gateway. }\end{array}$ & & & $\checkmark$ & & & $\checkmark$ & \\
\hline [56] & $\begin{array}{l}\text { Offered network is the founda- } \\
\text { tion of the semantics of a techno- } \\
\text { logical level of egovernment. }\end{array}$ & & $\checkmark$ & $\checkmark$ & $\checkmark$ & & $\checkmark$ & \\
\hline [57] & IoT systems and services. & & $\checkmark$ & $\checkmark$ & $\checkmark$ & $\checkmark$ & & \\
\hline [58] & Financial related issues. & & & $\checkmark$ & & & $\checkmark$ & \\
\hline [59] & $\begin{array}{l}\text { View existing wireless IoT con- } \\
\text { nectivity technologies can be ef- } \\
\text { fectively used to enable massive } \\
\text { connectivity for IoT. }\end{array}$ & & $\checkmark$ & $\checkmark$ & $\checkmark$ & $\checkmark$ & & \\
\hline$[60]$ & $\begin{array}{l}\text { ML approaches to UAV-based } \\
\text { communications has the poten- } \\
\text { tial to improve a variety of de- } \\
\text { sign aspects. }\end{array}$ & $\checkmark$ & & $\checkmark$ & $\checkmark$ & & $\checkmark$ & \\
\hline$[61]$ & $\begin{array}{l}\text { IoT devices are designed to trans- } \\
\text { mit little data packets with good } \\
\text { energy efficiency. }\end{array}$ & & & $\checkmark$ & $\checkmark$ & $\checkmark$ & $\checkmark$ & \\
\hline$[62]$ & $\begin{array}{l}\text { ITSs cyber-security, energy- } \\
\text { efficient utilization of SGs, } \\
\text { effective use of UAVs to assure } \\
\text { the best services of 5G. }\end{array}$ & $\checkmark$ & & $\checkmark$ & $\checkmark$ & & $\checkmark$ & \\
\hline$[63]$ & $\begin{array}{l}\text { 5G technologies are listed and de- } \\
\text { scribed how these features effect } \\
\text { the industries of the future. }\end{array}$ & & $\checkmark$ & $\checkmark$ & $\checkmark$ & $\checkmark$ & $\checkmark$ & \\
\hline [64] & $\begin{array}{l}\text { Investigate the } 5 \mathrm{G} \text { usability in } \\
\text { disasters. }\end{array}$ & & & $\checkmark$ & $\checkmark$ & $\checkmark$ & & \\
\hline$[65]$ & $\begin{array}{l}\text { Construction of a semantic net- } \\
\text { work comprising the most recent } \\
\text { concepts in the information. }\end{array}$ & & $\checkmark$ & $\checkmark$ & $\checkmark$ & $\checkmark$ & & \\
\hline$[67]$ & $\begin{array}{l}\text { IoT technology is examined from } \\
\text { a high-level perspective. }\end{array}$ & $\checkmark$ & & $\checkmark$ & $\checkmark$ & $\checkmark$ & $\checkmark$ & \\
\hline [68] & $\begin{array}{l}\text { Application of a low-complexity } \\
\text { Qlearning approach in the } \\
\text { mMTC situation. }\end{array}$ & $\checkmark$ & & $\checkmark$ & $\checkmark$ & $\checkmark$ & $\checkmark$ & \\
\hline
\end{tabular}


Table 1. Cont.

\begin{tabular}{|c|c|c|c|c|c|c|c|c|}
\hline Ref. & Summary & $\begin{array}{l}\text { Machine } \\
\text { Leaning }\end{array}$ & & IoT & $\begin{array}{l}\text { Security } \\
\text { Approaches }\end{array}$ & VR \& AR & Energy & $\begin{array}{l}\text { Network } \\
\text { Architectures }\end{array}$ \\
\hline & & Deep Learning & $\begin{array}{l}\text { Other } \\
\text { Methods }\end{array}$ & ML & & & & \\
\hline [71] & $\begin{array}{l}\text { Energy efficiency of radio access, } \\
\text { which is at the heart of wireless } \\
\text { networks. }\end{array}$ & & $\checkmark$ & & & $\checkmark$ & $\checkmark$ & \\
\hline [73] & $\begin{array}{l}\text { Big data and the promise of ML } \\
\text { for optimization and decision- } \\
\text { making in } 5 \mathrm{G} \text { networks. }\end{array}$ & $\checkmark$ & & $\checkmark$ & $\checkmark$ & $\checkmark$ & $\checkmark$ & \\
\hline [74] & $\begin{array}{l}\text { Classified these three pillars, soft- } \\
\text { warization, energy harvesting, } \\
\text { and optimization. }\end{array}$ & $\checkmark$ & & & & $\checkmark$ & $\checkmark$ & \\
\hline [8] & $\begin{array}{l}\text { AI into } 6 \mathrm{G} \text { and state of the art in } \\
\text { ML. }\end{array}$ & & $\checkmark$ & $\checkmark$ & $\checkmark$ & & $\checkmark$ & \\
\hline [9] & ML and privacy in $6 \mathrm{G}$ & $\checkmark$ & $\checkmark$ & $\checkmark$ & $\checkmark$ & & $\checkmark$ & \\
\hline [10] & $\begin{array}{l}\text { AI integrated into localization, } \\
\text { UAV communication, surveil- } \\
\text { lance, security and privacy } \\
\text { preservation. }\end{array}$ & $\checkmark$ & & $\checkmark$ & $\checkmark$ & & $\checkmark$ & \\
\hline [12] & $\begin{array}{l}\text { Addressing the shortcomings } \\
\text { and limitations of IoT and } \\
\text { Blockchain. }\end{array}$ & & $\checkmark$ & $\checkmark$ & & & $\checkmark$ & $\checkmark$ \\
\hline [13] & $\begin{array}{l}\text { Dimensions of a } 6 \mathrm{G} \text { network, in- } \\
\text { cluding the air interface. }\end{array}$ & & $\checkmark$ & $\checkmark$ & $\checkmark$ & & $\checkmark$ & \\
\hline [43] & $\begin{array}{l}\text { Requirements and challenges of } \\
\text { 6G. }\end{array}$ & & $\checkmark$ & $\checkmark$ & $\checkmark$ & & $\checkmark$ & \\
\hline [44] & $\begin{array}{l}\text { Added features and key perfor- } \\
\text { mance indicators of } 5 G \text { NR. }\end{array}$ & $\checkmark$ & & $\checkmark$ & $\checkmark$ & & $\checkmark$ & $\checkmark$ \\
\hline [45] & $\begin{array}{l}\text { Highlighting energy efficiency } \\
\text { spectral efficiency security, se- } \\
\text { crecy, and privacy adorability } \\
\text { and customization. }\end{array}$ & $\checkmark$ & & $\checkmark$ & $\checkmark$ & & $\checkmark$ & \\
\hline [46] & $\begin{array}{l}\mathrm{THz} \text { band antenna, fabrication } \\
\text { and measurement are presented. }\end{array}$ & & $\checkmark$ & & $\checkmark$ & & & $\checkmark$ \\
\hline [47] & $\begin{array}{l}\text { Blockchain-Envisioned UAV } \\
\text { Communication. }\end{array}$ & & $\checkmark$ & $\checkmark$ & $\checkmark$ & & $\checkmark$ & \\
\hline [66] & $\begin{array}{l}6 \mathrm{G} \text { to IoT technologies and ser- } \\
\text { vice areas. }\end{array}$ & $\mathrm{A}$ & & $\checkmark$ & $\checkmark$ & & $\checkmark$ & \\
\hline [75] & Vision and requirements of 6G. & $\checkmark$ & & $\checkmark$ & $\checkmark$ & & $\checkmark$ & \\
\hline [11] & $\begin{array}{l}\text { Investigations security and pri- } \\
\text { vacy problems associated with } \\
6 \mathrm{G} \text { technologies. }\end{array}$ & & $\checkmark$ & & $\checkmark$ & & $\checkmark$ & $\checkmark$ \\
\hline [48] & $\begin{array}{l}\text { Application of learning, predic- } \\
\text { tion, and decision-making to } \\
\text { manage the stream of humans. }\end{array}$ & $\checkmark$ & & $\checkmark$ & $\checkmark$ & & $\checkmark$ & \\
\hline [72] & $\begin{array}{l}\text { Analyze hardware reuse and } \\
\text { multiplexing solution to ease the } \\
\text { design of UEs that are both cost- } \\
\text { effective and energy-efficient. }\end{array}$ & $\checkmark$ & & $\checkmark$ & $\checkmark$ & & $\checkmark$ & $\checkmark$ \\
\hline [77] & $\begin{array}{l}\text { Ability to triple the adaptivity of } \\
\text { mmWave and THz. }\end{array}$ & & $\checkmark$ & $\checkmark$ & & & $\checkmark$ & $\checkmark$ \\
\hline [76] & $\begin{array}{l}\text { Vision for machine type commu- } \\
\text { nication in } 6 \mathrm{G} \text {. }\end{array}$ & & $\checkmark$ & $\checkmark$ & $\checkmark$ & & $\checkmark$ & \\
\hline [69] & $\begin{array}{l}\text { Vision for machine type commu- } \\
\text { nication in } 6 \mathrm{G} \text {. }\end{array}$ & & $\checkmark$ & $\checkmark$ & $\checkmark$ & & $\checkmark$ & \\
\hline [70] & $\begin{array}{l}\text { ML, QC, and QML identify their } \\
\text { potential benefits, issues in the } \\
\text { B5G networks. }\end{array}$ & $\checkmark$ & $\checkmark$ & $\checkmark$ & $\checkmark$ & & & $\checkmark$ \\
\hline [49] & $\begin{array}{l}\text { 6G challenges, requirements, } \\
\text { and trends. }\end{array}$ & & $\checkmark$ & $\checkmark$ & $\checkmark$ & & & $\checkmark$ \\
\hline [78] & $\begin{array}{l}\text { Applying TL to future } 6 \mathrm{G} \text { com- } \\
\text { munications. }\end{array}$ & & $\checkmark$ & $\checkmark$ & & & $\checkmark$ & $\checkmark$ \\
\hline [51] & $\begin{array}{l}\text { Evolution of KPIs and technol- } \\
\text { ogy trends towards } 6 \mathrm{G} \text {. }\end{array}$ & & & & $\checkmark$ & & & $\checkmark$ \\
\hline
\end{tabular}


Table 1. Cont.

\begin{tabular}{|c|c|c|c|c|c|c|c|c|}
\hline Ref. & Summary & $\begin{array}{l}\text { Machine } \\
\text { Leaning }\end{array}$ & & IoT & $\begin{array}{l}\text { Security } \\
\text { Approaches }\end{array}$ & VR \& AR & Energy & $\begin{array}{l}\text { Network } \\
\text { Architectures }\end{array}$ \\
\hline & & Deep Learning & $\begin{array}{l}\text { Other } \\
\text { Methods }\end{array}$ & ML & & & & \\
\hline [14] & $\begin{array}{l}\text { Explore the role of blockchain to } \\
\text { address formidable challenges in } \\
6 \mathrm{G} \text {. }\end{array}$ & & $\checkmark$ & $\checkmark$ & $\checkmark$ & & & $\checkmark$ \\
\hline [50] & $\begin{array}{l}\text { Potential challenge and propose } \\
\text { possible technical components in } \\
\text { a future } 6 \mathrm{G} \text { system. }\end{array}$ & & $\checkmark$ & $\checkmark$ & & & $\checkmark$ & $\checkmark$ \\
\hline [36] & $\begin{array}{l}\text { Deals with the answers to those } \\
\text { questions for the health vertical } \\
\text { of } 6 \mathrm{G} \text {. }\end{array}$ & & $\checkmark$ & $\checkmark$ & & & $\checkmark$ & $\checkmark$ \\
\hline
\end{tabular}

The first group—security approaches The review and survey articles look into the framework or platform model that will be developed based on the security approaches. Only one article [5] dissected attacks such as call fraud and data interception in great detail. Several strategies, such as data encryption, mutual authentication, and other approaches can be used to address this issue. A study published in the article [6] examined the EDoS problem in emerging network situations. SON and NFV are the foundations for adaptive approaches. The author provided an overview of how multiple fog devices communicate with one another using Internet of Things paradigms. Describes these dangers and lays out future research on how to minimise the security concerns raised by a modern distributed control infrastructure [7].

Two articles [8,9] comprehensively examine the use of machine learning approaches to significant 6G network concerns such as enhanced radio interface, intelligent traffic control, security protection, administration and orchestration, and network optimization. Furthermore, the latest status of important standardisation initiatives and industry research programmes on applying machine learning to mobile networks transitioning to $6 \mathrm{G}$ is examined. Only article [10] provided a full overview of AI-enabled 6G communication technology, which has a wide range of potential uses in the future. Examine how AI can be used in a variety of applications, including object localisation, UAV communication, surveillance, security, and privacy protection, among others.

Finally, a use case was addressed that demonstrates the employment of AI principles in an intelligent transportation system. Only one article [11] was written about the current state of 6G security and privacy. The survey starts with a look back at earlier networking technologies and how they influenced contemporary 6G networking trends. Then, we'll go through four major components of $6 \mathrm{G}$ networks: real-time intelligent edge computing, distributed artificial intelligence, intelligent radio, and 3D intercoms, as well as some interesting upcoming technologies in each area, as well as the security and privacy challenges that come with them.

Three papers [12-14] examined the major obstacles in integrating Blockchain and IoT technologies in order to achieve high-level solutions by addressing the weaknesses and limits of both technologies. Many IoT concerns can be addressed with blockchain, however, any merger of two embedded technologies brings additional issues and impediments.

Second group: virtual and augmented reality virtualization Examine 5G and 6G approaches and categorise them into various types of solutions.

Two studies $[15,16]$ reviewed virtualization solutions specifically developed for IoT networks, allowing users and operators to create environments that match their demands while coexisting with other networks in the same physical space.The article [17] looks at the characteristics of the fourth industrial revolution and considers 5G's potential as a GPT. Other researchers offered a new classification of recent fault management research accomplishments in network virtualization environments, as well as a comparison of their primary contributions and flaws [18]. 
Third group, network architectures Several architectures have been presented by various research units in order to better integrate these services. The first section of this article provides an overview of the connected services managed by the $5 \mathrm{G}$ and $6 \mathrm{G}$ networks. After that, we'll talk about existing architectures. The essential concepts of selforganizing networks were reviewed and explored in four publications [19-22]. In addition, provide a taxonomy for self-organizing networks use cases and describe their underlying ML techniques, as well as service management by demonstrating how combining ML algorithms with SDN and NFV on a variety of use cases and scenarios can yield insights, detect meaningful events and conditions, and enable the management system to respond appropriately. NMA was discussed in one publication [23] as a way to improve the level of intelligence in network elements. Two papers $[24,25]$ investigated resource allocation in 5G communications utilising RL, as well as a dynamic programming framework for solving RA problems optimally across different network states.

Three publications [26-28] examined big data in the context of 5G and machine learning. The vast data can be divided into two categories: raw data and correct data. If the right data is extracted efficiently from such a large amount of raw data, $5 \mathrm{G}$ can be optimised. Big data analytics has evaluated the main taxonomy of machine learning and the fresh trends that could enable this exploitation of data to get network insight a reality. One study [29] examined open data choices as well as alternate ways to obtain data from networks that would otherwise be unavailable to academics. On the IoT side, data storage and synchronisation, cloud storage, the base control system, third-party apps, and the management interface are all available.

Two articles [30,31] introduced the potential of $\mathrm{AI}$ in the next-generation wireless networks, ranging from basic learning algorithms such as ML, DL, and others, to help meet the varying requirements of the $5 \mathrm{G}$ standards, such as operating in a fully automated manner, meeting increased capacity demand, and providing superior QoE to users.

Only one article [32] looked at the application of machine learning to fault management in cellular networks from an operational standpoint. Based on the building components of a typical fault management system, describe the applicable ML approaches through to DL, and assess the progress that has been made in their implementation. The goal is to combine $5 \mathrm{G}$ technology with machine learning to make these technologies more accessible to a wider variety of consumers.

Three articles [33-36] used an empirical analysis of resource management efficiency in network slicing to investigate this trade-off. The results are based on extensive measurement data acquired in a live mobile network and provide insight into the efficiency of network slicing architectures, their dimensioning, and their interaction with resource management algorithms.

By presenting several case examples, discussing the problems, and shedding fresh light on future research paths for using AI. in 5G wireless communications, four articles [37-40] offered an in-depth assessment of AI. and ML for 5G wireless communication systems and networking. One publication [41] looked into the potential features of B5G, as well as future research directions for how machine learning might help realise B5G. Others covered a variety of strategies and platforms that make it easier to install DL on mobile devices. Then, using DL to organise mobile and wireless networking research into different categories, present an encyclopaedic review of the field [42].

Three articles [43-45] presented a systematic overview of 6G that focuses on prospects and development, core techniques, applicable scenarios, and challenges, and proposes a framework for the $6 \mathrm{G}$ network, as well as an overview of the first five generations of wireless systems, followed by a survey on the $6 \mathrm{G}$ wireless network, as well as a discussion on the possible requirements and challenges.

Specifications for 6G antennas for various applications are highlighted in [46]. A comprehensive review of recent $\mathrm{THz}$ band antenna manufacturing and measurement work is provided. The design, construction, and measurement of $\mathrm{THz}$ band antennas are discussed. $\mathrm{THz}$ band antenna research directions for 6G technology are addressed, as well as $\mathrm{THz}$ 
band antenna design, fabrication, and testing. A description of future research prospects for the integration of blockchain and 6G technologies in UAV communications is offered in another work [47]. Then, show a case study of a blockchain-based UAV communication system that uses $6 \mathrm{G}$ networks to secure Industry 4.0 applications.

Another study [48] discusses how integrating AI in URLLC improves multi-level architecture and provides a technique for constructing wireless networks. This is accomplished by using learning, prediction, and decision-making to manage the large number of people who have been trained by big data. The research paper's secondary goal is to improve a multi-level design.

Two publications $[49,50]$ looked into the issues, requirements, and trends around 6G. Also, talk about how AI approaches can help with 6G. Identify some new exciting $6 \mathrm{G}$ services and use-cases based on the requirements and solutions that $5 \mathrm{G}$ cannot adequately serve.

A study on the evolution of KPIs and technical trends toward 6G was published in one publication [51]. Short-term (2022'ish), medium-term (2025'ish), and long-term (2030'ish) timelines are all considered. Forecasts on developing use cases and their requirements, as well as assumptions on the pace of wireless technology improvements, are used to examine the evolution of these KPIs.

The fourth group 5G, 6G-IoT Information of Things, is discussed as well as in regard to the idea of merging IoT, 5G, and 6G.

Three papers [52-54] reported on the evolving automotive cyber security, which can use $5 \mathrm{G}$ to be faster and more efficient. Presented a peer-to-peer IoT infrastructure, built to OpenFog standards, which moves control out to "things" without involving devices or cloud computing. A new article [55] offered a 'Disruptive Networking' strategy, which enables many IoT nodes to take part in autonomous and cooperative decision-making. Other reports [56] explore methods of transitioning the healthcare and renewable energy industries from e-government to smart government.

Three papers [57-59] examined IoT-based strategies for the 5G mobile communications transition. Complex IoT systems necessitate heterogeneous computing and high-speed communication networks like 5G. Several real-world IoT systems infrastructures utilising heterogeneous computing are also introduced. Classify and review different wireless IoT connectivity solutions, starting with the connectivity range.

Four papers [60-63] presented practical approaches for the smooth integration of smart city applications in 5G networks. In-depth use of ITSs, cyber-security, energy-efficient use of SGs, effective use of UAVs for the provision of the best $5 \mathrm{G}$ and B5G services, and a smart health care system in a smart city.

Three [64-66] studies evaluated how 5G technology could help with the management of disasters. The way to managing government, corporate, and personal disaster responses is to use Big Data, IoT, social media, and cloud computing.

Two different publications $[67,68]$ summarise the main enabling technologies as well as a whole slew of new developing uses of 5G-IoT, each tied to advancements in artificial intelligence, $\mathrm{ML}$, and digital edge computing. This post outlined the essential enablers for mMTC in cellular networks.

One article [69] article investigated different issues associated with fog computing in 6G-enabled large IoT. Categorize several energy-efficient fog computing options for IoT and summarise the current progress. Future projects and open potential to build energy-efficient fog computing solutions are discussed at last. One study [70] provides a full analysis of ML, QC, and QML, highlighting their possible benefits, challenges, and use cases for the B5G networks. A QC-assisted and QML-based framework for 6G communication networks was proposed, where it outlined the infrastructure, edge, air interface, and user end.

Final Group Energy Efficiency of 5G and 6G 5G and 6G analyses the most recent works that address energy efficiency. We offer an overview of 5G, 6G, enormous IoT, and it concentrates on recent works. 
Two articles summarise current work on the radio access and core of wireless networks and identify ongoing difficulties and opportunities. The following are paths for power optimization: using game theory and machine learning, as well as different energy-saving measures [71,72].

This paper proposed that future cellular networks will have different system requirements, which include utilising energy-harvesting technologies and optimising tool sets [73].

Another article discusses the opportunities and problems given by 5G networks. This review discusses the latest progress on standardisation, possible architecture candidates, and the energy considerations in 5G networks [74]. The authors [75,76] presented a panoramic perspective of the supporting technologies for 6G and 6G-capable applications, such as multi-sensory-extended reality, digital replica, and more. There are over ten new applications of the $6 \mathrm{G}$ technology that are only just starting to be implemented. Furthermore, explore the multi-faceted communication possibilities of $6 \mathrm{G}$, which will bring about a drastic transformation in the corporate arena.

In next-generation applications of mmWave and $\mathrm{THz}$ solutions, space-air-ground integrated networks, full-duplex techniques, and other sophisticated channel coding assisted system designs, where powerful machine learning algorithms are expected to make autonomous decisions about the best mode of operation with minimal human intervention, this intelligent Tripple-fold adaptivity offers significant benefits [77].

One article [78] described, as mentioned above, requirements were contradictory, such as high efficiency and high density. This combination eventually led to reduced energy efficiency. TL can alleviate practically every problem caused by $6 \mathrm{G}$ needs.

\section{Overview of Existing Technique}

A scheme, structure type, or technique model is investigated in this second section, which contains 61 papers, to meet the requirements of the stage where the results of the research on ML are to be addressed, as well as the methodology used by 5G and 6G for the Internet of Things and energy transmissions. The following Table 2 preceding to a discussion subsections provide an overview of six major categories, including network resource management, security, augmented reality, network scaling, resource allocation, and the smart grid.

Table 3 summarises the possible services, core idea, and solutions uses of Existing Technique related to machine learning in 5G and 6G-based energy technologies.

Table 2. Overview of Existing Technique.

\begin{tabular}{|c|c|c|c|c|c|}
\hline Ref. & Technique & Core Term & Core Idea & Solution & Drawbacks \\
\hline $1-$ & Network & Resource & Management & & \\
\hline [79] & URLLC. & $\begin{array}{l}\text { Functions } \\
\text { needed for } \\
\text { defining and } \\
\text { automating } \\
5 G \text { experi- } \\
\text { ments. }\end{array}$ & $\begin{array}{l}\text { E2E configuration of } 5 \mathrm{G} \\
\text { testbeds is supervised } \\
\text { throughout. }\end{array}$ & $\begin{array}{l}\text { Framework deployment, con- } \\
\text { trol, management, monitor- } \\
\text { ing, analytics, and security in } \\
5 \mathrm{G} \text { testbeds. }\end{array}$ & $\begin{array}{l}\text { Vertical should select a test case } \\
\text { from a list of test cases for a sep- } \\
\text { arate KPI. }\end{array}$ \\
\hline [80] & OpenAirInterface. & Performance. & $\begin{array}{l}\text { OAI is essential to the devel- } \\
\text { opment of the key } 5 \mathrm{G} \text { tech- } \\
\text { nologies. }\end{array}$ & $\begin{array}{l}\text { 3GPP-compliant LTE sys- } \\
\text { tems for real-time in- } \\
\text { door/outdoor testing and } \\
\text { demonstration. }\end{array}$ & $\begin{array}{l}\text { Demands adaptable and realistic } \\
\text { experimentation platforms that } \\
\text { support a wide range of experi- } \\
\text { mentation modalities. }\end{array}$ \\
\hline [81] & STRAIGHT. & $\begin{array}{l}\text { Mobility state } \\
\text { estimation. }\end{array}$ & $\begin{array}{l}\text { UE mobility which complies } \\
\text { with UE historical informa- } \\
\text { tion standards. }\end{array}$ & $\begin{array}{l}\text { Compensate for varying mo- } \\
\text { bility classes, both low and } \\
\text { high. }\end{array}$ & $\begin{array}{l}\text { Complex networks with overlap- } \\
\text { ping cells of varied sizes. }\end{array}$ \\
\hline [82] & D2D mode. & $\begin{array}{l}\text { SINR proxim- } \\
\text { ity distance } \\
\text { and battery } \\
\text { consumption. }\end{array}$ & $\begin{array}{l}\text { Dynamic network's mode se- } \\
\text { lection method. }\end{array}$ & $\begin{array}{l}\text { Delivered a better outcome } \\
\text { than conventional mode se- } \\
\text { lection up to } 30 \% \text {. }\end{array}$ & $\begin{array}{l}\text { Mode selection applied at the } \\
\text { BS, and device-centric principles } \\
\text { should be employed to accom- } \\
\text { plish } 5 \text { G network goals. }\end{array}$ \\
\hline
\end{tabular}


Table 2. Cont.

\begin{tabular}{|c|c|c|c|c|c|}
\hline Ref. & Technique & Core Term & Core Idea & Solution & Drawbacks \\
\hline [83] & CMAC. & $\begin{array}{l}\text { Cooperative } \\
\text { caching. }\end{array}$ & $\begin{array}{l}\text { CMAC method to lower the } \\
\text { average delay of providing } \\
\text { material. }\end{array}$ & $\begin{array}{l}\text { Attentively monitor QoE and } \\
\text { content-access latency. }\end{array}$ & $\begin{array}{l}\text { Various trace-driven simulations } \\
\text { show that CMAC offers up to } \\
13 \% \text { less average content-access } \\
\text { latency. }\end{array}$ \\
\hline [84] & DICE. & $\begin{array}{l}\text { Network protocol } \\
\text { design. }\end{array}$ & $\begin{array}{l}\text { The DICE ICN forwarding } \\
\text { strategy. }\end{array}$ & $\begin{array}{l}\text { Delivered up to } 2 X \text { more suc- } \\
\text { cessful delivery and incurs } \\
\text { just a tenth of the network } \\
\text { overhead. }\end{array}$ & $\begin{array}{l}\text { To optimise the energy consump- } \\
\text { tion of different wireless tech- } \\
\text { nologies. }\end{array}$ \\
\hline [85] & $\begin{array}{l}\text { Tactile Inter- } \\
\text { net. }\end{array}$ & $\begin{array}{l}\text { Network compo- } \\
\text { nents. }\end{array}$ & $\begin{array}{l}\text { Internet system using SDN } \\
\text { in the core of the cellular } \\
\text { network and MEC in multi- } \\
\text { levels. }\end{array}$ & $\begin{array}{l}\text { Even though } 1 \mathrm{~ms} \text { round-trip } \\
\text { latency can be a challenge, } \\
\text { Tactile Internet has proven to } \\
\text { be a success. }\end{array}$ & $\begin{array}{l}\text { To reduce the round-trip latency } \\
\text { is to limit the number of network } \\
\text { nodes engaged in the communi- } \\
\text { cation process. }\end{array}$ \\
\hline [86] & $\begin{array}{l}\text { Mobility Man- } \\
\text { agement. }\end{array}$ & $\begin{array}{l}\text { Transport proto- } \\
\text { cols. }\end{array}$ & $\begin{array}{l}\text { Performance evaluation of } \\
\text { TCP on mmWave cellular sys- } \\
\text { tems with mobility manage- } \\
\text { ment. }\end{array}$ & $\begin{array}{l}\text { Increasing network density } \\
\text { can greatly enhance the per- } \\
\text { formance of TCP with re- } \\
\text { spect to both throughput and } \\
\text { latency in mobile environ- } \\
\text { ments with blocking. }\end{array}$ & $\begin{array}{l}\text { Expand the simulation and put } \\
\text { it in the real world. Both } \\
\text { create network topologies that } \\
\text { lower the end-to-end latency of } \\
\text { a connection and implement a } \\
\text { performance-boosting proxy. }\end{array}$ \\
\hline [87] & eTOM. & QoS. & $\begin{array}{l}\text { Build a virtualized architec- } \\
\text { ture for dynamic delivery of } \\
\text { services, QoS, and increased } \\
\text { resource performance. }\end{array}$ & $\begin{array}{l}\text { All of these functionali- } \\
\text { ties were implemented } \\
\text { using modules within the } \\
\text { OpenStack cloud manager. }\end{array}$ & $\begin{array}{l}\text { To complete the OSR end-to-end } \\
\text { process grouping and the au- } \\
\text { tomation and support for FAB } \\
\text { framework functional blocks im- } \\
\text { plementation. }\end{array}$ \\
\hline
\end{tabular}

Wireless Spec- Network algo- oriented wireless spectrum

[88] trum Manage- Network algo- management technique, ment. rithms. based on cognitive radio technology. Cyber- $\quad$ OSM, a popular 5G manage-

[89] Physical Network services. $\begin{aligned} & \text { ment and orchestration plat- } \\ & \text { form was used to deploy vir- }\end{aligned}$ Systems. $\quad$ tual network functionalities.

NR access Numerology FDM Simulate $\begin{array}{lll}\text { NR access } & \text { QoS. } & \text { FDM numerology in the ns-3 } \\ \text { technology. }\end{array}$ network simulator.

Integration of

[91] Carrier Aggregation.

Network simulations.
Implementation for the mmWave ns-3 multiconnectivity techniques for 3GPP New Radio.
Before allocating resources, design a double pricing model that charges cognitive users lower fees.

Scenarios showing how $5 \mathrm{G}$ technologies might help the CPS sector.

Followed the 3GPP specifications to configure the time/frequency resource units automatically.

DC-implementation illustrated, along with details on the integration with CA.
A convenient mechanism, better suited to day-to-day scenarios.

Virtual services plus networking will be created by PNFs that can be made to the device quickly.

Puncturing the resources already committed for eMBB and designating processes to identify so would be essential.

To research additional CC management policies, which could benefit from a PHY-MAC crosslayer with additional channel information used in resource allocation.

Architecture mitigates latency and performance issues in essential network control and data gateways.

Integrate procedures for authentication and authorisation to build a scalable and secure solution in a $5 \mathrm{G}$ platform.

Open-source software ecosystem promotes concepts while sparking conversation on difficult practical elements of multi-administrative.

Mechanism seeks to let the cell transfer decisions assert their position at the SDN controller
Experimental results on orbit radio testbed latency and mobility. Integrate well-known procedures for authentication and authorisation to build a scalable and secure solution in a 5G platform.

Open conversations about speculative technology and ongoing research and development.

Decisions are made dynamic according to the changing conditions of the network. 
Table 2. Cont.

\begin{tabular}{|c|c|c|c|}
\hline Ref. & Technique & Core Term & Core Idea \\
\hline [96] & DIY model & Mobile networks. & $\begin{array}{l}\text { Constructing mobile net- } \\
\text { works in places where prior } \\
\text { community cellular net- } \\
\text { works were focused on low } \\
\text { end service provisioning. }\end{array}$ \\
\hline [97] & C-RAN. & $\begin{array}{lr}\text { Network } & \text { re- } \\
\text { sources } & \text { alloca- } \\
\text { tion. } & \end{array}$ & $\begin{array}{l}\text { Energy-efficient joint work- } \\
\text { load scheduling and BBU al- } \\
\text { location algorithm, utilising } \\
\text { queueing theory. }\end{array}$ \\
\hline [98] & AirSea. & Robotics. & $\begin{array}{l}\text { Clever manufacturing facility } \\
\text { in the sea. }\end{array}$ \\
\hline [99] & $\begin{array}{l}\text { Throughput } \\
5 \mathrm{G} \text {. }\end{array}$ & $\begin{array}{l}\text { Wireless access } \\
\text { networks. }\end{array}$ & $\begin{array}{l}\text { Offered a } 5 G \text { multi-cell ns-3 } \\
\text { simulation framework. }\end{array}$ \\
\hline [100] & $\begin{array}{l}\text { Augmenting } \\
\text { QoS. }\end{array}$ & QoS. & $\begin{array}{l}\text { V3I Cloud SDK toolchains } \\
\text { support creating complex au- } \\
\text { tomated cloud. }\end{array}$ \\
\hline [101] & $\begin{array}{l}\text { MEC in the } \\
\text { Cloud-RAN. }\end{array}$ & Core Network. & $\begin{array}{l}\text { Situation where disaggre- } \\
\text { gated base stations capable } \\
\text { of provisioning MEC capabil- } \\
\text { ities in per-packet. }\end{array}$ \\
\hline
\end{tabular}

[102] OpenAirInterfaceCloud-RAN.

Network func-

[103] tion virtualization.

Intent-based networking.
Modular SDN/NFV-based SON testbed for future 5G mobile networks.

Solution
$5 \mathrm{G}$ to spread to more ru-

ral and non-urban areas by empowering small-scale local operators and communities to create and run contemporary networks.

C-RAN controller distributes workload allocation among BBU servers in a time slot basis.

Sea-based, land-based, airbased, and space-based linkages are incorporated into the design of an air and sea manta ray robot.

$5 \mathrm{G}$ trace dataset, and a largescale multi-cell 5G/mmwave simulation framework.

Executed an experimental system in which automobiles formed a cloud resource unit.

Developed a signalling for communication between DUs and a MEC agent, which has access to container-based services.

A CDSA-based testbed is required to enable examination of the NG-SON capabilities for practical implementations.

Backhaul interface based on intent. Wireless controllers should have little contact with the wired backhaul controller. proach to mobile backhauling for $5 \mathrm{G}$ networks.

Investigated 5G mobile communication system performance, MAS with polarized antenna based BS system is applied.

Network sim- Network perforulations. mance analysis.

These principles were used [105] DES $\quad$ Maintenance per- to give real-world industrial formance.

$5 \mathrm{G}$ pilot deployment assessments.

Focus on very low latency and ultra-high reliability of

[106] FiWi.

FiWi access networks $5 \mathrm{G}$ and study how they can be obtained in FiWi upgraded LTE-A HetNets.

\section{G infrastructure emulator} capable of emulating a real-

[107] 5G infrastruc- Service Deployture emulator. ment.
Simulation results reveal that the misaligned polarisation causes more power loss for the polarised antenna structure.

Inputs will be used in the requirement definition for $5 \mathrm{G}$ networks, such as missioncritical clouds and analytics services, as well as other network services.

DOFR's proposed routing technique helped increase the aggregate FiWi enhanced LTE-A throughput substantially.

Proposed approach provides an emulator of $5 \mathrm{G}$ infrastructures, which can accurately replicate 5G infrastructure.

\section{Drawbacks}

Subscriber base can have recurring costs under $\$ 1$ USD per month.

For restricted power and cost budgets, the scheduling strategy is suitable.

Seaplane, WIG effect, and manta ray robots will significantly benefit ocean engineering and resource and energy development.

Incorporate mmwave and sub$6 \mathrm{GHz}$ as shown in actual-world next-generation $5 \mathrm{G}$ networks.

Under investigation and outcomes.

Test how service replication via edge nodes serves different base stations' technology mix.

Incorporate VNF migration, path restoration, and telemetry support for the Intent interface.

As a result, system capacity increases when the polarised antenna arrangement is implemented.

It is vital to use DES in tandem with establishing business justifications for investments.

Emulation platform and empirical data reported in terms of $5 \mathrm{G}$ service rollout times on bare metals. the-shelf servers via virtualization. 
Table 2. Cont.

\begin{tabular}{lllll}
\hline Ref. & Technique & Core Term & Core Idea & Solution \\
\hline $2-$ & Security & Approaches & & \\
\hline [108] & $\begin{array}{l}\text { Mobility man- } \\
\text { agement sys- } \\
\text { tem design. }\end{array}$ & $\begin{array}{l}\text { Virtualized to physical address } \\
\text { work. }\end{array}$ & $\begin{array}{l}\text { net- } \\
\text { encapsulation empowers mo- } \\
\text { bility capabilities, and mobil- } \\
\text { ity is implemented through a } \\
\text { flow table entry. }\end{array}$ & $\begin{array}{l}\text { indence rendered changes } \\
\text { in network availability unde- } \\
\text { tectable to the user. }\end{array}$ \\
\hline
\end{tabular}

[109] Data privacy. Communication Discussed the reasons why system security.
given in $3 \mathrm{G}$ or $4 \mathrm{G}$ networks.

\section{[110] 5GReasoner. Security models.}

[111] Bootstrapping Security and pri- techniques and threein Cellular vacy. Networks. Software- Software-Defined effecting the administration Defined Security. and orchestration of the virtuSecurity. Data Origin Authentica- Trust. tion.

Security Event Security and priManagement. vacy. [115] Privacy Network, architec- comparing the security of 5G ture.
Control-plane protocol protocols spanning across various layers of the $5 \mathrm{G}$ protocol stack.

Precomputation-based digital signature creation DPKI technique, protocol, and cryptographic scheme.

Proposed to integrate security into the slice life cycle, alization/softwarization architecture.

Studied the security protion in $5 \mathrm{G}$ networks.

To deliver security monitoring and correlation capabilities to mobile network operators, infrastructure service providers, and tenants, verticals, and horizontal applications.

Presented a detailed analysis wireless network systems to $4 \mathrm{G}$ cellular networks.

Models in the AKA family: 5G AKA. Extract 5G requirements from the 3GPP standards and discover missing security targets.

These might become adversaries against identity and location privacy.

Behaviour-specific abstrac tion enables an automated analysis of $5 \mathrm{G}$ Reasoner.

\section{Examine cryptography} backed authentication mechanisms to prevent adversaries from enticing unsuspecting cellular devices to connect to malicious base stations.

Security architecture is made up of built-in security features based on the ability to combine enforcement and monitoring operations within the software-defined network infrastructure.

Two attack scenarios were created to highlight an opponent that gains illegitimate data access via a stated source of authenticity.

Ensured automatic security operations and security services management using $5 \mathrm{G}$ network automated SLA.

Proposing a study on a security sharing method for $5 \mathrm{G}$

Utilize Tamarin to perform a complete security audit of the model with respect to the $5 \mathrm{G}$ security goals.

Proposed a mutual physical Designing a new handover authentication method for SDN aided 5G HetNets.
Drawbacks

Better control of heterogeneity and service scalability are provided by service decoupling

In both situations, it is the same security, but since it is for different networks, there are different reasons for offering the protection.

To integrate additional critical control-layer protocols.

To create a tailored cellular IoT scheme with 5G URLLC protocol.

The problem is in properly connecting network and computing resource control with network control.

Deferred signing class might be used to conserve computing resources but increasing communication costs.

Find strategies to control security in network slices. concentrate on metrics to estimate deployment and configuration impact on performance needs.

Security is not required, so saying that it is a disadvantage would not hinder the system in checking for it.

To see if AKA protocol versions such as $3 \mathrm{G}$ and $4 \mathrm{G}$ can deliver security benefits as compared to 5G AKA

Weighing parameters differ in simulations. Additionally, an evaluation is conducted using different SNRs and weighting parameters to examine proposed authentication technique. 
Table 2. Cont.

\begin{tabular}{|c|c|c|c|c|c|}
\hline Ref. & Technique & Core Term & Core Idea & Solution & Drawbacks \\
\hline [118] & WireGuard. & $\begin{array}{l}\text { Security and pri- } \\
\text { vacy. }\end{array}$ & $\begin{array}{l}\text { Alternative to IPsec, Wire- } \\
\text { Guard is proposed. Based } \\
\text { on the analysis, the influence } \\
\text { of security mechanisms on la- } \\
\text { tency is insignificant. }\end{array}$ & $\begin{array}{l}\text { Tested the eCPRI transporta- } \\
\text { tion in-depth. Even though } \\
\text { overhead from security pro- } \\
\text { tocols has minor impact on la- } \\
\text { tency, it is important to keep } \\
\text { reduced latency in mind. }\end{array}$ & $\begin{array}{l}\text { Interesting to use hardware to } \\
\text { minimise the latency of } 5 G \text { fron- } \\
\text { thaul. 5G can be maintained } \\
\text { without compromising security } \\
\text { by using a Quantum-secure ver- } \\
\text { sion of MACsec, IPsec, and Wire- } \\
\text { Guard. }\end{array}$ \\
\hline
\end{tabular}

\begin{tabular}{llll}
\hline 3- & Augmented & Reality & \\
\hline [119] & $\begin{array}{l}\text { Mobility man- } \\
\text { agement sys- } \\
\text { tem design. }\end{array}$ & $\begin{array}{l}\text { Virtualized } \\
\text { work. }\end{array}$ & $\begin{array}{l}\text { Virtual to physical address } \\
\text { encapsulation empowers mo- } \\
\text { bility capabilities, and mobil- } \\
\text { ity is implemented through a } \\
\text { flow table entry. }\end{array}$ \\
\hline
\end{tabular}

[120] 5G VR/AR. $\quad \begin{aligned} & \text { Human-centered } \\ & \text { computing. }\end{aligned}$

An effective representation method for constructing the 360 films is needed in order to avoid sending a huge 360 .

Investigate both dynamic and hybrid profiling, as well as adaptive partitioning, to address a demanding augmented reality scenario.

$\begin{array}{lll}\text { [121] AR applica- Network architec- } & \begin{array}{l}\text { and hybrid profiling, as well } \\ \text { as adaptive partitioning, } \\ \text { tion. }\end{array} & \begin{array}{l}\text { to address a demanding } \\ \text { augmented reality scenario. }\end{array}\end{array}$

[122] VR and AR. Mobile networks.

The usage of multi-path, multi-tier $360^{\circ}$ video streaming solutions are created to deal with both bandwidth and viewer motion.

5G virtualized architecture focused on a network func-
User and mobile node independence rendered changes in network availability undetectable to the user.

\section{Ascertaining huge opera-}

tional efficiency improvements over state-of-the-art caching and $360 \circ$ video representation techniques is extraordinarily promising.

The key role of edge computing in the effective deployment of AR apps.

Multimodal $360^{\circ}$ video streaming solutions for $5 \mathrm{G}$ wireless networks. Use a $5 \mathrm{G}$ network to its full capacity and deal with bandwidth volatility.

Better control of heterogeneity and service scalability are provided by service decoupling. tion which might relieve bandwidth constraints of immersive application scenarios.

\section{[123] AR and VR. Network services.}

\begin{tabular}{lll}
\hline $4-$ & Network & Scaling \\
\hline
\end{tabular}

[124] Orion

RAN slicing.

$\begin{array}{lll}\text { [125] Slice Alloca- } & \begin{array}{l}\text { Network resource } \\ \text { tion. }\end{array} & \text { Allocation. }\end{array}$

a RAN slicing technology that enables the dynamic onthe-fly virtualization of base stations, as well as the customisation of slices to match their particular service needs, was unveiled.

Solves an actual problem by combining several infrastructure providers.

SuperFlex, a network slicing architecture that deliv-

[126] SuperFlex. $\quad \begin{aligned} & \text { Network architec- } \\ & \text { tures. }\end{aligned}$ ers tailor-made treatment for subscribers without increasing capital and operating expenses.
Potential feature that might be used to relieve network resource utilisation.
Real-world traces of the 5G wireless network and user FoV analysis can be utilised to direct the design of future $360^{\circ}$ video streaming systems in 5G networks.

The datasets were actual, with tag information focused on the needs of users.
A low-powered yet flexible RAN virtualization tool for LTE was built as a proof of concept.
Orion is built for single-RAT setups but might be expanded to handle multi-RAT situations.
A slice allocation mechanism proposed based on matching game theory.

A pluggable multidomain, dual-layered slicing based efficient, scalable, and extensible 5G wireless network architecture providing universal connectivity in a sliced environment.
Dynamic pricing and power levels on a slice.

Concentrate on efficient chaining across multiple slices while enforcing rigorous latency constraints. 
Table 2. Cont.

\begin{tabular}{|c|c|c|c|}
\hline Ref. & Technique & Core Term & Core Idea \\
\hline [127] & $\begin{array}{l}\text { Multi-service } \\
5 G \text { Network. }\end{array}$ & $\begin{array}{l}\text { Network manage- } \\
\text { ment. }\end{array}$ & $\begin{array}{l}\text { Described implementation } \\
\text { experiences when deploying } \\
\text { a small-scale multi-service } \\
\text { network prototype, used to } \\
\text { demonstrate some selected } \\
\text { advanced features of } 5 \mathrm{G} \\
\text { Networking. }\end{array}$ \\
\hline [128] & NSRA. & $\begin{array}{l}\text { Heterogeneous } \\
\text { Network. }\end{array}$ & $\begin{array}{l}\text { A cache-enabled content de- } \\
\text { livery system for the } 5 \mathrm{G} \\
\text { heterogeneous network uses } \\
\text { base stations and the macro } \\
\text { cell as connected servers to } \\
\text { host caches. }\end{array}$ \\
\hline
\end{tabular}

Proposed an implicit mutual authentication and key exchange with group anonymity via proxy reencryption on elliptic curve.

Solution Drawbacks

Two heterogeneous services over two independent slices namely, video streaming and $\mathrm{AR}$, showcasing key features such as multi-slice orchestration RAN slicing and support for local breakout.

5G heterogeneous system model method minimise the absolute gap between the data rate necessary and the rate obtained for all users in the system.

IMAKE-GA protocol secures distributed, the secure association between network slice NSC pairs. It employs proxy re-encryption utilising bilinear pairing on an elliptic curve.

A solution that introduces a preemption mechanism to cut low-latency traffic while addressing the issue of flexible traffic that necessitates a higher throughput is provided.

The suggested solution's primary acting element is PASS an inter-slice scheduler that supplies services to each traffic type based on SLA demands.

High-level architecture to tackle the URLLC and network slicing problems via a sign criteria, system components, and basic interactions.

Future studies will incorporate densely-populated urban network simulations with higherorder MIMO.

Software is based on open source components, and most of it is also released as open-source on public repositories.

This study outlines certain crucial architecture design ponents, and interaction that enable RAN slicing for URLLC.
The next step is to integrate this method with grant-less upscheduler has outdated views of the UE uplink. set the path for future research initiatives in this direction. link transmission schemes, as the

These crucial outstanding issues MEC system, focusing on de-

[131] URRLC. Architecting RAN parameters, system com-

TPresented Mosaic5G, a community-led consortium for sharing platforms, featurAgile and flex-

[132] ible service Mobile networks. platforms. ing the software components FlexRAN, LL-MEC, and JOX on top of OpenAirInterface platform.

Proposed an approach that reduces noise by applying dynamic CPU pinning coupled with load balancing dependent on dynamic network slicing.

Mosaic5G, a community-led partnership for platform sharing. It offers the software components FlexRAN, LL-MEC, JOX, and Store, all of which are designed to help produce an open-source $5 \mathrm{G}$ research environment.
TDemonstrated an end-toend framework for noisy neighbour situations, regardless of NFV deployment model.
Must employ tools that imitate the actual world infrastructure to gauge the framework's effect.
Perform offloading in a multichannel context. So, in order to optimise total system throughput, a solution is devised that incorporates power regulation and interference.

A possible solution is point-

Aimed to reduce energy con-

Network

[135] resources

SLAs.

allocation. sumption in the network by simultaneously solving the user association and backhaul routing problems. to-point networks with enormous bandwidth available in the mmWave frequency spectrum.
Work on a case when both base stations and backhaul lines are disabled to obtain even more energy savings. 
Table 2. Cont.

\begin{tabular}{|c|c|c|c|c|c|}
\hline Ref. & Technique & Core Term & Core Idea & Solution & Drawbacks \\
\hline [136] & QoS. & $\begin{array}{lr}\text { Network } & \text { re- } \\
\text { sources } & \text { alloca- } \\
\text { tion. } & \end{array}$ & $\begin{array}{l}\text { To assign resources to the } \\
\text { uplink transmitters will re- } \\
\text { sult in increased spectral ef- } \\
\text { ficiency and maximum data } \\
\text { rate for all users. }\end{array}$ & $\begin{array}{l}\text { Modeled heterogeneous } \\
\text { multi-tier networks with the } \\
\text { concept of stable matching. }\end{array}$ & $\begin{array}{l}\text { The proposed technique can be } \\
\text { expanded to the forthcoming } 5 \mathrm{G} \\
\text { cellular networks. }\end{array}$ \\
\hline [137] & SOGMS. & $\begin{array}{l}\text { Network perfor- } \\
\text { mance analysis. }\end{array}$ & $\begin{array}{l}\text { Applications have an impact } \\
\text { on the exponential growth of } \\
\text { multimedia services in mo- } \\
\text { bile networks. }\end{array}$ & $\begin{array}{l}\text { SOGMS approach is offered } \\
\text { for multimedia services, fo- } \\
\text { cusing on efficient utilisation, } \\
\text { system capacity, and sustain- } \\
\text { ability requirements. }\end{array}$ & $\begin{array}{l}\text { Future } 5 \mathrm{G} \text { networks should look } \\
\text { to put in place proactive edging } \\
\text { caching with video popularity to } \\
\text { help with the users' QoE during } \\
\text { video delivery. }\end{array}$ \\
\hline
\end{tabular}

6- Smart Grid

[138] Future Smart Smart grid.
Grid.

Loss of ARPU in emerging countries in the context of $4 \mathrm{G}$ LTE and $5 \mathrm{G}$ networks are analysed.
More equipment and software applications will be required on the grid, including as sensors, faster processors, and stronger algorithms, in order to achieve greater efficiency and dependability in the distribution system.

Demonstrated how urban growth impacts $5 \mathrm{G}$ network coverage and quality of service.
Issues around standardisation, interoperability, security, and of course, cost are among the growing $5 \mathrm{G}^{\prime}$ 's priorities, but the longterm rewards are enticing.

Need to simulate seasonal effects like vegetation, rain, and snow. It incorporates data on travel patterns and simulations of city expansion.

An overview of the security and privacy issues associated with key 6G technologies is presented in Table 4.

Table 3. The possible services, core idea, and solutions uses of the existing Techniques.

\begin{tabular}{|c|c|c|c|}
\hline & Core Idea & Limitations & Ref. \\
\hline $1-$ & Network Resource Management & & \\
\hline Framework & $\begin{array}{l}\text { Dynamic network's mode selection method. Also Dis- } \\
\text { tributed core network architecture for future cellular net- } \\
\text { works. }\end{array}$ & $\begin{array}{l}\text { Framework deployment,control,management,monitoring, analyt- } \\
\text { ics, and security in } 5 \mathrm{G} \text { test beds. Also provides an emulator of } 5 \mathrm{G} \\
\text { infrastructures,which can accurately replicate } 5 \mathrm{G} \text { infrastructure. }\end{array}$ & $\begin{array}{l}{[79,82,92,95,96,99,103,106,} \\
107]\end{array}$ \\
\hline Performance & $\begin{array}{l}\text { Build a virtualized architecture for dynamic delivery of } \\
\text { services, QoS, and increased resource performance. Also, } \\
\text { Proposing a 5G platform-oriented solution amid alternative } \\
\text { authentication and authorisation techniques. }\end{array}$ & $\begin{array}{l}\text { All of these functionalities were implemented using modules } \\
\text { within the OpenStack cloud manager. }\end{array}$ & $\begin{array}{l}{[80,81,83,87,89,90,93,97,98,} \\
100,104]\end{array}$ \\
\hline $\begin{array}{l}\text { Network } \\
\text { compo- } \\
\text { nents }\end{array}$ & $\begin{array}{l}\text { Internet system using SDN in the core of the cellular net- } \\
\text { work and MEC in multilevels. Cognitive cellular network- } \\
\text { oriented wireless spectrum management technique, based } \\
\text { on cognitive radio technology. }\end{array}$ & $\begin{array}{l}\text { Delivered up to } 2 X \text { more successful delivery and incurs just a } \\
\text { tenth of the network overhead. Open-source software ecosys- } \\
\text { tem promotes concepts while sparking conversation on difficult } \\
\text { practical elements of multi-administrative. }\end{array}$ & $\begin{array}{l}{[84-86,88,91,94,101,102,} \\
105]\end{array}$ \\
\hline $2-$ & Security Approaches & & \\
\hline Framework & $\begin{array}{l}\text { To integrate security into the slice life cycle, effecting } \\
\text { the administration and orchestration of the virtualiza- } \\
\text { tion/softwarization architecture. }\end{array}$ & $\begin{array}{l}\text { Security architecture is made up of built-in security features based } \\
\text { on the ability to combine enforcement and monitoring operations } \\
\text { within the software-defined network infrastructure. }\end{array}$ & {$[110,112,114,115]$} \\
\hline Performance & $\begin{array}{l}\text { Precomputation-based digital signature creation techniques } \\
\text { and three-dimensional optimizations DPKI technique, pro- } \\
\text { tocol, and cryptographic scheme. }\end{array}$ & $\begin{array}{l}\text { Examine cryptography-backed authentication mechanisms to pre- } \\
\text { vent adversaries from enticing unsuspecting cellular devices to } \\
\text { connect to malicious base stations. }\end{array}$ & {$[111,118]$} \\
\hline $\begin{array}{l}\text { Network } \\
\text { compo- } \\
\text { nents }\end{array}$ & $\begin{array}{l}\text { Virtual to physical address encapsulation empowers mo- } \\
\text { bility capabilities, and mobility is implemented through a } \\
\text { flow table entry. }\end{array}$ & $\begin{array}{l}\text { User and mobile node independence rendered changes in network } \\
\text { availability undetectable to the user. }\end{array}$ & {$[108,109,113,116,117]$} \\
\hline $3-$ & Augmented Reality & & \\
\hline Framework & $\begin{array}{l}\text { 5G virtualized architecture focused on a network function } \\
\text { which might relieve bandwidth constraints of immersive } \\
\text { application scenarios. }\end{array}$ & $\begin{array}{l}\text { Potential feature that might be used to relieve network resource } \\
\text { utilisation. }\end{array}$ & {$[119,121,123]$} \\
\hline Performance & $\begin{array}{l}\text { The usage of multi-path, multi-tier } 360^{\circ} \text { video streaming } \\
\text { solutions are created to deal with both bandwidth and } \\
\text { viewer motion. }\end{array}$ & $\begin{array}{l}\text { Multimodal } 360^{\circ} \text { video streaming solutions for } 5 \mathrm{G} \text { wireless net- } \\
\text { works. Use a } 5 \mathrm{G} \text { network to its full capacity and deal with band- } \\
\text { width volatility. }\end{array}$ & [122] \\
\hline $\begin{array}{l}\text { Network } \\
\text { compo- } \\
\text { nents }\end{array}$ & $\begin{array}{l}\text { An effective representation method for constructing the } 360 \\
\text { films is needed in order to avoid sending a huge } 360 \text {. }\end{array}$ & $\begin{array}{l}\text { Ascertaining huge operational efficiency improvements over state- } \\
\text { of-the-art caching and } 360 \circ \text { video representation techniques is } \\
\text { extraordinarily promising. }\end{array}$ & [120] \\
\hline
\end{tabular}


Table 3. Cont.

\begin{tabular}{|c|c|c|c|}
\hline & Core Idea & Limitations & Ref. \\
\hline 4- & Network Scaling & & \\
\hline Framework & $\begin{array}{l}\text { 5G Solves an actual problem by combining several infrastructure } \\
\text { providers. }\end{array}$ & A slice allocation mechanism proposed based on matching game theory. & {$[125,126,128,132,133]$} \\
\hline Performance & $\begin{array}{l}\text { RAN slicing technology that enables the dynamic on-the-fly virtu- } \\
\text { alization of base stations, as well as the customisation of slices to } \\
\text { match their particular service needs, was unveiled. }\end{array}$ & $\begin{array}{l}\text { A low-powered yet flexible RAN virtualization tool for LTE was built as } \\
\text { a proof of concept. }\end{array}$ & {$[124,129]$} \\
\hline $\begin{array}{l}\text { Network } \\
\text { compo- } \\
\text { nents }\end{array}$ & $\begin{array}{l}\text { Described implementation experiences when deploying a small- } \\
\text { scale multi-service network prototype, used to demonstrate some } \\
\text { selected advanced features of } 5 \mathrm{G} \text { Networking. }\end{array}$ & $\begin{array}{l}\text { Two heterogeneous services over two independent slices, namely, video } \\
\text { streaming and AR, showcasing key features such as multi-slice orchestra- } \\
\text { tion RAN slicing and support for local breakout. }\end{array}$ & {$[127,130,131]$} \\
\hline 5- & Resource Allocation & & \\
\hline Framework & $\begin{array}{l}\text { Study the topic of underutilised 5G heterogeneous network spec- } \\
\text { trum resources. }\end{array}$ & $\begin{array}{l}\text { Perform offloading in a multi-channel context. So, in order to optimise } \\
\text { total system throughput, a solution is devised that incorporates power } \\
\text { regulation and interference. }\end{array}$ & [134] \\
\hline Performance & $\begin{array}{l}\text { To assign resources to the uplink transmitters will result in in- } \\
\text { creased spectral efficiency and maximum data rate for all users. } \\
\text { Modeled heterogeneous multi-tier networks with the concept of } \\
\text { stable matching. }\end{array}$ & $\begin{array}{l}\text { The proposed technique can be expanded to the forthcoming } 5 \mathrm{G} \text { cellular } \\
\text { networks. }\end{array}$ & {$[136,137]$} \\
\hline $\begin{array}{l}\text { Network } \\
\text { compo- } \\
\text { nents }\end{array}$ & $\begin{array}{l}\text { Applications have an impact on the exponential growth of multi- } \\
\text { media services in mobile networks. }\end{array}$ & $\begin{array}{l}\text { SOGMS approach is offered for multimedia services, focusing on efficient } \\
\text { utilisation, system capacity, and sustainability requirements. }\end{array}$ & [135] \\
\hline 6- & Smart Grid & & \\
\hline Framework & $\begin{array}{l}\text { Loss of ARPU in emerging countries in the context of } 4 \text { G LTE and } \\
5 \mathrm{G} \text { networks are analysed. }\end{array}$ & $\begin{array}{l}\text { More equipment and software applications will be required on the grid, } \\
\text { including as sensors, faster processors, and stronger algorithms, in order } \\
\text { to achieve greater efficiency and dependability in the distribution system. }\end{array}$ & [138] \\
\hline Performance & Designed a tool to test the spatial resiliency of $5 \mathrm{G}$ networks. & $\begin{array}{l}\text { Demonstrated how urban growth impacts } 5 \mathrm{G} \text { network coverage and } \\
\text { quality of service. }\end{array}$ & [139] \\
\hline
\end{tabular}

Table 4. Overview of the main security and privacy issues in key 6G technologies.

\begin{tabular}{|c|c|c|}
\hline $\begin{array}{l}\text { Security and Privacy } \\
\text { Issues }\end{array}$ & Key Technology Contribution & Ref. \\
\hline $1-$ & $\mathrm{THz}$ & \\
\hline Authentication & attacks that can exist in the 5G.then the security requirements in the $5 \mathrm{G}$ & $\begin{array}{l}{[5,46,48,116,201,207,} \\
208]\end{array}$ \\
\hline Privacy & $\begin{array}{l}\text { Describes these threats and sketches future research on how to mitigate } \\
\text { the security concerns that a modern distributed control infrastructure } \\
\text { poses. }\end{array}$ & {$[7,53,109,111,115]$} \\
\hline Malicious behavior & Privacy and confidentiality issues. & {$[29,68,75,114]$} \\
\hline $2-$ & AI & \\
\hline Communication & $\begin{array}{l}\text { vision of AI-enabled } 6 \mathrm{G} \text { system, the driving forces of introducing } \mathrm{AI} \text { into } \\
6 \mathrm{G} \text { and the state of the art in machine learning. }\end{array}$ & $\begin{array}{l}{[8,10,22,62,112,113,} \\
203,204]\end{array}$ \\
\hline Privacy & $\begin{array}{l}\text { Secure ML structure, or the correct application of ML, can protect privacy } \\
\text { in } 6 \mathrm{G} \text {. }\end{array}$ & {$[9,11,21,37,61]$} \\
\hline Access control & $\begin{array}{l}\text { Identify fascinating services and use-cases of } 6 \mathrm{G} \text {, which can not be sup- } \\
\text { ported by } 5 \mathrm{G} \text { appropriately.. }\end{array}$ & {$[49,60]$} \\
\hline 3- & Quantum communication & \\
\hline Communication & $\begin{array}{l}\text { Highlight the use cases and applications of the proposed 6G networks in } \\
\text { various dimensions. }\end{array}$ & {$[13,65,118,209]$} \\
\hline 4- & Blockchain & \\
\hline Communication & Challenges and canvassed the key role of blockchain. & {$[14,44,47,66]$} \\
\hline 5- & Molecular communication & \\
\hline Privacy & $\begin{array}{l}\text { Preserving the privacy of the users is the primary concern of mobile and } \\
\text { service providers. }\end{array}$ & $\begin{array}{l}{[39,41,42,52,54,56,57,} \\
67,202]\end{array}$ \\
\hline Encryption & $\begin{array}{l}\text { Several challenges pertaining to resource allocation, task offloading, en- } \\
\text { ergy efficiency, latency reduction, fairness and security based } 6 \mathrm{G} \text { enabled } \\
\text { massive IoT. }\end{array}$ & {$[69,70]$} \\
\hline
\end{tabular}


Table 4. Cont.

\begin{tabular}{llll}
\hline $\begin{array}{l}\text { Security and } \\
\text { Issues }\end{array}$ & Privacy & Key Technology Contribution & Ref. \\
\hline Authentication & Securing 5G hetNets using mutual physical layer authentication & {$[117,200]$} \\
\hline $6-$ & VLC & {$[43,45,63,64,198]$} \\
\hline Communication & Overview of the first five generations of wireless systems. & $\begin{array}{l}\text { how to ensure seamless operability (including, but not limited to, autho- } \\
\text { rization, security, service provisioning, accounting, etc.). }\end{array}$ & {$[76,108,206]$} \\
\hline Malicious behavior & lat & \\
\hline
\end{tabular}

\section{Internet of Things (IoT)}

The IoT is a network concept that allows different objects to connect with one another. These gadgets are integrated with embedded technology, which allows them to communicate with both internal systems and the outer environment at the same time. We've done an explanatory evaluation of the use of the internet of things as the foundation for a transition strategy toward the development of 5G and 6G communications networks and technologies. The ninety-five articles in this category were separated into five subcategories, each of which had a single article.

\subsection{Resource Allocation}

IoT networks may be setup with a control centre to give centralised control to be compatible with existing cellular mobile networks such as the 3GPP LTE/LTE-A. IoT networks, on the other hand, can be structured in a dispersed form for greater flexibility and scalability. As a result, the frameworks of both centralised and distributed IoT networks will be addressed in this section. Some publications [140-144] proposed a service slicing technique that may divide network resources between distinct service slices with greater flexibility. It provides an ultra-reliable low-latency service for URLLC applications, as well as low to medium latency services for $\mathrm{xMBB}$ and mIoT applications in future $5 \mathrm{G}$ networks.

Implement a set of parallelism-aware compile tools for swift to take use of parallelism while developing apps. The performance of the multi-protocol strategy and the characterization of bottlenecks in the evaluated protocols are among the contributions. Other papers $[145,146]$ offered a cross-layer MAC and physical layer solution for low-power, low-bitrate devices with a long communication range and short access delay. By modifying the edge network, primarily eNodeB, the proposed protocol will combine IoT traffic with traditional systems. Other articles $[147,148]$ discussed the existing proposed spectrum for 5G and the internet of things, as well as licenced, unlicensed, and licenced shared access schemes for future generations, as well as deployment scenarios for countries and effective ranges to achieve better results for harmonised spectrum. Also, investigate and illustrate how to compensate for the uncertain and time-varying latency introduced by a $5 \mathrm{G}$ mobile network when controlling a latency-sensitive plant.

For the baseband units pool in CRAN, three studies [149-151] developed efficient cache management strategies. It uses an exponential decay approach to hold recently frequently requested records in cache and AHP to allow different degrees of mobility and QoS. To make the marriage between mobile business processes and NFV MANO more fruitful, we'll use our understanding of how QoS limitations change in specific sets of operations.

Several papers [152-154] proposed a distributed latency-aware data processing model in which fog computing enabled GWs dynamically exchange processing and storage capability information and probabilistically forward data to neighbouring GWs or the Cloud only when local processing or storage capacity is limited. The suggested technique reduces overall response time and lowers the total cost significantly. Cache management strategies are designed and evaluated in few publications [155-158]. A probability-based scoring method, a hierarchical or tiered, approach, and improvements to previously existing 
approaches are among the algorithms. The control plane functions are self-contained in the sense that they can be run independently. By handling this signalling locally, the control functions can use the distributed cloud to manage the massive amount of control signalling.

Many articles [159-162] advocated integrating a message broker and Publish/Subscribe messaging transport using lightweight Internet protocols. To reduce the need for microcontroller in IoT devices, the design was validated using firmware. Demonstrate that the proposed technique improves the system's energy efficiency as compared to an IP-based implementation. The suggested technique also provides a framework for implementing application-level access control and QoS support. In the context of the 5G environment, three studies [163-165] suggested revolutionary centralised energy balancing uneven concentric chain clustering technique for the IoT system. To lessen the stress on the cluster head, it incorporates a probability-based suboptimal multi-hop path selection algorithm that adjusts the cluster head diameter based on the energy level and distance to the base station.

Three publications [166-168] focused on the resource allocation challenge in FiWi access networks supporting IoT services presented a one-of-a-kind system running at $4.8 \mathrm{GHz}$ frequency for measuring the WCI of post-surgical falls and other human activities. The total system makes use of low-cost wireless hardware such as a network interface card, an RF signal generator, an omnidirectional antenna, and a desktop PC. ML algorithms such as SVM, NB, and DT are used to classify falls and other human behaviours. The accuracy rises at a rate of $4 \%$ to $5 \%$ per year.

Two papers $[169,170]$ presented a collaborative distributed Q-learning approach for resource-constrained MTC devices to enable them to locate unique RA slots for their transmissions, hence reducing the number of possible collisions.

Two papers [171,172] proposed a resource allocation for Industry 4.0 based on softwaredefined networking and network function virtualization technologies, machine learning tools, and the slicing paradigm, in which each slice of the network is dedicated to a category of services with similar QoS requirements.

Another paper [173] described a unique MTC architecture that provides NFC as a service rather than collecting raw large-volume MTC data. Different modules of the communication infrastructure are orchestrated into a directed network topology for a given application demand, and each module is assigned an appropriately defined atomic function over the input data, allowing the desired global network function to be evaluated over MTC data and a requested MTC-NFC service to be delivered. One paper [174] proposed a network architecture that combines green IoT and 5G networks. To analyse massive data in 5G networks, we presented a human-enabled green IoT system. Green IoT is achieved by grouping together various mobile devices.

Two articles $[175,176]$ in 6G-based edge computing, SSPS was proposed for MCS. In addition to task acceptance rate and weighted schedulability, QoS is taken into account in SSPS to improve the system's service quality. Jobs are assigned to each processor via the SSPS, and some tasks can be moved to other processors as quickly as possible.

Three papers [177-179] proposed a low-cost sensor array design technique based on irregular subarrays, which divides the practical application challenge into two subproblems based on scene scale. To address these issues, other articles [180] offer practical recommendations such as deep Q-learning and federated learning-based transceivers, blockchainbased secure business models, homomorphic encryption, and distributed-ledger-based authentication systems.

Three publications [181-183] provide a process-oriented optimization methodology for simultaneously assigning sub-channels, transmit power, and hovering durations that takes into account the entire flight process of UAVs and only needs slowly-varying large-scale channel state information (CSI).

Another article [184] describes a two-tier matching method that combines the GaleShapley-based matching algorithm for users and HAPs with the random path to a pairwisestable matching technique for HAPs and satellites. The proposed algorithms' effectiveness is demonstrated by numerical results. 
A solution based on an integrated DL algorithm was offered in several articles [185-188]. The positive samples are extended to balance the number of positive and negative samples, and CT images are pre-processed using image clipping, normalisation, and segmentation.

An ACO strategy is described in two studies [189,190], prompted by the requirement to secure 6G IoT networks by adopting various objectives and employing transaction deletion to secure secret and sensitive information.

Many articles [191-194] in big data-driven and nonparametric model supported by $6 \mathrm{G}$ is suggested to extract comparable traffic patterns over time for accurate and efficient short-term traffic flow prediction in enormous IoT, which is mostly based on time-aware LSH, which is mainly based on time-aware LSH.

To characterise the spatiotemporal relationships among heterogeneous data, the authors of [195] proposed approach employs a multidimensional data relationship diagram. Then, to reduce the impacts of noise on sensor data and aid in the detection of anomalies, an autoregressive exogenous model is used. Finally, the method generates a CCoV, which can be used to detect high-value sensing devices and enable huge IoT with 6G using the data's unique patterns.

The logistics mode is optimised and improved, and the agricultural e-commerce common delivery method is presented in two publications $[196,197]$ based on an examination of the distribution efficiency of the current mainstream agricultural goods logistics distribution mode. RoF is one of the most promising enablers for $6 \mathrm{G}$ IoT systems due to its excellent flexibility and efficiency.

\subsection{Security}

This section details the research that looked into the security of IoT design properties, architecture, and protocols, as well as their practicality in terms of security.

Four authentication techniques were evaluated in two papers [198,199], and the discussion of the results led to the conclusion that each protocol had advantages and disadvantages and that these analyses should be followed. The security elements of this research team's new 5G-IoT architecture, which was just designed. At each tier of the 5GIoT architecture, classify potential security attacks in the context of smart city applications, a security taxonomy for 5G-IoT architecture. This taxonomy is made up of five layers that are used to defend against the attacks that have been examined as well as to secure the privacy of customers.

The importance of device capabilities information supplied for 5G devices in creating security associations between the device and the network was investigated in two studies $[200,201]$. Introduce three new types of attacks against cellular devices that take advantage of unsecured device capabilities information in 4G and forthcoming 5G networks: identity attacks, bidding down attacks, and battery drain attacks.

Three articles [202-204] provide a solution that relieves the IoT provider's burden of device identity management while also lowering operational costs. Open-source software for LTE, identity management, and IoT is used to implement the solution. Integrate adaptive wormholes with CRKE, a lightweight security solution for IoT devices that may be even more crucial for the 5G tactile Internet and its embedded low-end devices.

A framework for a road-side infrastructure-independent traffic event detection system based on 5G communications, big data analytics, and augmented reality was proposed in another article [205]. The preliminary results of a traffic detection algorithm have been provided, as well as a direction for future research. An efficient physical layer identifies spoofing attack detection mechanism for IoT was proposed in another article [206]. A twostep detection approach exploits the sparsity of the virtual channel in Mm-Wave and Massive MIMO 5G communication. The technique finds anomalies in the first stage by angles of arrival (AoA) and path gains of all IoT devices in the VCS at the same time.

Another article [207] the EKF approach, is used initially to forecast future harvesting power. Then, in each energy-aware cycle, create a mathematical model to compute the 
necessary energy of various security measures and select the maximum level of protection that can match service requirements while avoiding energy exhaustion.

The goal of this effort, according to one publication [208], is to build a safe and secure CDS in a wireless network that runs over an SB, which will provide users with a safer and more efficient environment for browsing the Internet, sharing, and handling enormous amounts of data in the fog. There were two sorts of servers in this CDS: one cloud server and one edge server.

One article [209] explored the security and privacy issues that the available 6G specifications and possible 6G applications may provide. Give the reader an overview of the standardisation activities and research programmes related to 6G security. Security concerns with 6G enabling technologies such as DLT, physical layer security, distributed $\mathrm{AI} / \mathrm{ML}, \mathrm{VLC}, \mathrm{THz}$, and quantum computing were examined in particular.

\subsection{Smart Cities}

This section details the research that was done to examine smart home IoT design features, architecture, and feasibility in terms of 5G and 6G.

Two publications [210,211] proposed upgrading current infrastructure to host cloudlets on a city-wide scale, allowing smart cities to provide new services to inhabitants. The findings suggest that updating a small number of access points can result in city-wide cloudlet coverage.

Three articles [212-214] look at the latest cooperative MIMO antenna technologies, which are the bridging technologies to $5 \mathrm{G}$, by first looking at the benefits of cooperative MIMO in smart city applications like smart transportation, home automation, and security, and then evaluating the impact of cooperative MIMO technologies on achieving higher spectral efficiency.

Some articles [215-220] point to unsolved issues and emphasise the necessity for piloting with 5G apps in order to better understand the setup of 5G networks and the use of applications in a variety of vertical industries. In a typical smart house, you can see numerous topologies and configurations employing ZigBee communication. Dedicated to smart homes and, in particular, ZigBee as a smart data collection element. In addition, the IoT SoftRadio tool is introduced, and NB-IoT UEs sharing scenarios are summarised.

Finally, using the current NB-IoT radio performance management framework. Discuss the state of the art in the topic of call admission control in 5G networks. Which study will be carried out in order to propose and create an algorithm for admission control modelling in the situation of New Radio access, namely NR 5G? An RNN-based arrival angle predictor was proposed in two studies [221,222] to predict the particular communication location of UAVs in $5 \mathrm{G}$ IoT networks. To make the training process easier and more effective, a grid-based coordinate system is used during data preparation.

One article [223] aimed to chart the major directions and scope emerging dimensions inherent to 6G technology, including digital twins and immersive realities that, development and application of $6 \mathrm{G}$, noting its numerous potential benefits in tackling a variety of urban challenges, including environmental dimensions, needs to be accompanied by political policy agendas supporting sustainability transnationally.

The authors of [224] designed a system based on 6G network communication standards for the next IoT level. Water flow management, wind shield control, security concerns, and carbon dioxide limitation via adaptive ventilation are all effective with the proposed control approach. The infrastructure has been developed to support the new 6G communication standard, which will improve efficiency and data flow at the end-user device and local area level.

A framework for DAIaaS provisioning for IoE and 6G contexts was proposed in one study [225]. Because the actual training and inference computations are divided into smaller, concurrent computations tailored to the degree and capacity of resources accessible with cloud, fog, and edge layers, the AI service is called "distributed". To study the design choices and performance bottlenecks of DAIaaS, multiple DAIaaS provisioning settings for distributed training and inference are provided. 
Another article [226] offered a conceptual design for job off-loading and resource allocation dubbed the typical model. Second, expand on the traditional paradigm to include intelligence for work off-loading and resource allocation, produce particular research questions in order to build and evaluate the performance of various units within the above-mentioned models in order to accommodate technological improvements such as the employment of $\mathrm{AI}$ in the $6 \mathrm{G}$ wireless communication era.

\subsection{Virtual Reality}

One of the most critical high-throughput application-level requirements of $6 \mathrm{G}$ is AR/VR. When AR/VR can be utilised more efficiently, conveniently, and without regard to location, it will encourage the rapid creation of AR/VR services and applications, which will, in turn, encourage the quick development and maturity of AR/VR devices.

One publication [227] described a case study in an oilfield that looked at the MAS paradigm and the possibilities for new development with 5G. Production management and related tasks, such as production monitoring, cost analysis, maintenance scheduling, inventory control, and supply chain management, are the emphasis of the proposed MAS model. The ultimate VR 360 that satisfies human eye fidelity is the subject of another article [228]. The ultimate VR 360 requires a 1.5 Gbps downlink and a 6.6 Gbps uplink for live streaming, with a round-trip time of less than 8.3 milliseconds. Examine whether the most cutting-edge wireless communication technologies are capable of supporting the ultimate VR 360 experience.

In one publication [229], the authors listed key enabling technologies for an intelligent and open 6G network, all of which included 3C convergence. 3C-based spectrum management, radio channel design, delay-aware transmission, wireless distributed computing, and network self-evolution are among the subjects covered by these technologies.

Other unique VLC concepts, such as interactive VLC, light-based IoT, living surfaces, and optical communications through bio-tissues, are given and discussed in another paper [230]. These extremely interesting technologies can be applied to the selected 6G verticals, hence expanding $6 \mathrm{G}$ research into new sectors.

\subsection{Other Machine Learning}

ML-based intelligent application scenarios will enable rich, heterogeneous connections, as well as large data storage and processing.

A concept for such a traffic control system was proposed in three publications [231-233]. Its components are described as well, including 5G networking, RFID-based parking space monitoring, and cloud services for supervisory control and ML. For the social sustainability of smart cities, joint verification of smart user profiles is necessary. The collaborative architecture delegated the detection of potential identity theft to other smart users who are the potential victim's social platform contacts.

Another paper [234] presented an uncoordinated access protocol for huge connectivity that takes advantage of a large number of antennas at the base station and is likely to be widely used in cellular networks. A large number of IoT devices can transmit data without any prior scheduling procedure using the proposed approach, which consists of a sparse frame structure and receiver processing based on sparse dictionary learning.

\section{Merging Energy and IoT in 5G, 6G}

There have been several recent attempts in implementing strategies to handle various difficulties in wireless communications in the direction of merging energy and IoT in 5G, 6G, and beyond networks. This category's 104 articles were broken into two subcategories.

(1) Table 5 Analysis of ML Usage in Incorporating Energy and IoT in 5G and 6G.

(2) Table 6 Summary of Machine Learning-Based schemes for 5G and 6G mobile and wireless communications technology [235-238].

The primary objective of this article is to discuss the advantages and disadvantages of various ways of learning in the context of future $5 \mathrm{G}$ and $6 \mathrm{G}$ communications. The article 
discusses detection and learning approaches in the context of energy-efficient 5G and 6G networks. It was demonstrated that the volume of examined context-awareness information is huge. Table 5 summarises the possible services, core idea, and limitations uses of machine learning in 5G and 6G-based energy technologies.

Table 5. Analysis of ML Usage in Incorporating Energy and IoT in 5G and 6G.

\begin{tabular}{|c|c|}
\hline ML & 5G,6G-Based Applications Energy and IoT \\
\hline ANN & $\begin{array}{l}\text { Their primary advantages have been identified, and the feasibility of their implementation in tackling the problem of accurate attack } \\
\text { detection in } \mathrm{m} 2 \mathrm{~m} \text { adhoc self-organizing networks has been assessed [239]. }\end{array}$ \\
\hline DL & looked into the challenges involved in trying to blend MEC with C-RAN, specifically regarding cloud resources. energy-reduction [240,241]. \\
\hline DL & $\begin{array}{l}\text { Implemented a modern vehicle-internet infrastructure-aware ML architecture. It has made managing network slicing difficult in the context } \\
\text { of 5G-enabled networks [242]. }\end{array}$ \\
\hline DL & $\begin{array}{l}\text { Has provided an APP-SON system which optimises } 4 \mathrm{G} / 5 \mathrm{G} \text { network performance and user QoE. App-son offers a scalable big data platform } \\
\text { for targeted optimization by evaluating cell application features in temporal space }[243,244] \text {. }\end{array}$ \\
\hline DL & proposed to apply DL algorithm based on 5G-V2X for AI-based 5G base station allocation for platooning cars [245,246]. \\
\hline RL & $\begin{array}{l}\text { Found an E2E network slicing system that uses deep reinforcement learning to manage many resources to efficiently generate network } \\
\text { slices [247-249]. }\end{array}$ \\
\hline Bayesian & $\begin{array}{l}\text { Bayesian learning was used to identify the broadcast signals for LA-CDMA uplink access. Using the sparse signals, this proposed strategy } \\
\text { leverages our ignorance about user activities. Furthermore, introduce Gaussian mixture model approach to calculate the transmitted signals } \\
\text { [250]. }\end{array}$ \\
\hline ML & $\begin{array}{l}\text { Proposed an online learning detection approach for the NOMA uplink. Build an online adaptive filter in the sum space of linear and } \\
\text { Gaussian reproducing kernel Hilbert spaces. So, this approach is robust to dynamic wireless networks that can degrade the efficacy of a } \\
\text { nonlinear adaptive filter [251]. }\end{array}$ \\
\hline ML & $\begin{array}{l}\text { Proposed the first online learning method designed to aid beam selection in mmWave vehicular systems. in particular, see this as a } \\
\text { multi-armed bandit problem. Next, developed a lightweight context-aware online learning algorithm, known as FML, with proven } \\
\text { performance and assured convergence }[252,253] \text {. }\end{array}$ \\
\hline ML & $\begin{array}{l}\text { Investigated healthcare scenarios where communication is conducted at THz frequencies. Combined with an ML mechanism, THz } \\
\text { communications protocols result in fewer signal route losses in the system [254-256]. }\end{array}$ \\
\hline ML & $\begin{array}{l}\text { The new algorithm that incorporated a change in the hidden layer of the RNN is known as GRUs. The RNN-GRU model is used for } \\
\text { determining whether or not to enable the VC mode. Received signal strength measurements were utilised to train the RNN-GRU model } \\
{[257,258] \text {. }}\end{array}$ \\
\hline ML & $\begin{array}{l}\text { Most notable similarities between the cloud-fog node architecture and the human brain-spinal cord-nerve network model involve the } \\
\text { presence of fog nodes [259-261]. }\end{array}$ \\
\hline RL & $\begin{array}{l}\text { The user association problem is tackled with a reinforcement learning method that considers content placement profiles and frontal } \\
\text { constraints [262-264]. }\end{array}$ \\
\hline ML & $\begin{array}{l}\text { Detected the mood of patients by implementing an intelligent real-sense camera system prototype. ML, an SVM, and the RealSense facial } \\
\text { detection system can be utilised to track patient demeanour for pain monitoring [265]. }\end{array}$ \\
\hline ML & $\begin{array}{l}\text { Developed a lightweight context-aware online learning method called FML with performance and convergence guaranteed. Using coarse } \\
\text { location information and aggregating the data, FML learns from and adapts to its environment. Furthermore, propose a standard-compliant } \\
\text { protocol that utilises the existing cellular network architecture and the future } 5 \mathrm{G} \text { characteristics [266-268]. }\end{array}$ \\
\hline ML & $\begin{array}{l}\text { Focused on designing and detecting RA preamble for 6G IoT satellite-assisted 6G an energy-conscious course for big IoT devices [269]. } \\
\text { The drone follows the shortest path across a connected graph. This path decides the visiting order of devices. According to system } \\
\text { identification theory, and with the use of neural networks, the model is constructed based on the results [270,271]. }\end{array}$ \\
\hline NN & FA neural network combined with system identification theory is used, and the model is created based on the gathered data [272]. \\
\hline ML & Enriched standard HetNets with a user-centric ML dimension [273]. \\
\hline
\end{tabular}

Table 6. Summary of Machine Learning-Based Schemes for 5G and 6G Mobile.

\begin{tabular}{llll}
\hline ML & Core Idea & Limitations & Ref. \\
\hline 1- & Networks Architectures & $\begin{array}{l}\text { How to increase the algorithm's accuracy, and hence its estimating } \\
\text { performance. }\end{array}$ & [274] \\
\hline ANN & Ensure the accuracy and simplify channel estimate. & N3AC scales to huge scenarios and is useful in real applications. & [275,276] \\
\hline ANN & Use SMDP for the decision-making. & $\begin{array}{l}\text { Converts probability distributions into images and applies as- } \\
\text { sumptions of conditional independence to decrease the computa- } \\
\text { tional load of probabilistic reasoning. }\end{array}$ & [277,278] \\
\hline BL & $\begin{array}{l}\text { Used for the forecast of throughput. Parameter estimation fore } \\
\text { casts future test results. }\end{array}$ & Only the simulation shows that NN. & [279] \\
\hline DL & Proposed a DNN-based AS/STSK MIMO system. & &
\end{tabular}


Table 6. Cont.

\begin{tabular}{|c|c|}
\hline ML & Core Idea \\
\hline DL & 5G-oriented architecture in which to analyse network traffic. \\
\hline DL & Using ML methods to minimise link failure at handover. \\
\hline DL & Reduce network load and availability. \\
\hline DL & $\begin{array}{l}\text { Create realistic synthetic data by exploiting the GAN's capacity to gener- } \\
\text { ate and separate data. }\end{array}$ \\
\hline DL & $\begin{array}{l}\text { Semantic edge cache optimization for multimedia services in } 5 \mathrm{G} \text {, which } \\
\text { online improves caching settings depending on user playing behaviour. }\end{array}$ \\
\hline ML & Verification of quality of experience. \\
\hline ML & $\begin{array}{l}\text { Distributing resources to high mobility users while utilising only their } \\
\text { position estimates. }\end{array}$ \\
\hline
\end{tabular}

Limitations

Ref.

Only TensorFlow was tested, and an in-depth comparison of deep learning frameworks would be essential.

It might enable cellular systems to escape threshold-based handover decisions and follow ML-based methods.

Need to include mimicking the created model in a real production environment once the $5 \mathrm{G}$ ecosystem is accessible for customers.

Proactive SON algorithms run by GAN generated synthetic CDRs.

Sharing the cache resource across many BSs is essential for heterogeneous networks.

Add more diverse and difficult scenarios, like deployment, client movement, and dynamic video and client attributes.

Training the ML unit of the proposed approach will help alleviate inaccurately position information availability-related performance loss.

Extend this inquiry to examine representative ML strategies for autonomic allocation and adjustment of computing and network resources on the basis of service requirements.

ML construction, deployment, operation, control, and management of slices.

ML 5G challenges and the importance of a changed management paradigm Next steps include actions such as checking the proposed algorithms at scale on commercial networks to validate the idea of adaptive SDN route planning in context.

ML MIMO mmWave-assisted downlink for multiple users and Massive

Compared ML-based with CVX-based optimal RRM.

ML It is possible to learn basic state representations, minimising the complexity of the fundamental network design requirements.

Comparative simulation results demonstrated the suggested decision framework in multi-agent settings.

ML Used ML to construct a framework for 5G network orchestration. ML approaches use Decision Maker building blocks using neural networks for optimization.

ML Allocation of the slice is explored and solved using ML approaches. Solve for scalability

ML Found features that alter NFP resiliency. Many of these features are

ML design and engineering features, therefore are difficult to measure.

ML Presented a comprehensive self-healing mechanism as an enabler for a ML truly holistic and resilient solution

ML Flow of thinking led to this assumption, which is then used to define the journey towards CAN and a functional design of a typical CAN system.

ML Research approaches for SON functions for anomaly detection, load balancing, and capacity optimization.

ML Plotted the locations of the drone-BSs and calculated the frequency reuse

ML factor based on an octahedron-shaped structure.

ML and blockchain can improve the resilience of aerial networks.

[307]

TL strategies will be researched in regards to self-healing, which focuses on applying corrective measures. tecture.

Next-generation SON networks should be built using ML-based usercentric techniques in order to achieve a linked eco-system with enhanced user experience.

Proposed approach drastically lowers the latency of drone-user UEs.

RL Feature-learning was applied to mine and understand the complex correlation between multi-RMIs and link quality.

RL Methodology is shown using a network simulation in ns-3 and the ML implementation in Python.

To anticipate the movement, apply strategy learning, and regard the location as the input. a destination, there is the potential to implement OCSP. Provided insight into the learning challenge of cognitive radio networks

RL and highlights methodologies such as RL used in cognitive radio networks.

$5 \mathrm{G}$ network slicing-based design that integrates with the ML-based CRN to maximise the use of the restricted spectrum.

Q-learning offers a perspective on D2D, and in combination with RLLCDC, offers a practical algorithm for decision making under uncertain network conditions.

RL Research towards a ML-based remote local (D2D) communicat
work focused on effective connectivity and minimum latency.

RF Designing a learning-based resource allocation strategy which incorporates simply position information is utilised. to inaccurate user positioning.

RF Cartel, a collaborative learning platform in edge clouds. Add other ML models to the system.

TL Presented an ML approach that enables semi-blind UL and DL decoupling in mixed sub-6 GHz/mmWave cell-free $5 \mathrm{G}$ networks.

$95 \%$ of accuracy in just a few training samples, with a quick and reliable solution to UL and DL decoupling in 5G networks. Evaluate the predictive performance of four significant predictors that are

SVM crucial to achieve mobile traffic management, manage mobility, and conserve energy in future cellular networks.

Study robustness of these predictors versus varying training dataset sizes. Benchmark performances base on mobility traces from network CDRs. and improving the proposed dependability assessment method.

Following positive DL assisted communication results, committed to

DL exploring a unique DL-based strategy for SCMA systems, motivated to increase BER performance.

Proposed schemes must be implemented and verified in a real environment system. An in-depth study of the structure of the learnt codebook may result in a higher decoding accuracy. 
Table 6. Cont.

\begin{tabular}{|c|c|c|c|}
\hline ML & Core Idea & Limitations & Ref. \\
\hline DL & $\begin{array}{l}\text { Important problems with regard to dynamic resource scheduling } \\
\text { design and offered a data-driven scheduling strategy. }\end{array}$ & $\begin{array}{l}\text { Additionally, improving the physical network's resource usage } \\
\text { while guaranteeing QoS. }\end{array}$ & {$[347,348]$} \\
\hline DL & $\begin{array}{l}\text { ANN models include feed-forward NN and radial basis function } \\
\text { neural networks. }\end{array}$ & $\begin{array}{l}\text { Validation of simulation findings has proven that ML algorithms } \\
\text { can be powerful analytical tools for future measurement-based } \\
\text { channel modelling. }\end{array}$ & {$[349,350]$} \\
\hline DL & $\begin{array}{l}\text { Path loss prediction with better generalisation using satellite pic- } \\
\text { tures can be done with twisted NN. }\end{array}$ & $\begin{array}{l}\text { Proposal stands to benefit from increased data since more infor- } \\
\text { mation can be used to quantify the generalisation reached. }\end{array}$ & {$[351,352]$} \\
\hline $\begin{array}{l}\text { K- } \\
\text { means }\end{array}$ & $\begin{array}{l}\text { Proposed two NN-based MLP models to aid in proactive auto- } \\
\text { scaling of VNFs using commercial traffic traces. }\end{array}$ & $\begin{array}{l}\text { To tackle the above mapping problem, ILP is able to solve it in } \\
\text { seconds rather than hours. }\end{array}$ & [353] \\
\hline NN & $\begin{array}{l}\text { Binomial distribution-based power coefficient allocation proce- } \\
\text { dure; Pascal's triangle. }\end{array}$ & $\begin{array}{l}\text { Adaptive modulation and coding techniques significantly en- } \\
\text { hanced the system's BER performance. }\end{array}$ & [354] \\
\hline NN & $\begin{array}{l}\text { Facilitate automatic network adjustment and intelligent resource } \\
\text { allocation optimization. }\end{array}$ & $\begin{array}{l}\text { Downlink communications and the interference-elimination re- } \\
\text { source allocation mechanisms that go along with it will be exam- } \\
\text { ined. }\end{array}$ & [355] \\
\hline 2- & Quality of Service (QoS) & & \\
\hline ML & $\begin{array}{l}\text { Results on how the model functions in the real world are pro- } \\
\text { vided. }\end{array}$ & N3AC scales to huge scenarios and is useful in real applications. & [317] \\
\hline ML & $\begin{array}{l}\text { To test the suggested framework architecture, a case study of QoS } \\
\text { anomaly root cause tracking was given. }\end{array}$ & $\begin{array}{l}\text { ML mechanisms, with the skills to train a computer to learn knowl- } \\
\text { edge and concepts from data, can react intelligently to changing } \\
\text { environments. }\end{array}$ & {$[318,319]$} \\
\hline ML & $\begin{array}{l}\text { Shows that ML can be integrated into an SDN controller to esti- } \\
\text { mate resource need and behave appropriately, which this one is } \\
\text { learning to implement by way of KPIs. }\end{array}$ & $\begin{array}{l}\text { Design and implement new QoE measures specifically related to } \\
\text { delay stall time, and the number of quality transitions. }\end{array}$ & {$[320,321]$} \\
\hline ML & $\begin{array}{l}\text { System, based on RL algorithms, allowing online learning of a dy- } \\
\text { namic and partially visible environment with delayed feedbacks. }\end{array}$ & $\begin{array}{l}\text { Improving the GOL architecture includes employing } \\
\text { bers and fuzzy RL. }\end{array}$ & [322-324] \\
\hline RL & $\begin{array}{l}\text { Developed a distributed approach for managing QoS radio re- } \\
\text { sources in a dense heterogeneous network. }\end{array}$ & To dynamically react to changing traffic types. & {$[330,331]$} \\
\hline 3- & Big Data & & \\
\hline ANN & $\begin{array}{l}\text { Tests show that CNN and RNN can find spatial and temporal } \\
\text { traffic information. }\end{array}$ & $\begin{array}{l}\text { More accurately projected traffic loads for proactive management } \\
\text { are found in } 5 \mathrm{G} \text { networks. }\end{array}$ & [356] \\
\hline DL & $\begin{array}{l}\text { Implies the unnecessary waste of valuable resources, which ulti- } \\
\text { mately disrupts operations. }\end{array}$ & $\begin{array}{l}\text { Implemented algorithms must be faster, more efficient, and less } \\
\text { complex: attempts can be made to study ameliorative strategies } \\
\text { in the future. }\end{array}$ & [357] \\
\hline ML & $\begin{array}{l}\text { Traffic forecasting, thanks to big data, ML, and network KPIs, can } \\
\text { estimate accurate statistical traffic characteristics of different types } \\
\text { of cells over both short- and long-term forecasts. }\end{array}$ & $\begin{array}{l}\text { The model makes use of a few critical network KPIs, yet it is } \\
\text { effective and does not affect the model's cost during training and } \\
\text { forecasting. }\end{array}$ & {$[358,359]$} \\
\hline ML & $\begin{array}{l}\text { Found a substantial association between cell load and the user } \\
\text { quality of a cell. It can be used to identify and implement interfer- } \\
\text { ence pairings using ML approaches. }\end{array}$ & $\begin{array}{l}\text { More powerful More data analytics methods are being researched } \\
\text { to solve the issues of } 4.9 \mathrm{G} \text { and } 5 \mathrm{G} \text { mobile network optimization. }\end{array}$ & [360-362] \\
\hline ML & $\begin{array}{l}\text { Used proactive caching techniques to collect large amounts of } \\
\text { existing data and use ML to ascertain content popularity. }\end{array}$ & $\begin{array}{l}\text { Cache location at base stations is also essential in the design of } \\
\text { ML tools. }\end{array}$ & [363-365] \\
\hline ML & $\begin{array}{l}\text { Documented a powerful framework for 5G network slicing and } \\
\text { the architecture of } 5 \mathrm{G} \text {. }\end{array}$ & $\begin{array}{l}\text { Edge computing, CORD applications, and SDN-based E-CORD, } \\
\text { RCORD, and M-CORD must be given priority. }\end{array}$ & [366-368] \\
\hline ML & $\begin{array}{l}\text { 5G-oriented cyberdefense architecture to swiftly and efficiently } \\
\text { identify cyberthreats in } 5 \mathrm{G} \text { mobile networks. }\end{array}$ & $\begin{array}{l}\text { To detection and classification to help establish which DL models } \\
\text { from a set and the optimal hyperparameters are more appropriate } \\
\text { for each configuration and throughput need. }\end{array}$ & {$[369,370]$} \\
\hline DL & $\begin{array}{l}\text { Estimate a power delay profile of a sub- } 6 \mathrm{GHz} \text { channel, which is } \\
\text { an input to the DNN. }\end{array}$ & $\begin{array}{l}\text { Developing multi-user beam selection, perform a comparative } \\
\text { investigation of other DL-based beam selection algorithms as well. }\end{array}$ & [374] \\
\hline 4- & Security & & \\
\hline DL & $\begin{array}{l}\text { Huge antenna arrays as well as millimetre Wave antenna arrays } \\
\text { are being utilised. }\end{array}$ & Solid set of coding algorithms is available. & [371-373] \\
\hline
\end{tabular}

\section{Discussion}

This research investigates the most important studies in state-of-the-art 5G and 6G networks that make use of IoT, energy and machine learning technologies. The purpose of this analysis is to draw attention to certain research tendencies in this field. This research is not up to date, and it would not address implementation, only the literature on the subject matter. This study differs from prior estimates in several ways. It is advised that the surrounding material be used as a taxonomy. Creating a literature taxonomy in a research 
subject may provide a number of advantages, some of which are evolving. On the one hand, the taxonomy of literary works is widely disseminated, while on the other, it is hardly discussed. The results of the survey revealed three aspects of the content of the literature, namely, challenges in the successful utilisation of these applications, recommendations to alleviate these difficulties, and the motivations for a general framework for the search and browse procedure that was proposed.

\subsection{Challenges}

$6 \mathrm{G}$ will be built on a set of technology enablers that will allow it to meet the demanding requirements of these new services. The following are the primary thrusts that we foresee (see Figure 6).

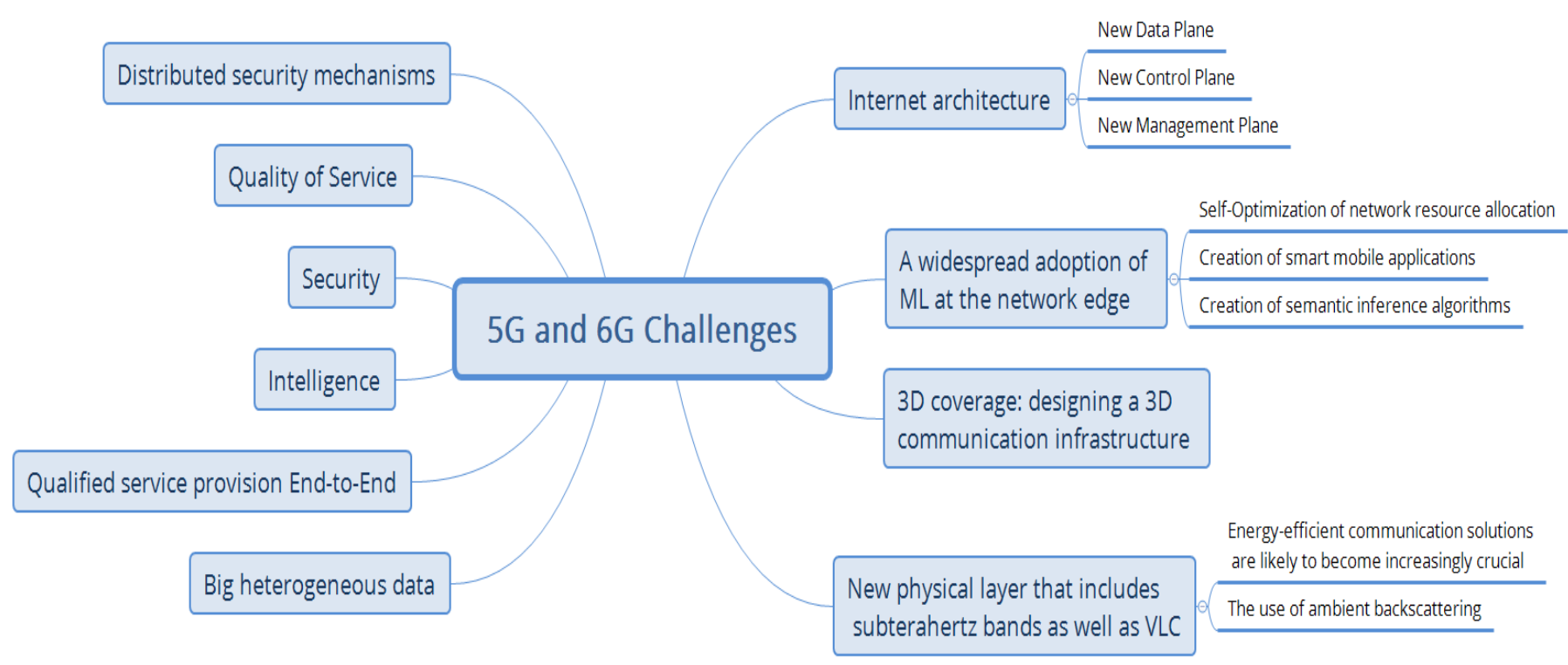

Figure 6. 5G and 6G Challenges.

\subsubsection{A New Internet Architecture}

The need to support nearly deterministic services, such as in high-precision manufacturing, while also ensuring very tight physical constraints such as latency and energy consumption, necessitates a new Internet architecture that brings together different resources, such as communication and computation, into a single framework.

(1) a new data plane that can adapt to different operating modes dynamically and support holographic communications. (2) a new control plane that allows for the synchronisation of concurrent streams for holographic communications, as well as the use of preferred path routing protocols to provide almost deterministic networks for high-precision manufacturing. (3) a new management plane that incorporates self-configuration and self-optimization capabilities and takes advantage of machine learning's strong support.

\subsubsection{Widespread Adoption of Machine Learning at the Network Edge}

Distributed machine learning algorithms, which may operate under severe time restrictions, are likely to play a vital role in a number of ways:

(1) self-optimization of network resource allocation, with proactive solutions based on network learning and prediction being considered. (2) creation of smart mobile applications that learn from user behaviour and operate as a context-aware virtual intelligent assistant, running either directly on mobile devices or remotely via computation-offloading techniques. (3) creation of semantic inference algorithms and semantic communication strategies to integrate knowledge representation into communication. This will be very helpful in deploying holographic communications effectively. 


\subsubsection{D Coverage}

By designing a 3D communication infrastructure that includes terrestrial and aerial radio access points as well as mobile edge hosts, cloud functions can be provided on demand.

This system is far more cost-effective than the existing approach, which relies on a fixed infrastructure, when requests fluctuate greatly over place and time, as they do with periodic events that draw large crowds or in remote areas, such as in the case of disasters. The idea is to manage a variety of aerial platforms, such as unmanned aerial vehicles, high-altitude (approximately $20 \mathrm{~km}$ ) platform stations, and constellations of Very-LowEarth-Orbit satellites flying at a few hundred kilometres in space, in order to provide cloud functionalities while maintaining controllable delay constraints.

\subsubsection{A New Physical Layer That includes Subterahertz Bands as Well as VLC}

The use of sub terahertz bands and VLC is required to support very high data speeds (as much as terabits per second) to enable, for example, holographic communications.

(1) Energy-efficient communication solutions are likely to become increasingly crucial, especially as the Internet of Things (IoT) becomes more widely deployed, with a plethora of tiny sensors. Ref. [388] discusses energy-harvesting mechanisms, sophisticated wireless charging methods, and their fundamental limits, with a focus on potential distributed laser charging approaches that demonstrate wireless charging can transfer up to $2 \mathrm{~W}$ of power over a distance of about $10 \mathrm{~m}$. (2) The use of ambient backscattering, which allows tiny devices to run without a battery by redirecting ambient radio-frequency (RF) signals without requiring active RF transmission [389], will be an even more radical approach.

\subsubsection{Distributed Security Mechanisms}

The concept so far outlined envisions a massive exchange of data to enable widespread adoption of machine learning techniques. Clearly, this poses a significant problem in terms of security, privacy, and trust, which 6G networks must address appropriately. To establish an effective compromise between ML and privacy, innovative cryptographic techniques should be applied. A mobile user, for example, may utilise homomorphic encryption [390] to execute a distant ML algorithm on its data. Instead of transmitting raw data, the user may send encrypted data, run the remote ML algorithm on the encrypted data, and still get the desired result. Existing techniques are now impractical due to their enormous computational complexity, but we can expect this to change in the next decade or two. Another important issue, particularly in the IoT situation, is decentralised authentication. Using blockchain-like methods, distributed ledger technologies are likely to play a crucial role in distributed authentication [391].

\subsubsection{Quality of Service}

In contrast to 5G, 6G's QoS will include additional metrics. Latency, throughput, and packet loss rate are some of the most used QoS KPIs. Because intelligence will become the primary necessity in the $6 \mathrm{G}$ era, compute capacity and storage will also need to be considered. Furthermore, rather than needing outstanding performance in a single area, future 6G systems will focus on the optimization of numerous measures, for which the complexity of determining the relationship exceeds the capabilities of current mathematical models. To overcome this issue, a variety of purpose-based learning models should be widely used.

\subsubsection{Security}

Since the Internet has become an increasingly significant part of people's lives and work, security has gotten a lot of attention. Despite the fact that DL has proved its effectiveness in threat detection [392], future communication services will necessitate context-aware security protection using a variety of data created in people's lives and work. Furthermore, when new strategies are developed to maintain message security, the choice 
of security configuration will become an even more difficult problem, as security protection usually entails some loss of network QoS.

\subsubsection{Intelligence}

Because future cellular networks will be more dynamic, system knowledge of potential congestion and environmental changes will be necessary to provide qualified services, with three obstacles. To begin, the communication system necessitates the ability to federate learning in order to meet the changing service requirements of mobile users in a timely manner. Second, automated and unexpected system upgrades are essential for adapting to new conditions. Third, in order to fully realise the potential of machine learning, the difficulties of heterogeneity in software and hardware must be addressed.

\subsubsection{Qualified Service Provision End-to-End}

Computation Oriented Communications (COC), Con-textually Agile eMBB Communications (CAeC), and Event Defined uRLLC (EDuRLLC) [393] are three types of newly supported 6G services, similar to 5G. Despite the fact that the exact service types enabled by $6 \mathrm{G}$ have yet to be determined, the standards will undoubtedly be more strict. For starters, future 6G services would require end-to-end assurances, which means the metric should be measured from the transceiver to the receiver rather than just the main network element in the $5 \mathrm{G}$ system. Second, in addition to typical measurements like link bandwidth, latency, and security, other KPIs should be considered. Future services will be evaluated in terms of situational awareness, learning capability, storage cost, and computing capacity. Furthermore, the heterogeneity of communication technologies and infrastructure hardware, as well as the complex requirements on numerous metrics, would hasten the adoption of machine learning in networking.

\subsubsection{Big Heterogeneous Data}

There are many various types of big data sources, each with its data rate, mobility, and packet loss. In wireless networks, analysing diverse data is difficult. Spatial and temporal dynamics are brought about by heterogeneous data. As a result, for massive spatiotemporal data processing in mobile networks, unusual methodologies are necessary.

\subsection{Recommendations}

This section gives some recommendations for addressing the concerns and challenges that have arisen in 6G through the application of machine learning techniques (see Figure 7).

When two primary tracks come together, a new generation is born: (1) a technological path that brings new, ground-breaking technologies to maturity; and (2) a societal path that encourages the introduction of new services which cannot be efficiently provided by current technology. We begin by introducing new services and then highlighting some of the primary enablers of those services.

\subsubsection{Holographic Communications}

will become obsolete within ten years. New modes of engagement emerge that allow for a real immersion into faraway worlds, displacing the current means of remote contact among human beings. In conjunction with holographic communications, it is envisaged that 5D communications and services, which integrate all human sense information (sight, hearing, touch, smell, and taste), will emerge, resulting in a totally immersive experience [394]. In order to implement holographic communications using multiple-view cameras, data rates on the order of terabits per second will be required [395], which will be insufficient for $5 \mathrm{G}$. 


\subsubsection{Manufacturing with High Precision}

One of the most important goals of Industry 4.0 is to eliminate the need for human intervention in industrial processes through the use of automatic control systems and communications technology. For high-precision manufacturing, this results in extremely high dependability (on the order of 1-109) and extremely low latency (0.1-1 ms round-trip time) [396]. Aside from that, industrial control networks demand real-time data transfer and excellent determinism, which translates into extremely low delay jitter, on the order of $1 \mathrm{~ns}$, which is translated into extremely low delay jitter.

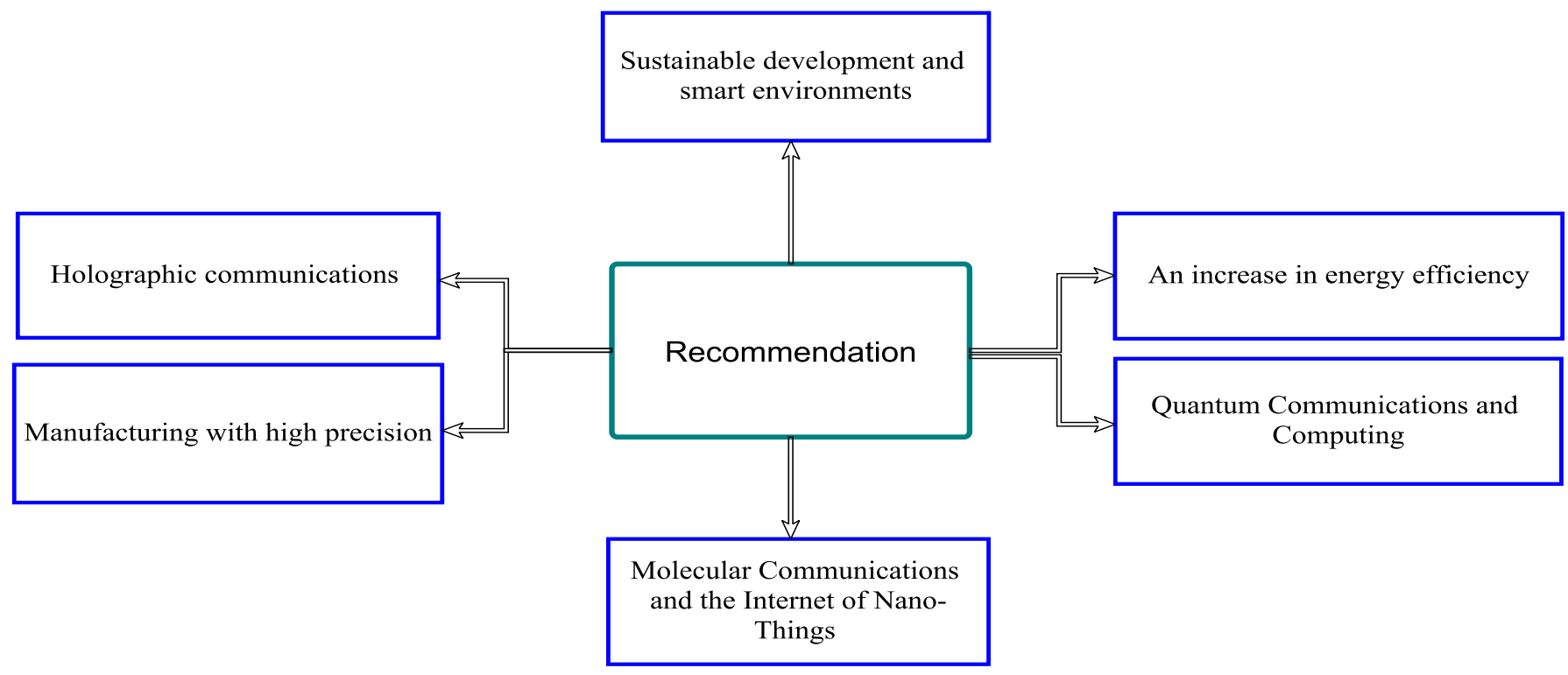

Figure 7. Categories of Motivations.

\subsubsection{Sustainable Development and Smart Environments}

Information and communications technologies (ICTs) incorporating wireless communications, cloud computing, and the Internet of Things (IoT) are expected to play a critical role in advancing global sustainability and improving the quality of life for all people. These technologies have the potential to make significant contributions to improving health care and enabling the creation of smart cities, which may include the construction of intelligent transportation and energy distribution infrastructure. A pervasive sensing framework, as well as a distributed decision and actuation system, are required to achieve some of these objectives. 6G will make a substantial contribution to this by depending on $3 \mathrm{D}$ communication platforms that can enable almost instantaneous distributed edge cloud features, such as distributed decision procedures, to be implemented. When it comes to situations such as autonomous driving, reliable safety procedures are critical in reducing the likelihood of an accident. This will necessitate extremely high levels of communication reliability (i.e., greater than $99.9999 \%$ ) as well as low end-to-end latency (below $1 \mathrm{~ms}$ ). Furthermore, intercommunication among automobiles will be critical in lowering the likelihood of a collision. To do this, high-speed data linkages between vehicles and between vehicles and roadside equipment will be required.

\subsubsection{An Increase in Energy Efficiency}

It goes without saying that any sustainable growth must pay strict attention to energy use. As a result, 6G will need to design communication tactics that are both effective and energy-efficient. In order to provide battery-free communication whenever possible, the goal is to achieve communication efficiency on the order of $1 \mathrm{pJ} / \mathrm{b}$. Some key performance indicators (KPIs), such as delay jitter and energy per bit, are not stated in 5G since they do not represent a primary focus of 5G, although they are critical KPIs for 6G. 


\subsubsection{Quantum Communications and Computing}

With the support of entire applications/scenarios, 6G will meet higher security needs than current technologies. With the application of a quantum key based on the quantum no-cloning theorem and the uncertainty principle, quantum communications can give extremely robust security [397]. Whenever eavesdroppers attempt to carry out observations, measurements, or copy activities in quantum communications, the quantum state is disrupted, and the eavesdropping activity may be easily identified. Theoretically, quantum communications have the potential to provide complete security. While $\mathrm{Tb} / \mathrm{s}$ data transmission and full applications/scenarios provide obstacles for wireless computing in 6G, they also present opportunities [397]. When compared to traditional computing, which uses $0-1-b$ operations, quantum computing, which relies on quantum superposition and entanglement, can significantly increase computing capabilities by utilising unitary transformations in the form of qubits. As a result, quantum computing has the potential to greatly accelerate and improve artificial intelligence algorithms that require large amounts of data and extensive training. Furthermore, by combining quantum theory and machine learning, it is possible to construct more powerful and efficient machine learning algorithms to meet the requirements of $6 \mathrm{G}$.

\subsubsection{Molecular Communications and the Internet of Nano-Things (Molecular} Communications and the Internet of Nano-Things)

Advanced nanotechnology has the potential to enable the production of nanodevices such as nano-robots, implantable chips, and biosensors, which have vital applications in areas such as nanoscale sensing and biomedicine [398]. The use of nanotechnology in biomedicine, in particular, has piqued interest because it has the potential to accomplish tasks such as intelligent drug distribution through blood vessels and monitoring of body organs, both of which have the potential to enhance significantly human healthcare outcomes. It is possible to achieve effective communication and information transmission by connecting nanodevices to the Internet or by forming networks (i.e., the Internet of Nano-Things); in biomedicine, the Internet of Bio-Nano-Things (IoBNT) can enable the connection of nanodevices and biological entities. In the Internet of Things, molecular communication is an enabling approach for the IoBNT, in which biological molecules are used to communicate and transport information amongst nanodevices [398]. A further benefit of combining the IoBNT with body area networks, which are short-distance wireless networks made up of wearable monitoring devices/sensors and sensing devices embedded in or on the body, is that they can give comprehensive solutions for healthcare enhancements.

\subsection{Motivations}

The future 6G network will not only concentrate on the pure communication area, but it will also be interoperable between diverse but related fields like electronics and materials, wireless communication, computer science and engineering, and computer science and technology [399]. Nanoelectronics for the Internet of Things, RF modules and packaging, high-frequency materials, radio transceivers, energy harvesting, THz imaging, and 2D/3D imaging will all be covered in the topic of electronics and materials. While in the field of computer science and engineering, the professionals can make contributions in a variety of areas such as image and signal analysis, mobile apps, security and privacy, big data analysis, smart sensor analytics, smart environments, and ubiquitous systems to name a few. In addition to their work in the field of wireless communication, experts in RF and antennas, 5G baseband technology, Internet of Things applications, future radio access, network optimization and management, spectrum regulations, and channel modelling are also actively involved in a variety of other fields (see Figure 8). 


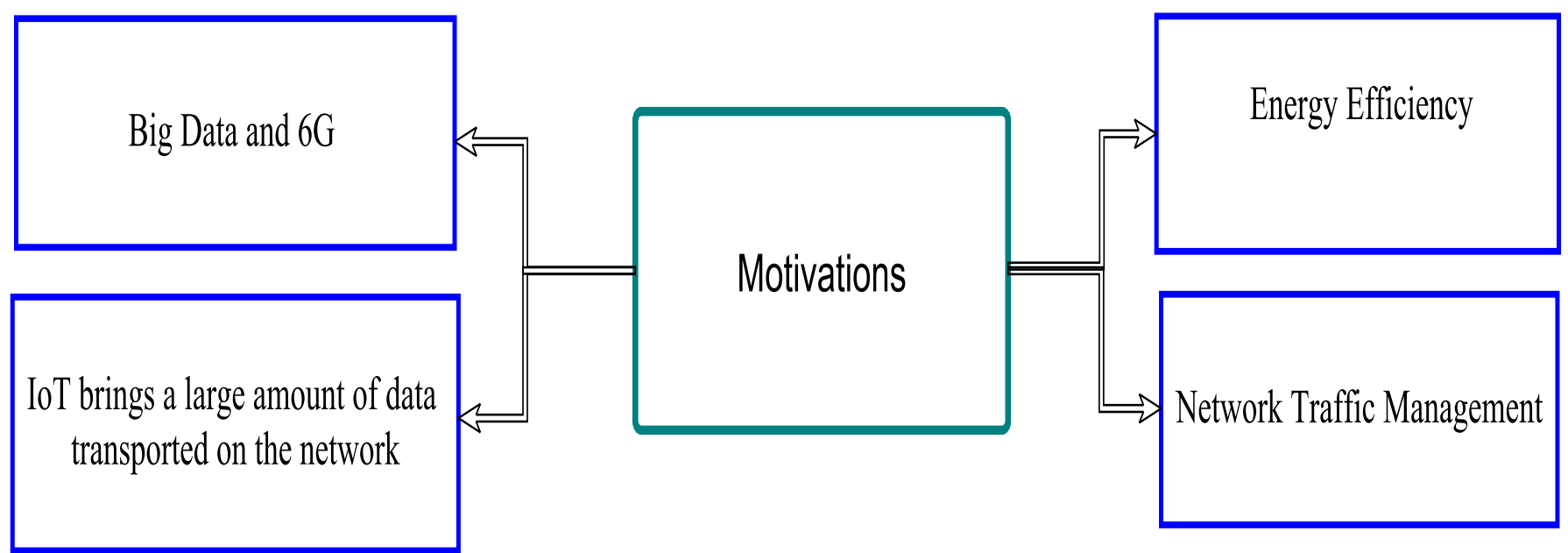

Figure 8. Categories of recommendations according to audience.

\subsubsection{Big Data and 6G}

In this section, we'll take a quick look at some of the outstanding questions. This also provides guidance for future advancements in research at the intersection of big data and $6 \mathrm{G}$, as tackling these difficulties has the potential to provide significant breakthroughs.

Caching and computing on a proactive basis: By utilising modern big data analytics methodologies, the cost of computing and caching for future generation networks can be decreased. As a result, resources are to be efficiently allocated and utilised, which will result in a better balance between caching and computing overhead. If the intermediate and final outcomes are not significant, they should not be stored because storing all of the information would be prohibitively expensive.

Security and privacy: Big data analytics is used to unearth information that has been hidden within a large amount of data. Because of this, large-scale data analysis may result in security and privacy concerns. In order to ensure that data cannot be modified or altered during the storage, management, and processing stages, it is critical that information is properly encrypted. Furthermore, only authorised entities should be permitted access to the data, and access should be granted only through secure means. As a result, security and privacy problems are important considerations for such large-scale data analysis, and they should be addressed thoughtfully.

Big Heterogeneous data: Large amounts of heterogeneous data: Large amounts of data from a variety of sources with varying data rates, mobility, and packet loss. Analysis of heterogeneous data in wireless networks is a difficult problem to solve. Spatial-temporal dynamics are brought about by heterogeneous data. As a result, for large-scale spatiotemporal data analysis in mobile networks, novel methodologies are necessary that are not commonly used.

\subsubsection{The Internet of Things (IoT)}

Carries with it a vast volume of data that must be carried via the network. As a result of the addition of sophisticated sensors and controllers to their devices, several device manufacturers had already included the capacity of collecting data from their gadgets. Within this decade, it became obvious that reporting on and analysing data gathered from sensors and control devices were becoming increasingly popular among people.

Management of a smart city. In a smart city, information is available on a variety of topics, including the environment, tourism, traffic, social life, mobility, energy, and so on. A complete picture of the city is provided by the data collection and organisation provided by heterogeneous sensors, which allows for the implementation of early warning solutions and the development of predictive models, thereby increasing the city's resilience 
and limiting the negative effects on citizens caused by unexpected events. The environmental monitoring and structural monitoring applications are the ones that stand out in this scenario.

Management of public and private metering systems. To make the development of smart metering systems economically viable, the goal in this scenario is to integrate smart metering services into a single transportation network. For example, the citizen or company can remotely monitor the status of their house or office, allowing the system to adapt quickly to various occurrences in order to provide the desired comfort while conserving energy, as seen in the image below. In this context, data privacy and security are critical considerations: it is vital to ensure that the information gathered will not be used to violate the privacy of the user or be utilised by malevolent parties in order to do this.

Management of industrial processes as part of a whole. The requirement for integrated industrial management is a crucial component of innovation processes in the Fourth Industrial Revolution (Industrie 4.0). The establishment of dedicated network infrastructure, on the other hand, can be a considerable financial burden. It is anticipated that the $5 \mathrm{G}$ network would provide an infrastructure that will meet the demanding specifications required for industrial automation applications while still being cost-effective. In this scenario, the ability to meet the severe URLLC requirements will be critical in determining the outcome.

\subsubsection{Energy Efficiency}

Widespread adoption of cloud computing and network functions virtualization (NFV), combined with a smart, programmable management system, will result in a significant reduction in service deployment time, significant savings in energy consumption, and improved network management in general [400]. Cloud computing and NFV, combined with a smart, programmable management system, will enable a significant reduction in service deployment time, significant savings in energy consumption, and improved network management in general [401]. In this scenario, ML can be utilised to improve performance by making greater use of available network and virtual machine resources while also reducing total energy requirements and expenses. The nature of NFV opens the door to more flexible management allowing for fast, dynamic deployment to match current demand in real-time, as well as the migration of services to locations with reduced energy costs and/or footprints [401,402], When used in conjunction with a smart management system, this can result in downscaling tactics that can result in significant savings in both energy consumption and operating expenses[403-405].

\subsubsection{Network Traffic Management}

Accurate network traffic identification is the foundation of intelligent network management, and it is essential for effective network management. Service providers will be unable to maximise shared resource consumption and assure accurate billing and payment if they do not identify and measure network traffic flow. On the basis of past traffic data, machine learning algorithms can be used to find the most optimum network topologies. Similarly, network functions virtualization (NFV) can be used to provide the necessary resources and services to overcome exceptional or routine situations. Because of this, researchers in communication networks are increasingly relying on machine learning to optimise network architecture, control, and administration, ultimately leading to greater automation in network operations. Experts in the machine learning field, on the other hand, are collaborating with networking researchers to optimise network architecture and design. Furthermore, the rapid growth of machine learning has had an impact on many fields of wireless communication since it is capable of making judgments and extracting information from the data generated by a $5 \mathrm{G}$ network. 


\title{
9. Conclusions
}

Recent research on sixth-generation wireless communication networks especially concerning IoT applications, machine learning, and energy has been reviewed in this paper. Future breakthroughs in these domains are expected to significantly impact many aspects of our life over the next several years, regardless of industry. The next-generation networks will likely be highly complicated. They will require energy that can power billions of connected devices simultaneously, making it impractical to change or recharge the batteries of so many devices regularly. As a result, energy-efficient networks and devices will be required in the future. Because of the importance of energy efficiency in future 6G networks, this paper provided a survey of recent works in 5G and 6G energy efficiency for IoT networks. This paper also reviewed the primary challenges and limitations that must be addressed to achieve energy efficiency-based huge IoT enabled by 6G. This study aims to put forward a set of guidelines to researchers working in energy efficiency, IoT, and AI for the next-generation mobile communication networks.

Author Contributions: Conceptualization, M.N.M., M.M. and A.R.A.; methodology, M.N.M. and A.R.A.; validation, M.N.M. and H.N. and A.R.A.; formal analysis, H.N. and M.A.S.; investigation, M.N.M., M.M. and Q.S.Q.; resources, M.N.M., Q.S.Q. and A.R.A.; data curation, M.N.M., H.N., M.M. and A.R.A.; writing-original draft preparation, M.N.M., M.M. and A.R.A.; writing-review and editing, M.N.M., H.N., and A.R.A.; visualization, M.N.M., M.M. and H.N.; supervision, M.N.M. and Q.S.Q.; project administration, A.R.A.; funding acquisition, A.R.A. All authors have read and agreed to the published version of the manuscript.

Funding: Universiti Tenaga Nasional (UNITEN), Innovation and Research Management Center (iRMC): RJO10517844/005 (2020).

Institutional Review Board Statement: Not applicable.

Data Availability Statement: Not applicable.

Acknowledgments: This research was sponsored and supported under the Universiti Tenaga Nasional (UNITEN) internal grant no RJO10517844/005 (2020). Many thanks to the Innovation and Research Management Center (iRMC), UNITEN who provided their assistance and expertise during the research.

Conflicts of Interest: The authors declare no conflict of interest.

\author{
Abbreviations \\ The following abbreviations are used in this manuscript: \\ 3GPP Third Generation Partnership Project \\ mMTC Massive Machine Type Communications \\ AI Artificial Intelligence \\ mURLLC Massive Ultrareliable Low Latency Communication \\ AR Augmented reality \\ ML Machine learning \\ B5G Beyond 5G \\ $\mathrm{m} 2 \mathrm{~m} \quad$ machine to machine \\ BL Bayesian learning \\ MR Mixed reality \\ CU Cellular mode \\ NB Naïve Bayes \\ EDoS Economic Denial of Sustainability \\ NR New Radio \\ E2E end to end \\ NFV Network function virtualization \\ eMBB Enhanced Mobile Broadband \\ NMA Network management automation \\ EHD Extremely high definition
}




$\begin{array}{ll}\text { NN } & \text { Neural networks } \\ \text { eTOM } & \text { Enhanced Telecom Operations Map } \\ \text { XR } & \text { Extended reality } \\ \text { FiWi } & \text { Fiber Wireless } \\ \text { xMBB } & \text { Extreme mobile broadband } \\ \text { FL } & \text { Fuzzy logic } \\ \text { RF } & \text { Random forests } \\ \text { GPT } & \text { General purpose technology } \\ \text { RA } & \text { Resource allocation } \\ \text { HCSs } & \text { Human Centric Services } \\ \text { RL } & \text { Reinforcement learning } \\ \text { DR } & \text { Deep learning } \\ \text { RNN } & \text { Recurrent Neural Networks } \\ \text { DT } & \text { Decision trees } \\ \text { SG } & \text { Smart grid } \\ \text { D2D } & \text { Device-to-device } \\ \text { SINR } & \text { Signal to interference plus noise ratio Service } \\ \text { DRL } & \text { Deep reinforcement learning } \\ \text { SLAs } & \text { Level Agreements } \\ \text { ITSs } & \text { Intelligent transportation systems } \\ \text { SHD } & \text { Super high definition } \\ \text { IoE } & \text { Internet of Everything } \\ \text { SGs } & \text { Smart grids } \\ \text { IoT } & \text { Internet of Things } \\ \text { SRS } & \text { Shopping recommender system } \\ \text { ITS } & \text { Intelligent transportation system } \\ \text { SON } & \text { Self organizing networks } \\ \text { IMD } & \text { Intelligent medical diagnosis } \\ \text { SVM } & \text { Support Vector Machine } \\ \text { LTE } & \text { Long Term Evolution } \\ \text { THz } & \text { Terahertz } \\ \text { QC } & \text { Quantum computing } \\ \text { TL } & \text { Transfer learning } \\ \text { QML } & \text { Quantum ML } \\ \text { UAVs } & \text { Unmanned aerial vehicles } \\ \text { QoE } & \text { Quality of experience } \\ \text { URLLC } & \text { Ultra reliable low latency communication } \\ \text { QoS } & \text { Quality of service } \\ \text { VNF } & \text { Virtual network function } \\ \text { KPIs } & \text { Key performance Indicators } \\ \text { VR } & \text { Virtual reality } \\ \text { MIMO } & \text { Multiple Input Multiple Output } \\ \text { Hyper high speed railway } \\ \text { massive Internet of Things } \\ \text { HOT } & \end{array}$

\section{References}

1. Pouttu, A.; Burkhardt, F.; Patachia, C.; Mendes, L.; Brazil, G.R.; Pirttikangas, S.; Jou, E.; Kuvaja, P.; Heikkilä, M.; Päivärinta, T.; et al. 6G White Paper on Validation and Trials for Verticals towards 2030's; 6G Research Visions. 2020. Available online: https: //www.6gchannel.com/items/6g-white-paper-validation-trials/ (accessed on 15 July 2021).

2. Series, M. IMT Vision-Framework and overall objectives of the future development of IMT for 2020 and beyond. Recomm. ITU Geneva 2015, 2083, 1-21.

3. Castelli, M.; Vanneschi, L.; Popovič, A. Predicting burned areas of forest fires: An artificial intelligence approach. Fire Ecol. 2015, 11, 106-118. [CrossRef]

4. Qi, Q.; Chen, X.; Zhong, C.; Zhang, Z. Integration of energy, computation and communication in 6G cellular internet of things. IEEE Commun. Lett. 2020, 24, 1333-1337. [CrossRef]

5. Amgoune, H.; Mazri, T. 5G: Interconnection of Services and Security Approaches. In Proceedings of the 3rd International Conference on Smart City Applications, New York, NY, USA, 2-4 October 2018; pp. 1-7. 
6. Vidal, J.M.; Monge, M.A.S.; Villalba, L.J.G. Detecting Workload-based and Instantiation-based Economic Denial of Sustainability on 5G environments. In Proceedings of the 13th International Conference on Availability, Reliability and Security, Hamburg, Germany, 27-30 August 2018; pp. 1-8.

7. Vreman, N.; Maggio, M. Multilayer distributed control over 5G networks: Challenges and security threats. In Proceedings of the Workshop on Fog Computing and the IoT, New York, NY, USA, 15 April 2019; pp. 31-35.

8. Zhang, S.; Zhu, D. Towards artificial intelligence enabled 6G: State of the art, challenges, and opportunities. Comput. Netw. 2020, 183, 107556. [CrossRef]

9. Sun, Y.; Liu, J.; Wang, J.; Cao, Y.; Kato, N. When machine learning meets privacy in 6g: A survey. IEEE Commun. Surv. Tutor. 2020, 22, 2694-2724. [CrossRef]

10. Sheth, K.; Patel, K.; Shah, H.; Tanwar, S.; Gupta, R.; Kumar, N. A taxonomy of AI techniques for 6G communication networks. Comput. Commun. 2020, 161, 279-303. [CrossRef]

11. Wang, M.; Zhu, T.; Zhang, T.; Zhang, J.; Yu, S.; Zhou, W. Security and privacy in 6G networks: New areas and new challenges. Digit. Commun. Netw. 2020, 6, 281-291. [CrossRef]

12. Sekaran, R.; Patan, R.; Raveendran, A.; Al-Turjman, F.; Ramachandran, M.; Mostarda, L. Survival Study on Blockchain Based 6G-Enabled Mobile Edge Computation for IoT Automation. IEEE Access 2020, 8, 143453-143463. [CrossRef]

13. Akhtar, M.W.; Hassan, S.A.; Ghaffar, R.; Jung, H.; Garg, S.; Hossain, M.S. The shift to 6G communications: Vision and requirements. Hum.-Centric Comput. Inf. Sci. 2020, 10, 1-27. [CrossRef]

14. Hewa, T.; Gür, G.; Kalla, A.; Ylianttila, M.; Bracken, A.; Liyanage, M. The role of blockchain in 6G: Challenges, opportunities and research directions. In Proceedings of the 2020 2nd 6G Wireless Summit (6G SUMMIT), Levi, Finland, 17-20 March 2020; pp. 1-5.

15. Alam, I.; Sharif, K.; Li, F.; Latif, Z.; Karim, M.; Biswas, S.; Nour, B.; Wang, Y. A survey of network virtualization techniques for internet of things using sdn and nfv. ACM Comput. Surv. (CSUR) 2020, 53, 1-40. [CrossRef]

16. Davids, C.; Gurbani, V.K.; Ormazabal, G.; Rollins, A.; Singh, K.; State, R. Research topics related to real-time communications over 5G networks. ACM SIGCOMM Comput. Commun. Rev. 2018, 46, 1-6. [CrossRef]

17. Jun, S.-H.; Kim, J.-H. 5G will popularize virtual and augmented reality: KT's trials for world's first 5G olympics in Pyeongchang. In Proceedings of the International Conference on Electronic Commerce, Turku, Finland, 14-16 June 2017; pp. 1-8.

18. Cherrared, S.; Imadali, S.; Fabre, E.; Gössler, G.; Yahia, I.G.B. A survey of fault management in network virtualization environments: Challenges and solutions. IEEE Trans. Netw. Serv. Manag. 2019, 16, 1537-1551. [CrossRef]

19. Fourati, H.; Maaloul, R.; Chaari, L. Self-organizing cellular network approaches applied to 5G networks. In Proceedings of the 2019 Global Information Infrastructure and Networking Symposium (GIIS), Tunis, Tunisia, 18-20 December 2019; pp. 1-4.

20. Klaine, P.V.; Imran, M.A.; Onireti, O.; Souza, R.D. A survey of machine learning techniques applied to self-organizing cellular networks. IEEE Commun. Surv. Tutor. 2017, 19, 2392-2431. [CrossRef]

21. Gebremariam, A.A.; Usman, M.; Qaraqe, M. Applications of Artificial Intelligence and Machine Learning in the Area of SDN and NFV: A Survey. In Proceedings of the 2019 16th International Multi-Conference on Systems, Signals \& Devices (SSD), Istanbul, Turkey, 21-24 March 2019; pp. 545-549.

22. Buda, T.S.; Assem, H.; Xu, L.; Raz, D.; Margolin, U.; Rosensweig, E.; Lopez, D.; Corici, J.; Smirnov, M.; Mullins, R.; et al. Can machine learning aid in delivering new use cases and scenarios in 5G? In Proceedings of the NOMS 2016-2016 IEEE/IFIP Network Operations and Management Symposium, Istanbul, Turkey, 25-29 April 2016; pp. 1279-1284.

23. Mwanje, S.; Decarreau, G.; Mannweiler, C.; Naseer-ul-Islam, M.; Schmelz, L.C. Network management automation in 5G: Challenges and opportunities. In Proceedings of the 2016 IEEE 27th Annual International Symposium on Personal, Indoor, and Mobile Radio Communications (PIMRC), Valencia, Spain, 4-8 September 2016; pp. 1-6.

24. Tham, M.-L.; Iqbal, A.; Chang, Y.C. Deep Reinforcement Learning for Resource Allocation in 5G Communications. In Proceedings of the 2019 Asia-Pacific Signal and Information Processing Association Annual Summit and Conference (APSIPA ASC), Lanzhou, China, 18-21 November 2019; pp. 1852-1855.

25. Lee, Y.L.; Qin, D. A survey on applications of deep reinforcement learning in resource management for 5G heterogeneous networks. In Proceedings of the 2019 Asia-Pacific Signal and Information Processing Association Annual Summit and Conference (APSIPA ASC), Lanzhou, China, 18-21 November 2019; pp. 1856-1862.

26. Sultan, K.; Ali, H. Where big data meets 5G? In Proceedings of the Second International Conference on Internet of things, Data and Cloud Computing, Cambridge, UK, 22-23 March 2017; pp. 1-4.

27. Aldossari, S.M.; Chen, K.-C. Machine learning for wireless communication channel modeling: An overview. Wirel. Pers. Commun. 2019, 106, 41-70. [CrossRef]

28. Tudzarov, A.; Gelev, S. 5G and software network paradigm. In Proceedings of the 2018 23rd International Scientific-Professional Conference on Information Technology (IT), Žabljak, Montenegro, 19-24 February 2018; pp. 1-5.

29. Moysen, J.; Giupponi, L. From 4G to 5G: Self-organized network management meets machine learning. Comput. Commun. 2018, 129, 248-268. [CrossRef]

30. Cayamcela, M.E.M.; Lim, W. Artificial intelligence in 5G technology: A survey. In Proceedings of the 2018 International Conference on Information and Communication Technology Convergence (ICTC), Jeju Island, Korea, 17-19 October 2018; pp. 860-865. 
31. Dormidontova, E.; Komarov, M. Quality of Services Provided for Users in Fifth Generation Wireless Networks. In Proceedings of the 8th International Conference on Information Communication and Management, Shenyang, China, 21-23 September 2018; pp. 32-38.

32. Mulvey, D.; Foh, C.H.; Imran, M.A.; Tafazolli, R. Cell fault management using machine learning techniques. IEEE Access 2019, 7, 124514-124539. [CrossRef]

33. Marquez, C.; Gramaglia, M.; Fiore, M.; Banchs, A.; Costa-Perez, X. How should I slice my network? A multi-service empirical evaluation of resource sharing efficiency. In Proceedings of the 24th Annual International Conference on Mobile Computing and Networking, Shenyang, China, 21-23 September 2018; pp. 191-206.

34. Amgoune, H.; Mazri, T. Comparison between different 5G architectures for a better integration of these services and proposal of an improved architecture. In Proceedings of the 4th International Conference on Smart City Applications, Casablanca, Morocco, 2-4 October 2019; pp. 1-7.

35. Albreem, M.A.; Juntti, M.; Shahabuddin, S. Massive MIMO detection techniques: A survey. IEEE Commun. Surv. Tutor. 2019, 21, 3109-3132. [CrossRef]

36. Mucchi, L.; Jayousi, S.; Caputo, S.; Paoletti, E.; Zoppi, P.; Geli, S.; Dioniso, P. How 6 G technology can change the future wireless healthcare. In Proceedings of the 2020 2nd 6G wireless summit (6G SUMMIT), Levi, Finland, 17-20 March 2020; pp. 1-6.

37. Nizzi, F.; Pecorella, T.; Bastianini, M.; Cerboni, C.; Buzzigoli, A.; Fratini, A. The role of network simulator in the 5G experimentation. In Proceedings of the 2019 Workshop on Next-Generation Wireless with ns-3, Florence, Italy, 21 June 2019 ; pp. 13-17.

38. Dahlman, E.; Parkvall, S.; Peisa, J.; Tullberg, H. 5G evolution and beyond. In Proceedings of the 2019 IEEE 20th International Workshop on Signal Processing Advances in Wireless Communications (SPAWC), Cannes, France, 2-5 July 2019; pp. 1-5.

39. Arjoune, Y.; Faruque, S. Artificial Intelligence for 5G Wireless Systems: Opportunities, Challenges, and Future Research Direction. In Proceedings of the 2020 10th Annual Computing and Communication Workshop and Conference (CCWC), Las Vegas, NV, USA, 6-8 January 2020; pp. 1023-1028.

40. Jansevskis, M.; Osis, K. Machine Learning and on 5G Based Technologies Create New Opportunities to Gain Knowledge. In Proceedings of the 2018 2nd European Conference on Electrical Engineering and Computer Science (EECS), Bern, Switzerland, 20-22 December 2018; pp. 376-381.

41. Morocho-Cayamcela, M.E.; Lee, H.; Lim, W. Machine learning for 5G/B5G mobile and wireless communications: Potential, limitations, and future directions. IEEE Access 2019, 7, 137184-137206. [CrossRef]

42. Zhang, C.; Patras, P.; Haddadi, H. Deep learning in mobile and wireless networking: A survey. IEEE Commun. Surv. Tutor. 2019, 21, 2224-2287. [CrossRef]

43. Ahmed, R.; Matin, M.A. Towards 6G wireless networks-challenges and potential technologies. J. Electr. Eng. 2020, $71,290-297$.

44. Dogra, A.; Jha, R.K.; Jain, S. A survey on beyond 5G network with the advent of 6G: Architecture and emerging technologies. IEEE Access 2020, 9, 67512-67547. [CrossRef]

45. Alsharif, M.H.; Kelechi, A.H.; Albreem, M.A.; Chaudhry, S.A.; Zia, M.S.; Kim, S. Sixth generation (6G) wireless networks: Vision, research activities, challenges and potential solutions. Symmetry 2020, 12, 676. [CrossRef]

46. Hajiyat, Z.R.; Ismail, A.; Sali, A.; Hamidon, M.N. Antenna in 6G wireless communication system: Specifications, challenges, and research directions. Optik 2021, 231, 166415. [CrossRef]

47. Aggarwal, S.; Kumar, N.; Tanwar, S. Blockchain Envisioned UAV Communication Using 6G Networks: Open issues, Use Cases, and Future Directions. IEEE Internet Things J. 2020. [CrossRef]

48. Salh, A.; Audah, L.; Shah, N.S.M.; Alhammadi, A.; Abdullah, Q.; Kim, Y.H.; Al-Gailani, S.A.; Hamzah, S.A.; Esmail, B.A.F.; Almohammedi, A.A. A Survey on Deep Learning for Ultra-Reliable and Low-Latency Communications Challenges on 6G Wireless Systems. IEEE Access 2021, 9, 55098-55131. [CrossRef]

49. Piran, M.J.; Suh, D.Y. Learning-driven wireless communications, towards 6G. In Proceedings of the 2019 International Conference on Computing, Electronics \& Communications Engineering (iCCECE), London, UK, 22-23 August 2019; pp. $219-224$.

50. Wikström, G.; Peisa, J.; Rugeland, P.; Johansson, N.; Parkvall, S.; Girnyk, M.; Mildh, G.; Da Silva, I. Challenges and technologies for 6G. In Proceedings of the 2020 2nd 6G Wireless Summit (6G SUMMIT), Levi, Finland, 17-20 March 2020; pp. 1-5.

51. Mourad, A.; Yang, R.; Lehne, P.H.; de la Oliva, A. Towards 6G: Evolution of key performance indicators and technology trends. In Proceedings of the 2020 2nd 6G Wireless Summit (6G SUMMIT), Levi, Finland, 17-20 March 2020; pp. 1-5.

52. Ajmal, S.; Muzammil, M.B.; Jamil, A.; Abbas, S.M.; Iqbal, U.; Touseef, P. Survey on cache schemes in heterogeneous networks using $5 \mathrm{~g}$ internet of things. In Proceedings of the 3rd International Conference on Future Networks and Distributed Systems, Paris, France, 1-2 July 2019; pp. 1-8.

53. Lu, Z.; Huang, Y.-C.; Bangjun, C. A Study for Application in Vehicle Networking and Driverless Driving. In Proceedings of the 2019 3rd International Conference on Computer Science and Artificial Intelligence, Normal, IL, USA, 6-8 December 2019; pp. 264-267.

54. Tal, I.; Muntean, G.-M. Towards reasoning vehicles: A survey of fuzzy logic-based solutions in vehicular networks. ACM Comput. Surv. (CSUR) 2017, 50, 1-37. [CrossRef]

55. Rahmani, R.; Kanter, T. Autonomous cooperative decision-making in massively distributed IoT via heterogenous networks. In Proceedings of the 1st International Conference on Internet of Things and Machine Learning, Liverpool, UK, 17-18 October 2017; pp. 1-5. 
56. Muhamedyev, R.I.; Kalimoldaev, M.N.; Uskenbayeva, R.K. Semantic network of ICT domains and applications. In Proceedings of the 2014 Conference on Electronic Governance and Open Society: Challenges in Eurasia, St. Petersburg, Russian, 18-20 November 2014; pp. 178-186.

57. Nakamura, Y.; Shimonishi, H.; Kobayashi, Y.; Satoda, K.; Matsunaga, Y.; Kanetomo, D. Novel heterogeneous computing platforms and 5G communications for IoT applications. In Proceedings of the 2017 IEEE/ACM International Conference on Computer-Aided Design (ICCAD), Irvine, CA, USA, 13-16 November 2017; pp. 874-879.

58. Suryanegara, M.; Arifin, A.S.; Asvial, M. The IoT-based transition strategy towards 5G. In Proceedings of the International Conference on Big Data and Internet of Thing, London, UK, 20-22 December 2017; pp. 186-190.

59. Ding, J.; Nemati, M.; Ranaweera, C.; Choi, J. IoT connectivity technologies and applications: A survey. arXiv 2020, arXiv:2002.12646.

60. Bithas, P.S.; Michailidis, E.T.; Nomikos, N.; Vouyioukas, D.; Kanatas, A.G. A survey on machine-learning techniques for UAV-based communications. Sensors 2019, 19, 5170. [CrossRef]

61. Usman, M.; Asghar, M.R.; Granelli, F.; Qaraqe, K. Integrating smart city applications in 5G networks. In Proceedings of the 2nd International Conference on Future Networks and Distributed Systems, Amman, Jordan, 26-27 June 2018; pp. 1-5.

62. Ullah, Z.; Al-Turjman, F.; Mostarda, L.; Gagliardi, R. Applications of artificial intelligence and machine learning in smart cities. Comput. Commun. 2020, 154, 313-323. [CrossRef]

63. Rao, S.K.; Prasad, R. Impact of 5G technologies on industry 4.0. Wirel. Pers. Commun. 2018, 100, 145-159. [CrossRef]

64. Velev, D.; Zlateva, P.; Zong, X. Challenges of 5G usability in disaster management. In Proceedings of the 2018 International Conference on Computing and Artificial Intelligence, Chengdu, China, 12-14 March 2018; pp. 71-75.

65. Muhamedyev, R.I.; Amirgaliyev, Y.N.; Kalimoldayev, M.N.; Khamitov, A.N.; Abdilmanova, A. Selection of the most prominent lines of research in ICT domain. In Proceedings of the 2015 Twelve International Conference on Electronics Computer and Computation (ICECCO), Almaty, Kazakhstan, 27-30 September 2015; pp. 1-7.

66. Kim, J.H. 6G and Internet of Things: A survey. J. Manag. Anal. 2021, 8, 316-332.

67. Shafique, K.; Khawaja, B.A.; Sabir, F.; Qazi, S.; Mustaqim, M. Internet of things (IoT) for next-generation smart systems: A review of current challenges, future trends and prospects for emerging 5G-IoT scenarios. IEEE Access 2020, 8, 23022-23040. [CrossRef]

68. Sharma, S.K.; Wang, X. Towards massive machine type communications in ultra-dense cellular IoT networks: Current issues and machine learning-assisted solutions. IEEE Commun. Surv. Tutor. 2019. [CrossRef]

69. Malik, U.M.; Javed, M.A.; Zeadally, S.; ul Islam, S. Energy efficient fog computing for 6G enabled massive IoT: Recent trends and future opportunities. IEEE Internet Things J. 2021. [CrossRef]

70. Nawaz, S.J.; Sharma, S.K.; Wyne, S.; Patwary, M.N.; Asaduzzaman, M. Quantum machine learning for 6G communication networks: State-of-the-art and vision for the future. IEEE Access 2019, 7, 46317-46350. [CrossRef]

71. Usama, M.; Erol-Kantarci, M. A survey on recent trends and open issues in energy efficiency of 5G. Sensors 2019, $19,3126$. [CrossRef] [PubMed]

72. Huo, Y.; Dong, X.; Xu, W.; Yuen, M. Enabling multi-functional 5G and beyond user equipment: A survey and tutorial. IEEE Access 2019, 7, 116975-117008. [CrossRef]

73. Temesgene, D.A.; Núñez-Martínez, J.; Dini, P. Softwarization and optimization for sustainable future mobile networks: A survey. IEEE Access 2017, 5, 25421-25436. [CrossRef]

74. Sultan, K.; Ali, H.; Zhang, Z. Big data perspective and challenges in next generation networks. Future Internet 2018, 10, 56. [CrossRef]

75. Imoize, A.L.; Adedeji, O.; Tandiya, N.; Shetty, S. 6G Enabled Smart Infrastructure for Sustainable Society: Opportunities, Challenges, and Research Roadmap. Sensors 2021, 21, 1709. [CrossRef] [PubMed]

76. Mahmood, N.H.; Alves, H.; López, O.A.; Shehab, M.; Osorio, D.P.M.; Latva-Aho, M. Six key features of machine type communication in 6G. In Proceedings of the 2020 2nd 6G Wireless Summit (6G SUMMIT), Levi, Finland, 17-20 March 2020; pp. $1-5$.

77. Xu, C.; Ishikawa, N.; Rajashekar, R.; Sugiura, S.; Maunder, R.G.; Wang, Z.; Yang, L.; Hanzo, L. Sixty years of coherent versus non-coherent tradeoffs and the road from $5 \mathrm{G}$ to wireless futures. IEEE Access 2019, 7, 178246-178299. [CrossRef]

78. Wang, M.; Lin, Y.; Tian, Q.; Si, G. Transfer Learning Promotes 6G Wireless Communications: Recent Advances and Future Challenges. IEEE Trans. Reliab. 2021. [CrossRef]

79. Zayas, A.D.; Rico, D.; García, B.; Merino, P. A coordination framework for experimentation in $5 \mathrm{~g}$ testbeds: Urllc as use case. In Proceedings of the 17th ACM International Symposium on Mobility Management and Wireless Access, Miami Beach, FL, USA, 25-29 November 2019; pp. 71-79.

80. Nikaein, N.; Marina, M.K.; Manickam, S.; Dawson, A.; Knopp, R.; Bonnet, C. OpenAirInterface: A flexible platform for 5G research. ACM SIGCOMM Comput. Commun. Rev. 2014, 44, 33-38. [CrossRef]

81. Herculea, D.; Chen, C.S.; Haddad, M.; Capdevielle, V. Straight: Stochastic geometry and user history based mobility estimation. In Proceedings of the 8th ACM International Workshop on Hot Topics in Planet-Scale mObile Computing and Online Social neTworking, Paderborn, Germany, 5-8 July 2016; pp. 1-6.

82. Mishra, P.K.; Pandey, S. A Method for Mode Selection in a dynamic network for Device-to-Device Communication for 5G. In Proceedings of the International Conference on Informatics and Analytics, Pondicherry, India, 25-26 August 2016; pp. 1-6. 
83. Huang, X.; Zhao, Z.; Zhang, H. Latency analysis of cooperative caching with multicast for 5G wireless networks. In Proceedings of the 2016 IEEE/ACM 9th International Conference on Utility and Cloud Computing (UCC), Shanghai, China, 6-9 December 2016; pp. 316-320.

84. Panwar, G.; Tourani, R.; Mick, T.; Mtibaa, A.; Misra, S. DICE: Dynamic multi-RAT selection in the ICN-enabled wireless edge. In Proceedings of the Workshop on Mobility in the Evolving Internet Architecture, Los Angeles CA, USA, 25 August 2017; pp. 31-36.

85. Ateya, A.A.; Muthanna, A.; Gudkova, I.; Vybornova, A.; Koucheryavy, A. Intelligent core network for Tactile Internet system. In Proceedings of the International Conference on Future Networks and Distributed Systems, Cambridge, UK, 19-20 July 2017; pp. 1-6.

86. Polese, M.; Mezzavilla, M.; Rangan, S.; Zorzi, M. Mobility management for TCP in mmWave networks. In Proceedings of the 1st ACM Workshop on Millimeter-Wave Networks and Sensing Systems 2017, Snowbird, UT, USA 16 October 2017; pp. 11-16.

87. Seraoui, Y.; Belmekki, M.; Bellafkih, M.; Raouyane, B. eTOM mapping onto NFV framework: IMS use case. In Proceedings of the 2nd International Conference on Computing and Wireless Communication Systems, Larache Morocco, 14-16 November 2017; pp. $1-8$.

88. Liu, Y.-N.; Wang, X.-W.; Li, J.; Huang, M. A Wireless Spectrum Management Mechanism Based on the Theory of Double Auction in Cognitive Cellular Networks. In Proceedings of the 2017 International Conference on Wireless Communications, Networking and Applications, Shenzhen, China, 20-22 October 2017; pp. 16-23.

89. Tranoris, C.; Denazis, S.; Guardalben, L.; Pereira, J.; Sargento, S. Enabling Cyber-Physical Systems for 5G networking: A case study on the Automotive Vertical domain. In Proceedings of the 2018 IEEE/ACM 4th International Workshop on Software Engineering for Smart Cyber-Physical Systems (SEsCPS), Gothenburg, Sweden, 27 May-3 June 2018; pp. 37-40.

90. Bojovic, B.; Lagen, S.; Giupponi, L. Implementation and evaluation of frequency division multiplexing of numerologies for 5G new radio in ns-3. In Proceedings of the 10th Workshop on ns-3, Surathkal, India, 13-14 June 2018; pp. 37-44.

91. Zugno, T.; Polese, M.; Zorzi, M. Integration of carrier aggregation and dual connectivity for the ns-3 mmWave module. In Proceedings of the 10th Workshop on ns-3, Surathkal, India, 13-14 June 2018; pp. 45-52.

92. Mukherjee, S.; Ravindran, R.; Raychaudhuri, D. A distributed core network architecture for 5G systems and beyond. In Proceedings of the 2018 Workshop on Networking for Emerging Applications and Technologies, Budapest, Hungary, 20 August 2018; pp. 33-38.

93. Guija, D.; Siddiqui, M.S. Identity and access control for micro-services based 5G NFV platforms. In Proceedings of the 13th International Conference on Availability, Reliability and Security, Hamburg, Germany, 27-30 August 2018; pp. 1-10.

94. Rosa, R.V.; Rothenberg, C.E. Blockchain-based decentralized applications meet multi-administrative domain networking. In Proceedings of the ACM SIGCOMM 2018 Conference on Posters and Demos, Budapest Hungary, 20-25 August 2018; pp. 114-116.

95. de Oliveira, G.R.; Storck, C.R.; Duarte-Figueiredo, F. SoftH: Soft handover multicriteria mechanism. In Proceedings of the 34th ACM/SIGAPP Symposium on Applied Computing, Limassol, Cyprus, 8-12 April 2019; pp. 2422-2429.

96. Kassem, M.M.; Marina, M.K.; Radunovic, B. DIY model for mobile network deployment: A step towards 5G for all. In Proceedings of the 1st ACM SIGCAS Conference on Computing and Sustainable Societies, Menlo Park and San Jose, CA, USA, 20-22 June 2018; pp. 1-5.

97. Mesodiakaki, A.; Zola, E.; Kassler, A. Joint user association and backhaul routing for green 5G mesh millimeter wave backhaul networks. In Proceedings of the 20th ACM International Conference on Modelling, Analysis and Simulation of Wireless and Mobile Systems, Miami, FL, USA, 21-25 November 2017; pp. 179-186.

98. Lv, Z.; Wang, Z.; Lv, Y.; Yuan, M. An AirSea Manta-Ray Robot in 5G OGCE. In Proceedings of the 2019 2nd International Conference on Service Robotics Technologies, Beijing, China, 22-24 March 2019; pp. 63-67.

99. Raca, D.; Leahy, D.; Sreenan, C.J.; Quinlan, J.J. Beyond throughput, the next generation: A 5G dataset with channel and context metrics. In Proceedings of the 11th ACM Multimedia Systems Conference, Istanbul, Turkey, 8-11 June 2020; pp. 303-308.

100. Aloqaily, M.; Balasubramanian, V.; Zaman, F.; Ridhawi, I.A.; Jararweh, Y. Congestion mitigation in densely crowded environments for augmenting qos in vehicular clouds. In Proceedings of the 8th ACM Symposium on Design and Analysis of Intelligent Vehicular Networks and Applications, Montreal, QC, Canada, 28 October-2 November 2018; pp. 49-56.

101. Makris, N.; Passas, V.; Korakis, T.; Tassiulas, L. Employing MEC in the Cloud-RAN: An Experimental Analysis. In Proceedings of the 2018 on Technologies for the Wireless Edge Workshop, New Delhi, India, 2 November 2018; pp. 15-19.

102. Patané, G.M.; Valastro, G.C.; Sambo, Y.A.; Ozturk, M.; Hussain, S.; Imran, M.A.; Panno, D. Flexible SDN/NFV-based SON testbed for 5G mobile networks. In Proceedings of the 2019 IEEE/ACM 23rd International Symposium on Distributed Simulation and Real Time Applications (DS-RT), Cosenza, Italy, 7-9 October 2019; pp. 1-8.

103. Subramanya, T.; Riggio, R.; Rasheed, T. Intent-based mobile backhauling for 5G networks. In Proceedings of the 201612 th International Conference on Network and Service Management (CNSM), Montreal, QC, Canada, 31 October-4 November 2016; pp. 348-352.

104. Park, K.H.; Kim, J.S.; Cha, S.H.; Chung, M.Y. System Capacity Analysis of Millimeter Wave Band with Polarized Antenna Structure. In Proceedings of the 12th International Conference on Ubiquitous Information Management and Communication, Langkawi, Malaysia, 5-7 January 2018; pp. 1-6. 
105. Lundgren, C.; Skoogh, A.; Johansson, B.; Stahre, J.; Friis, M. The value of 5G connectivity for maintenance in manufacturing industry. In Proceedings of the 2017 Winter Simulation Conference (WSC), Las Vegas, NV, USA, 3-6 December 2017; pp. 3964-3975.

106. Beyranvand, H.; Lévesque, M.; Maier, M.; Salehi, J.A.; Verikoukis, C.; Tipper, D. Toward 5G: FiWi enhanced LTE-A HetNets with reliable low-latency fiber backhaul sharing and WiFi offloading. IEEE/ACM Trans. Netw. 2016, 25, 690-707. [CrossRef]

107. Chirivella-Perez, E.; Calero, J.M.A.; Wang, Q.; Gutiérrez-Aguado, J. Towards a realistic 5G infrastructure emulator for experimental service deployment and performance evaluation. In Proceedings of the 2018 IEEE/ACM 22nd International Symposium on Distributed Simulation and Real Time Applications (DS-RT), Madrid, Spain, 15-17 October 2018; pp. 1-7.

108. Kantor, M.; State, R.; Engel, T.; Ormazabal, G. A policy-based per-flow mobility management system design. In Proceedings of the Principles, Systems and Applications on IP Telecommunications, Chicago, IL, USA, 6-8 October 2015; pp. 35-42.

109. Ginzboorg, P.; Niemi, V. Privacy of the long-term identities in cellular networks. In Proceedings of the 9th EAI International Conference on Mobile Multimedia Communications, Xi'an, China, 18-20 June 2016; pp. 167-175.

110. Qaddus, A.; Aslam, S.; Minhas, A.A.; Almazyad, A. 5G Hetrogeneous Cellular Networks a Viable Mode for Implementing Future Smart Grid Networks. In Proceedings of the International Conference on Future Networks and Distributed Systems, Cambridge, UK, 19-20 July 2017.

111. Hussain, S.R.; Echeverria, M.; Singla, A.; Chowdhury, O.; Bertino, E. Insecure connection bootstrapping in cellular networks: The root of all evil. In Proceedings of the 12th Conference on Security and Privacy in Wireless and Mobile Networks, Miami, FL, USA, 15-17 May 2019; pp. 1-11.

112. Blanc, G.; Kheir, N.; Ayed, D.; Lefebvre, V.; de Oca, E.M.; Bisson, P. Towards a 5G security architecture: Articulating softwaredefined security and security as a service. In Proceedings of the 13th International Conference on Availability, Reliability and Security, Hamburg, Germany, 27-30 August 2018; pp. 1-8.

113. Annessi, R.; Fabini, J.; Zseby, T. To Trust or Not to Trust: Data Origin Authentication for Group Communication in $5 \mathrm{G}$ Networks. In Proceedings of the 13th International Conference on Availability, Reliability and Security, Hamburg, Germany, 27-30 August 2018; pp. 1-7.

114. Adam, I.; Ping, J. Framework for security event management in 5G. In Proceedings of the 13th International Conference on Availability, Reliability and Security, Hamburg, Germany, 27-30 August 2018; pp. 1-7.

115. Hanane, O.; Tomader, M. 4G and 5G: Security and privacy analysis. In Proceedings of the 4th International Conference on Big Data and Internet of Things, Rabat, Morocco, 23-24 October 2019; pp. 1-4.

116. Basin, D.; Dreier, J.; Hirschi, L.; Radomirovic, S.; Sasse, R.; Stettler, V. A formal analysis of 5 G authentication. In Proceedings of the 2018 ACM SIGSAC Conference on Computer and Communications Security, Toronto, ON, Canada, 15-19 October 2018; pp. 1383-1396.

117. Ma, T.; Hu, F.; Ma, M. Securing 5G HetNets Using Mutual Physical Layer Authentication. In Proceedings of the 2019 7th International Conference on Information Technology: IoT and Smart City, Shanghai, China, 20-23 December 2019; pp. 275-278.

118. Cho, J.Y.; Sergeev, A.; Zou, J. Securing ethernet-based optical fronthaul for $5 \mathrm{~g}$ network. In Proceedings of the 14 th International Conference on Availability, Reliability and Security, Canterbury, UK, 26-29 August 2019; pp. 1-6.

119. Mangiante, S.; Klas, G.; Navon, A.; GuanHua, Z.; Ran, J.; Silva, M.D. Vr is on the edge: How to deliver 360 videos in mobile networks. In Proceedings of the Workshop on Virtual Reality and Augmented Reality Network, Los Angeles, CA, USA, 25 August 2017; pp. 30-35.

120. Chakareski, J. VR/AR immersive communication: Caching, edge computing, and transmission trade-offs. In Proceedings of the Workshop on Virtual Reality and Augmented Reality Network, Los Angeles, CA, USA, 25 August 2017; pp. 36-41.

121. Reale, A.; Tóth, M.; Horváth, Z. Towards context aware computations offloading in 5G. In Proceedings of the 11th European Conference on Software Architecture: Companion Proceedings, Canterbury, UK, 11-15 September 2017; pp. 89-92.

122. Sun, L.; Duanmu, F.; Liu, Y.; Wang, Y.; Ye, Y.; Shi, H.; Dai, D. Multi-path multi-tier 360-degree video streaming in $5 \mathrm{G}$ networks. In Proceedings of the 9th ACM Multimedia Systems Conference, Amsterdam, The Netherlands, 12-15 June 2018; pp. 162-173.

123. Bujari, A.; Palazzi, C.E.; Perale, D. Addressing bandwidth demand in full-immersive virtual reality. In Proceedings of the 5th EAI International Conference on Smart Objects and Technologies for Social Good, Valencia, Spain, 25-27 September 2019; pp. 13-18.

124. Foukas, X.; Marina, M.K.; Kontovasilis, K. Orion: RAN slicing for a flexible and cost-effective multi-service mobile network architecture. In Proceedings of the 23rd Annual International Conference on Mobile Computing and Networking, Snowbird, UT, USA, 16-20 October 2017; pp. 127-140.

125. Kim, D.H.; Kazmi, S.A.; Hong, C.S. Cooperative slice allocation for virtualized wireless network: A matching game approach. In Proceedings of the 12th International Conference on Ubiquitous Information Management and Communication, Langkawi, Malaysia, 5-7 January 2018; pp. 1-6.

126. Challa, R.; Jeon, S.; Raza, S.M.; Thorat, P.; Choo, H. Superflex: Network slicing based super flexible 5 G architecture. In Proceedings of the 12th International Conference on Ubiquitous Information Management and Communication, Langkawi, Malaysia, 5-7 January 2018; pp. 1-4.

127. Gramaglia, M.; Pavón, I.L.; Gringoli, F.; Garcia-Aviles, G.; Serrano, P. Design and Validation of a Multi-service 5G Network with QoE-aware Orchestration. In Proceedings of the 12th International Workshop on Wireless Network Testbeds, Experimental Evaluation \& Characterization, New Delhi, India, 2 November 2018; pp. 11-18. 
128. Nikaein, N.; Schiller, E.; Favraud, R.; Katsalis, K.; Stavropoulos, D.; Alyafawi, I.; Zhao, Z.; Braun, T.; Korakis, T. Network store: Exploring slicing in future 5G networks. In Proceedings of the 10th International Workshop on Mobility in the Evolving Internet Architecture, Paris, France, 7 September 2015; pp. 8-13.

129. Sathi, V.N.; Srinivasan, M.; Thiruvasagam, P.K.; Chebiyyam, S.R.M. A novel protocol for securing network slice component association and slice isolation in 5G networks. In Proceedings of the 21st ACM International Conference on Modeling, Analysis and Simulation of Wireless and Mobile Systems, Montreal, QC, Canada, 28 October-2 November 2018; pp. $249-253$.

130. Oliveira, A.; Vazão, T. Adapting priority schemes to achieve network slice isolation. In Proceedings of the 35th Annual ACM Symposium on Applied Computing, Brno, Czech Republic, 30 March-3 April 2020; pp. 1164-1171.

131. Martiradonna, S.; Abrardo, A.; Moretti, M.; Piro, G.; Boggia, G. Architecting RAN slicing for URLLC: Design decisions and open issues. In Proceedings of the 2019 IEEE/ACM 23rd International Symposium on Distributed Simulation and Real Time Applications (DS-RT), Cosenza, Italy, 7-9 October 2019; pp. 1-4.

132. Nikaein, N.; Chang, C.-Y.; Alexandris, K. Mosaic5G: Agile and flexible service platforms for 5G research. ACM SIGCOMM Comput. Commun. Rev. 2018, 48, 29-34. [CrossRef]

133. Akundi, S.; Prabhu, S.; K, N.U.B.; Mondal, S.C. Suppressing Noisy Neighbours in 5G networks: An end-to-end NFV-based framework to detect and suppress noisy neighbours. In Proceedings of the 21st International Conference on Distributed Computing and Networking, Kolkata, India, 4-7 January 2020; pp. 1-6.

134. Xue, J.; Shao, H.; Ma, Q. Resource allocation for system throughput maximization based on mobile edge computing. In Proceedings of the 2018 International Conference on Electronics and Electrical Engineering Technology, Tianjin, China, 19-21 September 2018; pp. 177-181.

135. Ferdouse, L.; Ejaz, W.; Anpalagan, A.; Khattak, A.M. Joint workload scheduling and BBU allocation in cloud-RAN for 5G networks. In Proceedings of the Symposium on Applied Computing, Marrakech, Morocco, 3-7 April 2017; pp. 621-627.

136. Mathur, R.P.; Pratap, A.; Misra, R. Distributed algorithm for resource allocation in uplink 5G networks. In Proceedings of the 7th ACM International Workshop on Mobility, Interference, and MiddleWare Management in HetNets, Chennai, India, 10-14 July 2017; pp. 1-6.

137. Cao, T.; Xu, C.; Wang, M.; Jiang, Z.; Chen, X.; Zhong, L.; Grieco, L. Stochastic optimization for green multimedia services in dense 5G networks. ACM Trans. Multimed. Comput. Commun. Appl. (TOMM) 2019, 15, 1-22. [CrossRef]

138. Dogo, E.; Salami, A.; Nwulu, N. Evaluative analysis of next generation mobile networks in future smart grid in developing countries. In Proceedings of the 4th International Conference on Smart City Applications, Casablanca, Morocco, 2-4 October 2019; pp. 1-10.

139. Hsiung, T.; Kanza, Y. Simct: Spatial simulation of urban evolution to test resilience of $5 \mathrm{~g}$ cellular networks. In Proceedings of the 2nd ACM SIGSPATIAL International Workshop on GeoSpatial Simulation, Casablanca, Morocco, 2-4 October 2019; pp. 1-8.

140. Sun, P.; Naser, H. A Service Slicing Strategy with QoS for LTE-based Cellular Networks. In Proceedings of the 14th ACM International Symposium on QoS and Security for Wireless and Mobile Networks, Montreal, QC, Canada, 28 October-2 November 2018; pp. 63-69.

141. Rony, R.I.; Lopez-Aguilera, E.; Garcia-Villegas, E. Access-aware backhaul optimization in 5G. In Proceedings of the 16th ACM International Symposium on Mobility Management and Wireless Access, Montreal, QC, Canada, 28 October-2 November 2018; pp. 124-127.

142. Li, H.; Ren, H.; Wu, J. Domain specific compiler for coordinated signal processing in 5G testbed. In Proceedings of the Workshop on Smart Internet of Things, San Jose, CA, USA, 14 October 2017; pp. 1-5.

143. Barros, V.A.; Junior, S.A.; Bruschi, S.M.; Monaco, F.J.; Estrella, J.C. An IoT multi-protocol strategy for the interoperability of distinct communication protocols applied to web of things. In Proceedings of the 25th Brazillian Symposium on Multimedia and the Web, Rio de Janeiro, Brazil, 29 October-1 November 2019; pp. 81-88.

144. Cho, J.; der Merwe, J.V. Poster: A new scalable, programmable and evolvable mobile control plane platform. In Proceedings of the 23rd Annual International Conference on Mobile Computing and Networking, Snowbird, UT, USA, 16-20 October 2017; pp. 540-542.

145. Mathur, S.; Saha, D.; Raychaudhuri, D. Poster: Cross-layer MAC/PHY protocol to support IoT traffic in 5G. In Proceedings of the 22nd Annual International Conference on Mobile Computing and Networking, MobiCom 2016, New York, NY, USA, 3-7 October 2016; pp. 467-468.

146. Sagari, S.S.; Mathur, S.; Saha, D.; Amin, S.O.; Ravindran, R.; Seskar, I.; Raychaudhuri, D.; Wang, G. Realization of CDMA-based IoT services with shared band operation of LTE in 5G. In Proceedings of the Workshop on Mobile Edge Communications, Los Angeles CA, USA, 21 August 2017; pp. 37-42.

147. Ali, F; Yigang, H. Spectrum for Next Generation Technologies. In Proceedings of the 2019 8th International Conference on Software and Information Engineering, Cairo, Egypt, 9-12 April 2019; pp. 188-191.

148. Ruuskanen, J.; Peng, H.; Martins, A. Latency prediction in 5G for control with deadtime compensation. In Proceedings of the Workshop on Fog Computing and the IoT, Montreal, QC, Canada, 15 April 2019; pp. 51-55.

149. Tsai, C.; Moh, M. Cache management for $5 \mathrm{G}$ cloud radio access networks. In Proceedings of the 12 th International Conference on Ubiquitous Information Management and Communication, Langkawi, Malaysia, 5-7 January 2018; pp. 1-8.

150. Meroni, G.; Mizmizi, M.; Plebani, P.; Reggiani, L. Improving Mobile Business Process Monitoring with Enhanced NFV MANO; Association for Computing Machinery: Brno, Czech Republic, 2020. 
151. Su, G.; Moh, M. Improving energy efficiency and scalability for IoT communications in 5 G networks. In Proceedings of the 12 th International Conference on Ubiquitous Information Management and Communication, Langkawi, Malaysia, 5-7 January 2018; pp. 1-8.

152. Desikan, K.S.; Srinivasan, M.; Murthy, C.S.R. A novel distributed latency-aware data processing in fog computing-enabled iot networks. In Proceedings of the ACM Workshop on Distributed Information Processing in Wireless Networks, Chennai, India, 10-14 July 2017; pp. 1-6.

153. Choudhari, T.; Moh, M.; Moh, T.-S. Prioritized task scheduling in fog computing. In Proceedings of the ACMSE 2018 Conference, Richmond, Kentucky, 29-31 March 2018; pp. 1-8.

154. Pratap, A.; Concone, F.; Nadendla, V.S.S.; Das, S.K. Three-Dimensional Matching based Resource Provisioning for the Design of Low-Latency Heterogeneous IoT Networks. In Proceedings of the 22nd International ACM Conference on Modeling, Analysis and Simulation of Wireless and Mobile Systems, Miami Beach, FL, USA, 25-29 November 2019; pp. 79-86.

155. Kaur, G.; Moh, M. Cloud computing meets 5G networks: Efficient cache management in cloud radio access networks. In Proceedings of the ACMSE 2018 Conference, Richmond, Kentucky, 29-31 March 2018; pp. 1-8.

156. Roozbeh, A. Distributed cloud and de-centralized control plane: A proposal for scalable control plane for 5G. In Proceedings of the 2015 IEEE/ACM 8th International Conference on Utility and Cloud Computing (UCC), Limassol, Cyprus, 7-10 December 2015; pp. 348-353.

157. Aftab, M.U.; Munir, Y.; Oluwasanmi, A.; Qin, Z.; Aziz, M.H.; Son, N.T. A Hybrid Access Control Model With Dynamic COI for Secure Localization of Satellite and IoT-Based Vehicles. IEEE Access 2020, 8, 24196-24208. [CrossRef]

158. Najm, I.A.; Hamoud, A.K.; Lloret, J.; Bosch, I. Machine learning prediction approach to enhance congestion control in 5G IoT environment. Electronics 2019, 8, 607. [CrossRef]

159. Guerrero-Pérez, A.D. Resource Management of IoT Devices in Restrictive Environments. In Proceedings of the 1st ACM Workshop on Emerging Smart Technologies and Infrastructures for Smart Mobility and Sustainability, Los Cabos, Mexico, 21 October 2019; pp. 33-38.

160. Chahlaoui, F.; El-Fenni, M.R.; Dahmouni, H. Performance analysis of load balancing mechanisms in SDN networks. In Proceedings of the 2nd International Conference on Networking, Information Systems \& Security, Rabat, Morocco, 27-29 March 2019; pp. 1-8

161. Luntovskyy, A.; Globa, L. Performance, Reliability and Scalability for IoT. In Proceedings of the 2019 International Conference on Information and Digital Technologies (IDT), Zilina, Slovakia, 25-27 June 2019; pp. 316-321.

162. Vukobratovic, D.; Jakovetic, D.; Skachek, V.; Bajovic, D.; Sejdinovic, D.; Kurt, G.K.; Hollanti, C.; Fischer, I. CONDENSE: A reconfigurable knowledge acquisition architecture for future 5G IoT. IEEE Access 2016, 4, 3360-3378. [CrossRef]

163. Baniata, M.; Ji, H.; Kim, Y.; Choi, J.; Hong, J. Energy-balancing unequal concentric chain clustering (MIMO-UCC) protocol for IoT system in 5G environment. In Proceedings of the 2018 Conference on Research in Adaptive and Convergent Systems, Honolulu, HI, USA, 9-12 October 2018; pp. 68-74.

164. Ismail, D.; Rahman, M.; Saifullah, A. Low-power wide-area networks: Opportunities, challenges, and directions. In Proceedings of the Workshop Program of the 19th International Conference on Distributed Computing and Networking, Varanasi, India, 4-7 January 2018; pp. 1-6.

165. Jsm, L.M.; Lokesh, V.; Polyzos, G.C. Energy efficient context based forwarding strategy in named data networking of things. In Proceedings of the 3rd ACM Conference on Information-Centric Networking, Kyoto, Japan, 26-28 September 2016; pp. 249-254

166. Haider, D.; Yang, X.; Abbasi, Q.H. Post-surgical fall detection by exploiting the 5 G C-Band technology for eHealth paradigm. Appl. Soft Comput. 2019, 81, 105537. [CrossRef]

167. Liu, Y.; Yang, Y.; Han, P.; Shao, Z.; Li, C. Virtual network embedding in fiber-wireless access networks for resource-efficient iot service provisioning. IEEE Access 2019, 7, 65506-65517. [CrossRef]

168. Maier, M.; Ebrahimzadeh, A. Towards immersive Tactile Internet experiences: Low-latency FiWi enhanced mobile networks with edge intelligence. IEEE/OSA J. Opt. Commun. Netw. 2019, 11, B10-B25. [CrossRef]

169. Sharma, S.K.; Wang, X. Collaborative distributed Q-learning for RACH congestion minimization in cellular IoT networks. IEEE Commun. Lett. 2019, 23, 600-603. [CrossRef]

170. Sandoval, R.M.; Canovas-Carrasco, S.; Garcia-Sanchez, A.-J.; Garcia-Haro, J. Smart usage of multiple RAT in IoT-oriented 5G networks: A reinforcement learning approach. In Proceedings of the 2018 ITU Kaleidoscope: Machine Learning for a 5G Future (ITU K), Santa Fe, Argentina, 26-28 November 2018; pp. 1-8.

171. Loghin, D.; Cai, S.; Chen, G.; Dinh, T.T.A.; Fan, F.; Lin, Q.; Ng, J.; Chin Ooi, B.; Sun, X.; Ta, Q.T.; et al. The disruptions of 5G on data-driven technologies and applications. IEEE Trans. Knowl. Data Eng. 2020, 32, 1179-1198. [CrossRef]

172. Messaoud, S.; Bradai, A.; Moulay, E. Online GMM Clustering and Mini-Batch Gradient Descent Based Optimization for Industrial IoT 4.0. IEEE Trans. Ind. Inform. 2019, 16, 1427-1435. [CrossRef]

173. Vukobratovic, D.; Jakovetic, D.; Skachek, V.; Bajovic, D.; Sejdinovic, D. Network function computation as a service in future 5G machine type communications. In Proceedings of the 2016 th International Symposium on Turbo Codes and Iterative Information Processing (ISTC), Brest, France, 5-9 September 2016; pp. 365-369.

174. Din, S.; Ahmad, A.; Paul, A. Human enabled green IoT in 5G networks. In Proceedings of the Symposium on Applied Computing, Marrakech, Morocco, 3-7 April 2017; pp. 208-213.

175. Wang, W.; Mao, C.; Zhao, S.; Cao, Y.; Yi, Y.; Chen, S.; Liu, Q. A Smart Semipartitioned Real-Time Scheduling Strategy for Mixed-Criticality Systems in 6G-Based Edge Computing. Wirel. Commun. Mob. Comput. 2021, 2021, 6663199. 
176. Lin, K.; Li, Y.; Zhang, Q.; Fortino, G. AI-Driven Collaborative Resource Allocation for Task Execution in 6G-Enabled Massive IoT. IEEE Internet Things J. 2021, 8, 5264-5273. [CrossRef]

177. Dong, W.; Xu, Z.-H.; Li, X.-X.; Xiao, S.-P. Low-cost subarrayed sensor array design strategy for iot and future $6 \mathrm{~g}$ applications. IEEE Internet Things J. 2020, 7, 4816-4826. [CrossRef]

178. Yang, Z.; Fang, Y.; Han, G.; Huq, K.M.S. Spatially-Coupled Protograph LDPC-Coded Hierarchical Modulated BICM-ID Systems: A Promising Transmission Technique for 6G-Enabled Internet of Things. IEEE Internet Things J. 2020. [CrossRef]

179. Liu, R.W.; Nie, J.; Garg, S.; Xiong, Z.; Zhang, Y.; Hossain, M.S. Data-driven trajectory quality improvement for promoting intelligent vessel traffic services in 6G-enabled maritime IoT systems. IEEE Internet Things J. 2020. [CrossRef]

180. Khan, L.U.; Yaqoob, I.; Imran, M.; Han, Z.; Hong, C.S. 6G wireless systems: A vision, architectural elements and future directions. IEEE Access 2020, 8, 147029-147044. [CrossRef]

181. Liu, C.; Feng, W.; Chen, Y.; Wang, C.-X.; Ge, N. Cell-free satellite-UAV networks for 6G wide-area Internet of Things. IEEE J. Sel. Areas Commun. 2020. [CrossRef]

182. Mukherjee, A.; Goswami, P.; Khan, M.A.; Manman, L.; Yang, L.; Pillai, P. Energy Efficient Resource Allocation strategy in Massive IoT for Industrial 6G Applications. IEEE Internet Things J. 2020. [CrossRef]

183. Hazra, A.; Adhikari, M.; Amgoth, T.; Srirama, S.N. Stackelberg Game for Service Deployment of IoT-Enabled Applications in 6G-aware Fog Networks. IEEE Internet Things J. 2020. [CrossRef]

184. Jia, Z.; Sheng, M.; Li, J.; Zhou, D.; Han, Z. Joint HAP Access and LEO Satellite Backhaul in 6G: Matching Game based Approaches. IEEE J. Sel. Areas Commun. 2020. [CrossRef]

185. Wang, W.; Liu, F.; Zhi, X.; Zhang, T.; Huang, C. An Integrated Deep Learning Algorithm for Detecting Lung Nodules with Low-dose CT and Its Application in 6G-enabled Internet of Medical Things. IEEE Internet Things J. 2020. [CrossRef]

186. Mardini, W.; Aljawarneh, S.; Al-Abdi, A. Using Multiple RPL Instances to Enhance the Performance of New 6G and Internet of Everything (6G/IoE)-Based Healthcare Monitoring Systems. Mob. Netw. Appl. 2021, 26, 952-968. [CrossRef]

187. Qi, F.; Li, W.; Yu, P.; Feng, L.; Zhou, F. Deep learning-based BackCom multiple beamforming for 6G UAV IoT networks. EURASIP J. Wirel. Commun. Netw. 2021, 2021, 50. [CrossRef]

188. Wang, J.; Zhang, Y. Using cloud computing platform of $6 \mathrm{G} \mathrm{IoT} \mathrm{in} \mathrm{e-commerce} \mathrm{personalized} \mathrm{recommendation.} \mathrm{Int.} \mathrm{J.} \mathrm{Syst.} \mathrm{Assur.}$ Eng. Manag. 2021, 12, 654-666. [CrossRef]

189. Lin, J.C.-W.; Srivastava, G.; Zhang, Y.; Djenouri, Y.; Aloqaily, M. Privacy preserving multi-objective sanitization model in 6G IoT environments. IEEE Internet Things J. 2020. [CrossRef]

190. Liao, Z.; Peng, J.; Huang, J.; Wang, J.; Wang, J.; Sharma, P.K.; Ghosh, U. Distributed probabilistic offloading in edge computing for 6g-enabled massive internet of things. IEEE Internet Things J. 2021, 8, 5298-5308. [CrossRef]

191. Wang, F.; Zhu, M.; Wang, M.; Khosravi, M.R.; Ni, Q.; Yu, S.; Qi, L. 6G-enabled short-term forecasting for large-scale traffic flow in massive IoT based on time-aware Locality-Sensitive Hashing. IEEE Internet Things J. 2020. [CrossRef]

192. Gao, Y. Using artificial intelligence approach to design the product creative on 6G industrial internet of things. Int. J. Syst. Assur. Eng. Manag. 2021, 12, 696-704. [CrossRef]

193. Xu, L. Application of wearable devices in 6G internet of things communication environment using artificial intelligence. Int. J. Syst. Assur. Eng. Manag. 2021, 12, 741-747. [CrossRef]

194. Ye, Z.; Su, L. The use of data mining and artificial intelligence technology in art colors and graph and images of computer vision under 6G internet of things communication. Int. J. Syst. Assur. Eng. Manag. 2021, 12, 689-695. [CrossRef]

195. Han, G.; Tu, J.; Liu, L.; Martínez-García, M.; Peng, Y. Anomaly Detection Based on Multidimensional Data Processing for Protecting Vital Devices in 6G-Enabled Massive IIoT. IEEE Internet Things J. 2021, 8, 5219-5229. [CrossRef]

196. Li, Q.; Xiao, R. The use of data mining technology in agricultural e-commerce under the background of $6 \mathrm{G}$ Internet of things communication. Int. J. Syst. Assur. Eng. Manag. 2021, 12, 813-823. [CrossRef]

197. Chen, N.; Okada, M. Towards 6G Internet of Things and the Convergence with RoF System. IEEE Internet Things J. 2020. [CrossRef]

198. Manal, R.; Fatima, R.; Tomader, M. Authentication for e-health applications in IoT enabled 5G and proposed solution. In Proceedings of the 4th International Conference on Smart City Applications, Casablanca, Morocco, 2-4 October 2019; pp. 1-6.

199. Fatima, R.; Manal, R.; Tomader, M. Cryptography in e-Health using 5G based IOT: A comparison study. In Proceedings of the 4th International Conference on Big Data and Internet of Things, Rabat, Morocco, 23-24 October 2019; pp. 1-6.

200. Rahimi, H.; Zibaeenejad, A.; Rajabzadeh, P.; Safavi, A.A. On the security of the 5G-IoT architecture. In Proceedings of the International Conference on Smart Cities and Internet of Things, Mashhad, Iran, 26-27 September 2018; pp. 1-8.

201. Shaik, A.; Borgaonkar, R.; Park, S.; Seifert, J.-P. New vulnerabilities in 4G and 5G cellular access network protocols: Exposing device capabilities. In Proceedings of the 12th Conference on Security and Privacy in Wireless and Mobile Networks, Miami, FL, USA, 15-17 May 2019; pp. 221-231.

202. Zenger, C.T.; Zimmer, J.; Pietersz, M.; Driessen, B.; Paar, C. Constructive and destructive aspects of adaptive wormholes for the $5 \mathrm{~g}$ tactile internet. In Proceedings of the 9th ACM Conference on Security \& Privacy in Wireless and Mobile Networks, Darmstadt, Germany, 18-20 July 2016; pp. 109-120.

203. Santos, B.; Do, V.T.; Feng, B.; Do, T.V. Identity federation for cellular internet of things. In Proceedings of the 20187 th International Conference on Software and Computer Applications, Kuantan, Malaysia, 8-10 February 2018; pp. $223-228$. 
204. Hsieh, E.; Wang, H.; Liu, C.; Chung, S.S.; Chen, T.; Huang, S.; Chen, J.; Cheng, O. Embedded PUF on 14nm HKMG FinFET Platform: A Novel 2-bit-per-cell OTP-based Memory Feasible for IoT Secuirty Solution in 5G Era. In Proceedings of the 2019 Symposium on VLSI Technology, Kyoto, Japan, 9-14 June 2019; pp. T118-T119.

205. Abraham, S.; Weller, N. Next generation traffic event detection: A framework based on internet of vehicles (IoV). In Proceedings of the Second International Conference on Internet of things, Data and Cloud Computing, Cambridge, UK, 22-23 March 2017; pp. $1-3$.

206. Wang, N.; Jiao, L.; Wang, P.; Dabaghchian, M.; Zeng, K. Efficient identity spoofing attack detection for iot in mm-wave and massive mimo $5 \mathrm{~g}$ communication. In Proceedings of the 2018 IEEE Global Communications Conference (GLOBECOM), Abu Dhabi, United Arab Emirates, 9-13 December 2018; pp. 1-6.

207. Mao, B.; Kawamoto, Y.; Kato, N. AI-based joint optimization of QoS and security for 6G energy harvesting internet of things. IEEE Internet Things J. 2020, 7, 7032-7042. [CrossRef]

208. Stergiou, C.L.; Psannis, K.E.; Gupta, B.B. IoT-based Big Data secure management in the Fog over a 6G Wireless Network. IEEE Internet Things J. 2020. [CrossRef]

209. Porambage, P.; Gür, G.; Osorio, D.P.M.; Liyanage, M.; Gurtov, A.; Ylianttila, M. The Roadmap to 6G Security and Privacy. IEEE Open J. Commun. Soc. 2021. [CrossRef]

210. Gedeon, J.; Krisztinkovics, J.; Meurisch, C.; Stein, M.; Wang, L.; Mühlhäuser, M. A multi-cloudlet infrastructure for future smart cities: An empirical study. In Proceedings of the 1st International Workshop on Edge Systems, Analytics and Networking, Munich, Germany, 10-15 June 2018; pp. 19-24.

211. Mahdi, F.E.; Habbani, A.; Bouamoud, B.; Souidi, M. Bootstrapping services availability through multipath routing for enhanced security in urban iot. In Proceedings of the 4th International Conference on Smart City Applications, Casablanca, Morocco, 2-4 October 2019; pp. 1-9.

212. Musabe, R.; Ngoga, S.R.; Manizabayo, E.; Katambire, V.N.; Yaramba, H. Assessment and optimization of cooperative MIMO system for smart city application. In Proceedings of the 4th International Conference on Smart City Applications, Casablanca, Morocco, 2-4 October 2019; pp. 1-5.

213. Slalmi, A.; Saadane, R.; Chaibi, H.; Aroussi, H.K. Improving call admission control in 5G for smart cities applications. In Proceedings of the 4th International Conference on Smart City Applications, Casablanca, Morocco, 2-4 October 2019; pp. 1-6.

214. Benhaddou, D. Living building: A building block of smart cities. In Proceedings of the 2017 International Conference on Smart Digital Environment, Rabat, Morocco, 21-23 July 2017; pp. 182-188.

215. Rajaei, H.; Mirzaei, F. IoT, smart homes, and zigbee simulation. In Proceedings of the Communications and Networking Symposium, Baltimore, Maryland, 15-18 April 2018; pp. 1-10.

216. Mahjoubi, A.E.; Mazri, T.; Hmina, N. First Africa and Morocco NB-IoT experimental results and deployment scenario: New approach to improve main 5G KPIs for smart water management. In Proceedings of the Mediterranean Symposium on Smart City Application, Tangier, Morocco, 25-27 October 2017; pp. 1-6.

217. Raza, N.; Jabbar, S.; Han, J.; Han, K. Social vehicle-to-everything (V2X) communication model for intelligent transportation systems based on 5G scenario. In Proceedings of the 2nd International Conference on Future Networks and Distributed Systems, Amman, Jordan, 26-27 June 2018; pp. 1-8.

218. Wang, J.-X.; Liu, Y.; Lei, Z.-B.; Wu, K.-H.; Zhao, X.-Y.; Feng, C.; Liu, H.; Shuai, X.; Tang, Z.; Wu, L.; et al. Smart water lora IoT system. In Proceedings of the 2018 International Conference on Communication Engineering and Technology, Singapore, 24-26 February 2018; pp. 48-51.

219. Ding, A.Y.; Janssen, M. Opportunities for applications using 5G networks: Requirements, challenges, and outlook. In Proceedings of the Seventh International Conference on Telecommunications and Remote Sensing, Barcelona, Spain, 8-9 October 2018; pp. 27-34.

220. Lorimer, P.A.; Diec, V.M.-F.; Kantarci, B. Participatory detection of identity theft on mobile social platforms. In Proceedings of the 2017 IEEE Global Conference on Signal and Information Processing (GlobalSIP), Montreal, QC, Canada, 14-16 November 2017; pp. 833-837.

221. Dinh, D.V.; Yoon, B.-N.; Le, H.N.; Nguyen, U.Q.; Phan, K.D.; Pham, L.D. ICT enabling technologies for smart cities. In Proceedings of the 2020 22nd International Conference on Advanced Communication Technology (ICACT), Phoenix Park, Korea, 16-19 February 2020; pp. 1180-1192.

222. Xiao, K.; Zhao, J.; He, Y.; Yu, S. Trajectory prediction of UAV in smart city using recurrent neural networks. In Proceedings of the ICC 2019-2019 IEEE International Conference on Communications (ICC), Shanghai, China, 20-24 May 2019; pp. 1-6.

223. Allam, Z.; Jones, D.S. Future (post-COVID) digital, smart and sustainable cities in the wake of 6G: Digital twins, immersive realities and new urban economies. Land Use Policy 2021, 101, 105201. [CrossRef]

224. Woźniak, M.; Zielonka, A.; Sikora, A.; Piran, M.J.; Alamri, A. 6G-enabled IoT Home Environment control using Fuzzy Rules. IEEE Internet Things J. 2020. [CrossRef]

225. Janbi, N.; Katib, I.; Albeshri, A.; Mehmood, R. Distributed artificial intelligence-as-a-service (daiaas) for smarter ioe and $6 \mathrm{~g}$ environments. Sensors 2020, 20, 5796. [CrossRef] [PubMed]

226. Jamil, S.U.; Khan, M.A.; ur Rehman, S. Intelligent Task Off-Loading and Resource Allocation for 6G Smart City Environment. In Proceedings of the 2020 IEEE 45th Conference on Local Computer Networks (LCN), Sydney, NSW, Australia 16-19 November 2020; pp. 441-444. 
227. Oleśków-Szłapka, J.; Stachowiak, A.; Pawłowski, G.; Ellefsen, A.P.M.T. Multi-Agent Systems: A Case Study in an Onshore Oilfield That Explores Opportunities and Future Perspectives in Terms of lot, AI and 5G Technology. In Proceedings of the 2019 3rd International Conference on E-Business and Internet, Prague, Czech Republic, 9-11 November 2019; pp. 29-35.

228. Zhang, H.; Yang, Z.; Mohapatra, P. Wireless access to ultimate virtual reality 360-degree video. In Proceedings of the International Conference on Internet of Things Design and Implementation, Montreal, QC, Canada, 15-18 April 2019; pp. $271-272$.

229. Zhou, Y.; Liu, L.; Wang, L.; Hui, N.; Cui, X.; Wu, J.; Peng, Y.; Qi, Y.; Xing, C. Service aware 6G: An intelligent and open network based on convergence of communication, computing and caching. Digit. Commun. Netw. 2020, 6, 253-260. [CrossRef]

230. Katz, M.; Ahmed, I. Opportunities and challenges for visible light communications in 6G. In Proceedings of the 2020 2nd 6G wireless summit (6G SUMMIT), Levi, Finland, 17-20 March 2020; pp. 1-5.

231. Pawłowicz, B.; Salach, M.; Trybus, B. Smart city traffic monitoring system based on 5 G cellular network, RFID and machine learning. In Proceedings of the KKIO Software Engineering Conference, Pułtusk, Poland, 1 September 2018; pp. 151-165.

232. Lloret, J.; Parra, L.; Taha, M.; Tomás, J. An architecture and protocol for smart continuous eHealth monitoring using 5G. Comput. Netw. 2017, 129, 340-351. [CrossRef]

233. Lorimer, P.A.; Diec, V.M.-F.; Kantarci, B. COVERS-UP: Collaborative Verification of Smart User Profiles for social sustainability of smart cities. Sustain. Cities Soc. 2018, 38, 348-358. [CrossRef]

234. Han, Y.; Rao, B.D.; Lee, J. Massive uncoordinated access with massive MIMO: A dictionary learning approach. IEEE Trans. Wirel. Commun. 2019, 19, 1320-1332. [CrossRef]

235. Tian, X.; Huang, Y.; Verma, S.; Jin, M.; Ghosh, U.; Rabie, K.M.; ThuanDo, D. Power allocation scheme for maximizing spectral efficiency and energy efficiency tradeoff for uplink NOMA systems in B5G/6G. Phys. Commun. 2020, 43, 101227. [CrossRef]

236. Sodhro, A.H.; Pirbhulal, S.; Zongwei, L.; Muhammad, K.; Zahid, N. Towards 6G Architecture for Energy Efficient Communication in IoT-Enabled Smart Automation Systems. IEEE Internet Things J. 2020. [CrossRef]

237. Ramírez-Arroyo, A.; Zapata-Cano, P.H.; Palomares-Caballero, Á.; Carmona-Murillo, J.; Luna-Valero, F.; Valenzuela-Valdés, J.F. Multilayer Network Optimization for 5G \& 6G. IEEE Access 2020, 8, 204295-204308.

238. Verma, S.; Kaur, S.; Khan, M.A.; Sehdev, P.S. Towards Green Communication in 6G-enabled Massive Internet of Things. IEEE Internet Things J. 2020. [CrossRef]

239. Krundyshev, V.; Kalinin, M. Hybrid neural network framework for detection of cyber attacks at smart infrastructures. In Proceedings of the 12th International Conference on Security of Information and Networks, Sochi, Russia, 12-15 September 2019; pp. 1-7.

240. Fletcher, J.; Wallom, D. Using machine learning to orchestrate cloud resources in a RAN enabled edge environment. In Proceedings of the 17th Conference on Embedded Networked Sensor Systems, New York, NY, USA, 10-13 November 2019; pp. $452-453$.

241. Tseng, S.-C.; Liu, Z.-W.; Chou, Y.-C.; Huang, C.-W. Radio resource scheduling for 5G NR via deep deterministic policy gradient. In Proceedings of the 2019 IEEE International Conference on Communications Workshops (ICC Workshops), Shanghai, China, 20-24 May 2019; pp. 1-6.

242. Tayyaba, S.K.; Khattak, H.A.; Almogren, A.; Shah, M.A.; Din, I.U.; Alkhalifa, I.; Guizani, M. 5G vehicular network resource management for improving radio access through machine learning. IEEE Access 2020, 8, 6792-6800.

243. Ouyang, Y.; Li, Z.; Su, L.; Lu, W.; Lin, Z. APP-SON: Application characteristics-driven SON to optimize 4G/5G network performance and quality of experience. In Proceedings of the 2017 IEEE International Conference on Big Data (Big Data), Boston, MA, USA 11-14 December 2017; pp. 1514-1523.

244. Han, Z.; Liang, J. The analysis of node planning and control logic optimization of $5 \mathrm{G}$ wireless networks under deep mapping learning algorithms. IEEE Access 2019, 7, 156489-156499. [CrossRef]

245. Klautau, A.; Batista, P.; González-Prelcic, N.; Wang, Y.; Heath, R.W. 5G MIMO data for machine learning: Application to beam-selection using deep learning. In Proceedings of the 2018 Information Theory and Applications Workshop (ITA), San Diego, CA, USA, 11-16 February 2018; pp. 1-9.

246. Ho, C.-C.; Huang, B.-H.; Wu, M.-T.; Wu, T.-Y. Optimized Base Station Allocation for Platooning Vehicles Underway by Using Deep Learning Algorithm Based on 5G-V2X. In Proceedings of the 2019 IEEE 8th Global Conference on Consumer Electronics (GCCE), Osaka, Japan, 15-18 October 2019; pp. 1-2.

247. Joseph, S.; Misra, R.; Katti, S. Towards self-driving radios: Physical-layer control using deep reinforcement learning. In Proceedings of the 20th International Workshop on Mobile Computing Systems and Applications, Santa Cruz, CA, USA, 27-28 February 2019; pp. 69-74.

248. Liu, Q.; Han, T. When network slicing meets deep reinforcement learning. In Proceedings of the 15th International Conference on emerging Networking EXperiments and Technologies, Orlando, FL, USA, 9-12 December 2019. [CrossRef]

249. Villacrés, J.L.C.; Zhao, Z.; Braun, T.; Li, Z. A particle filter-based reinforcement learning approach for reliable wireless indoor positioning. IEEE J. Sel. Areas Commun. 2019, 37, 2457-2473. [CrossRef]

250. Zhang, X.; Liang, Y.-C.; Fang, J. Bayesian Inference Algorithms for Multiuser Detection in M2M Communications. In Proceedings of the 2016 IEEE 84th Vehicular Technology Conference (VTC-Fall), Montreal, QC, Canada, 18-21 September 2016; pp. 1-6.

251. Awan, D.A.; Cavalcante, R.L.; Yukawa, M.; Stanczak, S. Detection for 5G-NOMA: An online adaptive machine learning approach. In Proceedings of the 2018 IEEE International Conference on Communications (ICC), Kansas City, MO, USA, 20-24 May 2018; pp. 1-6. 
252. Asadi, A.; Müller, S.; Sim, G.H.; Klein, A.; Hollick, M. FML: Fast machine learning for 5 G mmWave vehicular communications. In Proceedings of the IEEE INFOCOM 2018-IEEE Conference on Computer Communications, Honolulu, HI, USA, 16-19 April 2018; pp. 1961-1969.

253. Guo, Y.; Wang, Z.; Li, M.; Liu, Q. Machine learning based mmWave channel tracking in vehicular scenario. In Proceedings of the 2019 IEEE International Conference on Communications Workshops (ICC Workshops), Shanghai, China, 20-24 May 2019; pp $1-6$.

254. Sullivan, K.; Tolan, M. Machine Learning for Terahertz Communication with Human-Implantable Devices. In Proceedings of the 2018 European Conference on Networks and Communications (EuCNC), Ljubljana, Slovenia, 18-21 June 2018; pp. $293-297$.

255. Guo, L.; Jin, B.; Yu, R.; Yao, C.; Sun, C.; Huang, D. Multi-label classification methods for green computing and application for mobile medical recommendations. IEEE Access 2016, 4, 3201-3209. [CrossRef]

256. Mohanta, B.; Das, P.; Patnaik, S. Healthcare 5.0: A paradigm shift in digital healthcare system using Artificial Intelligence, IOT and 5G Communication. In Proceedings of the 2019 International Conference on Applied Machine Learning (ICAML), Bhubaneswar, India, 25-26 May 2019; pp. 191-196.

257. Elkourdi, M.; Mazin, A.; Gitlin, R.D. Performance analysis for virtual-cell based CoMP 5 G networks using deep recurrent neural nets. In Proceedings of the 2019 Wireless Telecommunications Symposium (WTS), New York, NY, USA, 9-12 April 2019; pp. 1-6.

258. Motade, S.N.; Kulkarni, A.V. Channel estimation and data detection using machine learning for MIMO 5G communication systems in fading channel. Technologies 2018, 6, 72. [CrossRef]

259. Balevi, E.; Gitlin, R.D. Synergies between cloud-fag-thing and brain-spinal cord-nerve networks. In Proceedings of the 2018 Information theory and applications workshop (ITA), San Diego, CA, USA, 11-16 February 2018; pp. 1-9.

260. Balevi, E.; Gitlin, R.D. Unsupervised machine learning in 5G networks for low latency communications. In Proceedings of the 2017 IEEE 36th International Performance Computing and Communications Conference (IPCCC), San Diego, CA, USA, 10-12 December 2017; pp. 1-2.

261. Shen, Y.; Fang, W.; Ye, F.; Kadoch, M. EV charging behavior analysis using hybrid intelligence for 5G smart grid. Electronics 2020, 9, 80. [CrossRef]

262. Yan, S.; Jiao, M.; Zhou, Y.; Peng, M.; Daneshmand, M. Machine-Learning Approach for User Association and Content Placement in Fog Radio Access Networks. IEEE Internet Things J. 2020, 7, 9413-9425. [CrossRef]

263. Li, Y.; Chai, K.K.; Chen, Y.; Loo, J. Smart duty cycle control with reinforcement learning for machine to machine communications. In Proceedings of the 2015 IEEE International Conference on Communication Workshop (ICCW), London, UK, 8-12 June 2015; pp. 1458-1463.

264. Xu, F.; Hong, T.; Zhao, J.; Yang, T. Detection and identification technology of rotor unmanned aerial vehicles in 5G scene. Int. J. Distrib. Sens. Netw. 2019, 15, 1550147719853990. [CrossRef]

265. Healy, M.; Walsh, P. Detecting demeanor for healthcare with machine learning. In Proceedings of the 2017 IEEE International Conference on Bioinformatics and Biomedicine (BIBM), Kansas City, MO, USA, 13-16 November 2017; pp. $2015-2019$.

266. Sim, G.H.; Klos, S.; Asadi, A.; Klein, A.; Hollick, M. An online context-aware machine learning algorithm for $5 \mathrm{G}$ mmWave vehicular communications. IEEE/ACM Trans. Netw. 2018, 26, 2487-2500. [CrossRef]

267. Falkenberg, R.; Sliwa, B.; Piatkowski, N.; Wietfeld, C. Machine learning based uplink transmission power prediction for LTE and upcoming 5G networks using passive downlink indicators. In Proceedings of the 2018 IEEE 88th Vehicular Technology Conference (VTC-Fall), Chicago, IL, USA, 27-30 August 2018; pp. 1-7.

268. Yan, M.; Chen, B.; Feng, G.; Qin, S. Federated cooperation and augmentation for power allocation in decentralized wireless networks. IEEE Access 2020, 8, 48088-48100. [CrossRef]

269. Zhen, L.; Bashir, A.K.; Yu, K.; Al-Otaibi, Y.D.; Foh, C.H.; Xiao, P. Energy-efficient random access for LEO satellite-assisted 6G Internet of remote things. IEEE Internet Things J. 2020. [CrossRef]

270. Kouroshnezhad, S.; Peiravi, A.; Haghighi, M.S.; Jolfaei, A. Energy-Efficient Drone Trajectory Planning for the Localization of 6G-enabled IoT Devices. IEEE Internet Things J. 2020, 8, 5202-5210. [CrossRef]

271. Pokhrel, S.R. Federated learning meets blockchain at 6G edge: A drone-assisted networking for disaster response. In Proceedings of the 2nd ACM MobiCom Workshop on Drone Assisted Wireless Communications for 5G and Beyond, London, UK, 25 September 2020; pp. 49-54.

272. Deng, J.; Zeng, J.; Mai, S.; Jin, B.; Yuan, B.; You, Y.; Lu, S.; Yang, M. Analysis and prediction of ship energy efficiency using 6G big data internet of things and artificial intelligence technology. Int. J. Syst. Assur. Eng. Manag. 2021, 12, 824-834. [CrossRef]

273. Hadi, M.S.; Lawey, A.Q.; El-Gorashi, T.E.; Elmirghani, J.M. Patient-centric HetNets powered by machine learning and big data analytics for $6 \mathrm{G}$ networks. IEEE Access 2020, 8, 85639-85655. [CrossRef]

274. Liu, S.; Alfadhli, Y.M.; Shen, S.; Tian, H.; Chang, G.-K. Mitigation of multi-user access impairments in 5G A-RoF-based mobile fronthaul utilizing machine learning for an artificial neural network nonlinear equalizer. In Proceedings of the 2018 Optical Fiber Communications Conference and Exposition (OFC), San Diego, CA, USA, 11-15 March 2018; pp. 1-3.

275. Bega, D.; Gramaglia, M.; Banchs, A.; Sciancalepore, V.; Costa-Pérez, X. A machine learning approach to 5G infrastructure market optimization. IEEE Trans. Mob. Comput. 2019, 19, 498-512. [CrossRef]

276. Jia, Z.; Cheng, W.; Zhang, H. A partial learning-based detection scheme for massive MIMO. IEEE Wirel. Commun. Lett. 2019, 8 , 1137-1140. [CrossRef] 
277. Meng, Q.; Fang, X.; Yue, W.; Meng, Y.; Wei, J. Bayesian Network Prediction of Mobile User Throughput in 5G Wireless Networks. In Proceedings of the 2018 10th International Conference on Communications, Circuits and Systems (ICCCAS), Chengdu, China, 22-24 December 2018; pp. 291-295.

278. Yahia, I.G.B.; Bendriss, J.; Samba, A.; Dooze, P. CogNitive 5G networks: Comprehensive operator use cases with machine learning for management operations. In Proceedings of the 2017 20th Conference on Innovations in Clouds, Internet and Networks (ICIN), Paris, France, 7-9 March 2017; pp. 252-259.

279. Luo, H.; Xu, J.; Zhang, J.; Zhang, P.; Huang, L. Deep Learning Based Antenna Selection Aided Space-Time Shift Keying Systems. In Proceedings of the Proceedings of the 2019 International Conference on Artificial Intelligence and Computer Science, Wuhan, China, 12-13 July 2019; pp. 409-413.

280. Maimó, L.F.; Clemente, F.J.G.; Pérez, M.G.; Pérez, G.M. On the performance of a deep learning-based anomaly detection system for 5G networks. In Proceedings of the 2017 IEEE SmartWorld, Ubiquitous Intelligence \& Computing, Advanced \& Trusted Computed, Scalable Computing \& Communications, Cloud \& Big Data Computing, Internet of People and Smart City Innovation (SmartWorld/SCALCOM/UIC/ATC/CBDCom/IOP/SCI), San Francisco, CA, USA, 4-8 August 2017; pp. 1-8.

281. Khunteta, S.; Chavva, A.K.R. Deep learning based link failure mitigation. In Proceedings of the 2017 16th IEEE International Conference on Machine Learning and Applications (ICMLA), Cancun, Mexico, 18-21 December 2017; pp. 806-811.

282. Borgerding, M.; Schniter, P.; Rangan, S. AMP-inspired deep networks for sparse linear inverse problems. IEEE Trans. Signal Process. 2017, 65, 4293-4308. [CrossRef]

283. Kim, K.; Lee, J.; Choi, J. Deep learning based pilot allocation scheme (DL-PAS) for 5G massive MIMO system. IEEE Commun. Lett. 2018, 22, 828-831. [CrossRef]

284. Bega, D.; Gramaglia, M.; Fiore, M.; Banchs, A.; Costa-Perez, X. DeepCog: Cognitive network management in sliced 5G networks with deep learning. In Proceedings of the IEEE INFOCOM 2019-IEEE Conference on Computer Communications, Paris, France, 29 April-2 May 2019; pp. 280-288.

285. Thantharate, A.; Paropkari, R.; Walunj, V.; Beard, C. Deepslice: A deep learning approach towards an efficient and reliable network slicing in 5G networks. In Proceedings of the 2019 IEEE 10th Annual Ubiquitous Computing, Electronics \& Mobile Communication Conference (UEMCON), New York, NY, USA, 10-12 October 2019; pp. 762-767.

286. Hughes, B.; Bothe, S.; Farooq, H.; Imran, A. Generative adversarial learning for machine learning empowered self organizing 5G networks. In Proceedings of the 2019 International Conference on Computing, Networking and Communications (ICNC), Honolulu, HI, USA, 18-21 February 2019; pp. 282-286.

287. Yan, M.; Feng, G.; Zhou, J.; Sun, Y.; Liang, Y.-C. Intelligent resource scheduling for $5 \mathrm{G}$ radio access network slicing. IEEE Trans. Veh. Technol. 2019, 68, 7691-7703. [CrossRef]

288. Hao, H.; Xu, C.; Wang, M.; Xie, H.; Liu, Y.; Wu, D.O. Knowledge-centric proactive edge caching over mobile content distribution network. In Proceedings of the IEEE INFOCOM 2018-IEEE Conference on Computer Communications Workshops (INFOCOM WKSHPS), Honolulu, HI, USA, 15-19 April 2018; pp. 450-455.

289. Cao, J.; Peng, T.; Dong, W.; Liu, X.; Wang, W. An association rules based conflict-graph construction approach for ultra-dense networks. In Proceedings of the 2018 IEEE Globecom Workshops (GC Wkshps), Abu Dhabi, United Arab Emirates, 9-13 December 2018; pp. 1-7.

290. Schwarzmann, S.; Marquezan, C.C.; Bosk, M.; Liu, H.; Trivisonno, R.; Zinner, T. Estimating video streaming QoE in the 5G architecture using machine learning. In Proceedings of the Proceedings of the 4th Internet-QoE Workshop on QoE-based Analysis and Management of Data Communication Networks, Los Cabos, Mexico, 21 October 2019; pp. 7-12.

291. Kao, W.-C.; Zhan, S.-Q.; Lee, T.-S. AI-Aided 3-D Beamforming for Millimeter Wave Communications. In Proceedings of the 2018 International Symposium on Intelligent Signal Processing and Communication Systems (ISPACS), Ishigaki, Japan, 27-30 November 2018; pp. 278-283.

292. Imtiaz, S.; Ghauch, H.; Rahman, M.M.U.; Koudouridis, G.; Gross, J. Learning-based resource allocation scheme for TDD-based 5G CRAN system. In Proceedings of the 19th ACM International Conference on Modeling, Analysis and Simulation of Wireless and Mobile Systems, Malta, Malta, 13-17 November 2016; pp. 176-185.

293. VKafle, P.; Fukushima, Y.; Martinez-Julia, P.; Miyazawa, T. Consideration on automation of 5 G network slicing with machine learning. In Proceedings of the 2018 ITU Kaleidoscope: Machine Learning for a 5G Future (ITU K), Santa Fe, Argentina, 26-28 November 2018; pp. 1-8.

294. Ma, B.; Yang, B.; Zhu, Y.; Zhang, J. Context-aware proactive 5G load balancing and optimization for urban areas. IEEE Access 2020, 8, 8405-8417. [CrossRef]

295. Jiang, W.; Strufe, M.; Schotten, H.D. A SON decision-making framework for intelligent management in 5G mobile networks. In Proceedings of the 2017 3rd IEEE International Conference on Computer and Communications (ICCC), Chengdu, China, 13-16 December 2017; pp. 1158-1162.

296. Jiang, W.; Strufe, M.; Gundall, M.; Schotten, H.D. An SDN/NFV Proof-of-Concept Test-Bed for Machine Learning-Based Network Management. In Proceedings of the 2018 IEEE 4th International Conference on Computer and Communications (ICCC), Chengdu, China, 7-10 December 2018; pp. 1966-1971.

297. Careglio, D.; Spadaro, S.; Cabellos, A.; Lazaro, J.; Perelló, J.; Barlet, P.; Gené, J.M.; Paillissé, J. ALLIANCE Project: Architecting a knowledge-defined 5G-enabled network infrastructure. In Proceedings of the 2018 20th International Conference on Transparent Optical Networks (ICTON), Bucharest, Romania, 1-5 July 2018; pp. 1-6. 
298. Balapuwaduge, I.A.; Li, F.Y. Hidden Markov Model Based Machine Learning for mMTC Device Cell Association in 5G Networks. In Proceedings of the ICC 2019-2019 IEEE International Conference on Communications (ICC), Shanghai, China, 20-24 May 2019; pp. 1-6.

299. Bosneag, A.-M.; Wang, M.X. Intelligent network management mechanisms as a step towards SG. In Proceedings of the 20178 th International Conference on the Network of the Future (NOF), London, UK, 22-24 November 2017; pp. 52-57.

300. Ahmed, I.; Khammari, H. Joint machine learning based resource allocation and hybrid beamforming design for massive MIMO systems. In Proceedings of the 2018 IEEE Globecom Workshops (GC Wkshps), Abu Dhabi, United Arab Emirates, 9-13 December 2018; pp. 1-6.

301. Yazar, A.; Arslan, H. Selection of waveform parameters using machine learning for 5G and beyond. In Proceedings of the 2019 IEEE 30th Annual International Symposium on Personal, Indoor and Mobile Radio Communications (PIMRC), Istanbul, Turkey, 8-11 September 2019; pp. 1-6.

302. Strodthoff, N.; Göktepe, B.; Schierl, T.; Samek, W.; Hellge, C. Machine learning for early HARQ feedback prediction in 5G. In Proceedings of the 2018 IEEE Globecom Workshops (GC Wkshps), Abu Dhabi, United Arab Emirates, 9-13 December 2018; pp. $1-6$.

303. Perez, J.S.; Jayaweera, S.K.; Lane, S. Machine learning aided cognitive RAT selection for 5G heterogeneous networks. In Proceedings of the 2017 IEEE International Black Sea Conference on Communications and Networking (BlackSeaCom), Istanbul, Turkey, 5-8 June 2017; pp. 1-5.

304. Li, J.; Zhao, Z.; Li, R. Machine learning-based IDS for software-defined 5G network. IET Netw. 2017, 7, 53-60. [CrossRef]

305. Khan, T.A.; Mehmood, A.; Rivera, J.J.D.; Song, W.-C. Machine learning approach for automatic configuration and management of $5 \mathrm{~g}$ platforms. In Proceedings of the 2019 20th Asia-Pacific Network Operations and Management Symposium (APNOMS), Matsue, Japan, 18-20 September 2019; pp. 1-6.

306. Salhab, N.; Rahim, R.; Langar, R.; Boutaba, R. Machine learning based resource orchestration for $5 \mathrm{~g}$ network slices. In Proceedings of the 2019 IEEE Global Communications Conference (GLOBECOM), Waikoloa, HI, USA, 9-13 December 2019; pp. 1-6.

307. Gupta, R.K.; Misra, R. Machine Learning-based Slice allocation Algorithms in 5G Networks. In Proceedings of the 2019 International Conference on Advances in Computing, Communication and Control (ICAC3), Mumbai, India, 20-21 December 2019; pp. 1-4.

308. Ahmadi, H.; Fontanesi, G.; Katzis, K.; Shakir, M.Z.; Zhu, A. Resilience of airborne networks. In Proceedings of the 2018 IEEE 29th Annual International Symposium on Personal, Indoor and Mobile Radio Communications (PIMRC), Bologna, Italy, 9-12 September 2018; pp. 1-2.

309. Ali-Tolppa, J.; Kocsis, S.; Schultz, B.; Bodrog, L.; Kajo, M. Self-healing and resilience in future 5G cognitive autonomous networks. In Proceedings of the 2018 ITU Kaleidoscope: Machine Learning for a 5G Future (ITU K), Santa Fe, Argentina, 26-28 November 2018; pp. 1-8.

310. Li, Y.; Piao, X.; Zhang, H.; Lei, K. NDN producer mobility management based on echo state network: A lightweight machine learning approach. In Proceedings of the 2018 IEEE 24th International Conference on Parallel and Distributed Systems (ICPADS), Singapore, 11-13 December 2018; pp. 275-282.

311. Mwanje, S.S.; Mannweiler, C. Towards cognitive autonomous networks in 5g. In Proceedings of the 2018 ITU Kaleidoscope: Machine Learning for a 5G Future (ITU K), Santa Fe, Argentina, 26-28 November 2018; pp. 1-8.

312. Bashir, A.K.; Arul, R.; Basheer, S.; Raja, G.; Jayaraman, R.; Qureshi, N.M.F. An optimal multitier resource allocation of cloud RAN in $5 \mathrm{G}$ using machine learning. Trans. Emerg. Telecommun. Technol. 2019, 30, e3627. [CrossRef]

313. Murudkar, C.V.; Gitlin, R.D. User-centric approaches for next-generation self-organizing wireless communication networks using machine learning. In Proceedings of the 2019 IEEE International Conference on Microwaves, Antennas, Communications and Electronic Systems (COMCAS), Tel-Aviv, Israel, 4-6 November 2019; pp. 1-6.

314. Awan, D.A.; Cavalcante, R.L.; Stanczak, S. Robust Cell-Load Learning With a Small Sample Set. IEEE Trans. Signal Process. 2019, 68, 270-283. [CrossRef]

315. Mozaffari, M.; Kasgari, A.T.Z.; Saad, W.; Bennis, M.; Debbah, M. Beyond 5G with UAVs: Foundations of a 3D wireless cellular network. IEEE Trans. Wirel. Commun. 2018, 18, 357-372. [CrossRef]

316. Zhang, J.; Xu, X.; Zhang, K.; Zhang, B.; Tao, X.; Zhang, P. Machine learning based flexible transmission time interval scheduling for eMBB and uRLLC coexistence scenario. IEEE Access 2019, 7, 65811-65820. [CrossRef]

317. Ibarrola, E.; Davis, M.; Voisin, C.; Close, C.; Cristobo, L. A machine learning management model for qoe enhancement in next-generation wireless ecosystems. In Proceedings of the 2018 ITU Kaleidoscope: Machine Learning for a 5G Future (ITU K), Santa Fe, Argentina, 26-28 November 2018; pp. 1-8.

318. Zhu, G.; Zan, J.; Yang, Y.; Qi, X. A supervised learning based QoS assurance architecture for 5G networks. IEEE Access 2019, 7, 43598-43606. [CrossRef]

319. Qin, M.; Yang, Q.; Cheng, N.; Zhou, H.; Rao, R.R.; Shen, X. Machine learning aided context-aware self-healing management for ultra dense networks with QoS provisions. IEEE Trans. Veh. Technol. 2018, 67, 12339-12351. [CrossRef]

320. Martin, A.; Egaña, J.; Flórez, J.; Montalbán, J.; Olaizola, I.G.; Quartulli, M.; Viola, R.; Zorrilla, M. Network resource allocation system for QoE-aware delivery of media services in 5G networks. IEEE Trans. Broadcast. 2018, 64, 561-574. [CrossRef]

321. Ilievski, G.; Latkoski, P. Efficiency of Supervised Machine Learning Algorithms in Regular and Encrypted VoIP Classification within NFV Environment. Radioengineering 2020, 29, 243-250. [CrossRef] 
322. Sciancalepore, V.; Costa-Perez, X.; Banchs, A. RL-NSB: Reinforcement learning-based 5G network slice broker. IEEE/ACM Trans. Netw. 2019, 27, 1543-1557. [CrossRef]

323. Vita, F.D.; Bruneo, D.; Puliafito, A.; Nardini, G.; Virdis, A.; Stea, G. A deep reinforcement learning approach for data migration in multi-access edge computing. In Proceedings of the 2018 ITU Kaleidoscope: Machine Learning for a 5G Future (ITU K), Santa Fe, Argentina, 26-28 November 2018; pp. 1-8.

324. Shahriari, B.; Moh, M.; Moh, T.-S. Generic Online Learning for Partial Visible Dynamic Environment with Delayed Feedback: Online Learning for 5G C-RAN Load-Balancer. In Proceedings of the 2017 International Conference on High Performance Computing \& Simulation (HPCS), Genoa, Italy, 17-21 July 2017; pp. 176-185.

325. Wang, X.; Li, J.; Wang, L.; Yang, C.; Han, Z. Intelligent user-centric network selection: A model-driven reinforcement learning framework. IEEE Access 2019, 7, 21645-21661. [CrossRef]

326. Zineb, A.B.; Ayadi, M.; Tabbane, S. A novel cognitive architecture for QoS/QoE management in NextG Networks based on Q-learning and R-MLP approaches. In Proceedings of the 2016 International Wireless Communications and Mobile Computing Conference (IWCMC), Paphos, Cyprus, 5-9 September 2016; pp. 892-897.

327. Murudkar, C.V.; Gitlin, R.D. Optimal-capacity, shortest path routing in self-organizing $5 \mathrm{G}$ networks using machine learning. In Proceedings of the 2019 IEEE 20th Wireless and Microwave Technology Conference (WAMICON), Cocoa Beach, FL, USA, 8-9 April 2019; pp. 1-5.

328. Puspita, R.H.; Shah, S.D.A.; Lee, G.-m.; Roh, B.-h.; Oh, J.; Kang, S. Reinforcement learning based 5 G enabled cognitive radio networks. In Proceedings of the 2019 International Conference on Information and Communication Technology Convergence (ICTC), Jeju, Korea, 16-18 October 2019; pp. 555-558.

329. Troia, S.; Alvizu, R.; Maier, G. Reinforcement learning for service function chain reconfiguration in NFV-SDN metro-core optical networks. IEEE Access 2019, 7, 167944-167957. [CrossRef]

330. Bikov, E.; Botvich, D. Smart concurrent learning scheme for $5 \mathrm{G}$ network: QoS-aware radio resource allocation. In Proceedings of the 2017 IVth International Conference on Engineering and Telecommunication (EnT), Moscow, Russia, 29-30 November 2017; pp. 99-103.

331. Abiko, Y.; Saito, T.; Ikeda, D.; Ohta, K.; Mizuno, T.; Mineno, H. Flexible resource block allocation to multiple slices for radio access network slicing using deep reinforcement learning. IEEE Access 2020, 8, 68183-68198. [CrossRef]

332. Sreedevi, A.; Rao, T.R. Reinforcement learning algorithm for $5 \mathrm{G}$ indoor device-to-device communications. Trans. Emerg. Telecommun. Technol. 2019, 30, e3670 [CrossRef]

333. Imtiaz, S.; Koudouridis, G.P.; Ghauch, H.; Gross, J. Random forests for resource allocation in 5 G cloud radio access networks based on position information. EURASIP J. Wirel. Commun. Netw. 2018, 2018. [CrossRef]

334. Imtiaz, S.; Ghauch, H.; Koudouridis, G.P.; Gross, J. Random forests resource allocation for 5G systems: Performance and robustness study. In Proceedings of the 2018 IEEE Wireless Communications and Networking Conference Workshops (WCNCW), Barcelona, Spain, 15-18 April 2018; pp. 326-331.

335. Daga, H.; Nicholson, P.K.; Gavrilovska, A.; Lugones, D. Cartel: A system for collaborative transfer learning at the edge. In Proceedings of the ACM Symposium on Cloud Computing, Santa Cruz, CA, USA, 20-23 November 2019; pp. 25-37.

336. Chergui, H.; Tourki, K.; Lguensat, R.; Benjillali, M.; Verikoukis, C.; Debbah, M. Classification algorithms for semi-blind uplink/downlink decoupling in sub-6 GHz/mmwave 5G networks. In Proceedings of the 2019 15th International Wireless Communications \& Mobile Computing Conference (IWCMC), Tangier, Morocco, 24-28 June 2019; pp. 2031-2035.

337. Yang, Y.; Deng, X.; He, D.; You, Y.; Song, R. Machine learning inspired codeword selection for dual connectivity in 5G user-centric ultra-dense networks. IEEE Trans. Veh. Technol. 2019, 68, 8284-8288. [CrossRef]

338. Dai, L.; Zhang, H.; Zhuang, Y. Propagation-model-free coverage evaluation via machine learning for future $5 \mathrm{G}$ networks. In Proceedings of the 2018 IEEE 29th Annual International Symposium on Personal, Indoor and Mobile Radio Communications (PIMRC), Bologna, Italy, 9-12 September 2018; pp. 1-5.

339. Gebrie, H.; Farooq, H.; Imran, A. What machine learning predictor performs best for mobility prediction in cellular networks? In Proceedings of the 2019 IEEE International Conference on Communications Workshops (ICC Workshops), Shanghai, China, 20-24 May 2019; pp. 1-6.

340. Xie, Y.; Ji, W.; Li, T.; Liang, Y.; Li, F. Location Aided and Machine Learning-Based Beam Allocation for 3D Massive MIMO Systems. In Proceedings of the 2019 15th International Wireless Communications \& Mobile Computing Conference (IWCMC), Tangier, Morocco, 24-28 June 2019; pp. 836-841.

341. Sun, D.; Willmann, S. Deep learning-based dependability assessment method for industrial wireless network. IFAC-PapersOnLine 2019, 52, 219-224. [CrossRef]

342. Kasongo, S.M.; Sun, Y. A deep learning method with wrapper based feature extraction for wireless intrusion detection system. Comput. Secur. 2020, 92, 101752. [CrossRef]

343. Lin, J.; Feng, S.; Zhang, Y.; Yang, Z.; Zhang, Y. A novel deep neural network based approach for sparse code multiple access. Neurocomputing 2020, 382, 52-63. [CrossRef]

344. Asghar, M.Z.; Abbas, M.; Zeeshan, K.; Kotilainen, P.; Hämäläinen, T. Assessment of deep learning methodology for self-organizing 5g networks. Appl. Sci. 2019, 9, 2975. [CrossRef]

345. Zorzi, M.; Zanella, A.; Testolin, A.; Grazia, M.D.F.D.; Zorzi, M. Cognition-based networks: A new perspective on network optimization using learning and distributed intelligence. IEEE Access 2015, 3, 1512-1530. [CrossRef] 
346. Mo, J.; Ng, B.L.; Chang, S.; Huang, P.; Kulkarni, M.N.; AlAmmouri, A.; Zhang, J.; Lee, J.; Choi, W.-J. Beam codebook design for 5G mmWave terminals. IEEE Access 2019, 7, 98387-98404. [CrossRef]

347. Wang, H.; Wu, Y.; Min, G.; Xu, J.; Tang, P. Data-driven dynamic resource scheduling for network slicing: A deep reinforcement learning approach. Inf. Sci. 2019, 498, 106-116. [CrossRef]

348. Strodthoff, N.; Göktepe, B.; Schierl, T.; Hellge, C.; Samek, W. Enhanced machine learning techniques for early HARQ feedback prediction in 5G. IEEE J. Sel. Areas Commun. 2019, 37, 2573-2587. [CrossRef]

349. Huang, J.; Wang, C.-X.; Bai, L.; Sun, J.; Yang, Y.; Li, J.; Tirkkonen, O.; Zhou, M. A big data enabled channel model for $5 \mathrm{G}$ wireless communication systems. IEEE Trans. Big Data 2018, 6, 211-222. [CrossRef]

350. Ha, C.-B.; You, Y.-H.; Song, H.-K. Machine learning model for adaptive modulation of multi-stream in MIMO-OFDM system. IEEE Access 2018, 7, 5141-5152. [CrossRef]

351. Thrane, J.; Zibar, D.; Christiansen, H.L. Model-aided deep learning method for path loss prediction in mobile communication systems at 2.6 GHz. IEEE Access 2020, 8, 7925-7936. [CrossRef]

352. Cho, Y.-J.; Kim, Y.-S.; Kim, S.; Sim, D.; Kwak, D.; Lee, J. AI-Enabled Wireless KPI Monitoring and DiagnosisSystem for 5G Cellular Networks. In Proceedings of the 2019 International Conference on Information and Communication Technology Convergence (ICTC), Jeju, Korea, 16-18 October 2019; pp. 899-901.

353. Subramanya, T.; Harutyunyan, D.; Riggio, R. Machine learning-driven service function chain placement and scaling in MECenabled 5G networks. Comput. Netw. 2020, 166, 106980. [CrossRef]

354. AbdelMoniem, M.; Gasser, S.M.; El-Mahallawy, M.S.; Fakhr, M.W.; Soliman, A. Enhanced NOMA system using adaptive coding and modulation based on LSTM neural network channel estimation. Appl. Sci. 2019, 9, 3022. [CrossRef]

355. Cao, J.; Liu, X.; Dong, W.; Peng, T.; Duan, R.; Yuan, Y.; Wang, W. A neural network based conflict-graph construction approach for ultra-dense networks. In Proceedings of the 2018 IEEE Globecom Workshops (GC Wkshps), Abu Dhabi, United Arab Emirates, 9-13 December 2018; pp. 1-6.

356. Huang, C.-W.; Chiang, C.-T.; Li, Q. A study of deep learning networks on mobile traffic forecasting. In Proceedings of the 2017 IEEE 28th Annual International Symposium on Personal, Indoor, and Mobile Radio Communications (PIMRC), Montreal, QC, Canada, 8-13 October 2017; pp. 1-6.

357. Hussain, B.; Du, Q.; Ren, P. Deep learning-based big data-assisted anomaly detection in cellular networks. In Proceedings of the 2018 IEEE Global Communications Conference (GLOBECOM), Abu Dhabi, United Arab Emirates, 9-13 December 2018; pp. 1-6.

358. Le, L.-V.; Sinh, D.; Tung, L.-P.; Lin, B.-S.P. A practical model for traffic forecasting based on big data, machine-learning, and network KPIs. In Proceedings of the 2018 15th IEEE Annual Consumer Communications \& Networking Conference (CCNC), Las Vegas, NV, USA, 12-15 January 2018; pp. 1-4.

359. Nurminen, J.K.; Mfula, H. A unified framework for 5G network management tools. In Proceedings of the 2018 IEEE 11th Conference on Service-Oriented Computing and Applications (SOCA), Paris, France, 20-22 November 2018; pp. 41-48.

360. Chiu, P.; Reunanen, J.; Luostari, R.; Holma, H. Big Data Analytics for 4.9 G and 5G Mobile Network Optimization. In Proceedings of the 2017 IEEE 85th Vehicular Technology Conference (VTC Spring), Sydney, NSW, Australia, 4-7 June 2017; pp. 1-4.

361. Tung, L.-P.; Lin, B.-S.P. Big data and machine learning driven handover management and forecasting. In Proceedings of the 2017 IEEE Conference on Standards for Communications and Networking (CSCN), Helsinki, Finland, 4-7 June 2017; pp. $214-219$.

362. Baştuğ, E.; Bennis, M.; Zeydan, E.; Kader, M.A.; Karatepe, I.A.; Er, A.S.; Debbah, M. Big data meets telcos: A proactive caching perspective. J. Commun. Netw. 2015, 17, 549-557. [CrossRef]

363. Keshavamurthy, B.; Ashraf, M. Conceptual design of proactive SONs based on the big data framework for 5G cellular networks: A novel machine learning perspective facilitating a shift in the son paradigm. In Proceedings of the 2016 International Conference System Modeling \& Advancement in Research Trends (SMART), Moradabad, India, 25-27 November 2016; pp. 298-304.

364. Kader, M.A.; Bastug, E.; Bennis, M.; Zeydan, E.; Karatepe, A.; Er, A.S.; Debbah, M. Leveraging big data analytics for cache-enabled wireless networks. In Proceedings of the 2015 IEEE Globecom Workshops (GC Wkshps), San Diego, CA, USA, 6-10 December 2015; pp. 1-6.

365. López-Pires, F.; Barán, B. Machine learning opportunities in cloud computing data center management for 5G services. In Proceedings of the 2018 ITU Kaleidoscope: Machine Learning for a 5G Future (ITU K), Santa Fe, Argentina, 26-28 November 2018; pp. 1-6.

366. Le, L.-V.; Lin, B.-S.P.; Tung, L.-P.; Sinh, D. SDN/NFV, machine learning, and big data driven network slicing for 5G. In Proceedings of the 2018 IEEE 5G World Forum (5GWF), Silicon Valley, CA, USA, 9-11 July 2018; pp. 20-25.

367. Le, L.-V.; Sinh, D.; Lin, B.-S.P.; Tung, L.-P. Applying big data, machine learning, and SDN/NFV to 5 G traffic clustering, forecasting, and management. In Proceedings of the 2018 th IEEE Conference on Network Softwarization and Workshops (NetSoft), Montreal, QC, Canada, 25-29 June 2018; pp. 168-176.

368. Paul, U.; Liu, J.; Troia, S.; Falowo, O.; Maier, G. Traffic-profile and machine learning based regional data center design and operation for 5G network. J. Commun. Netw. 2019, 21, 569-583. [CrossRef]

369. Maimó, L.F.; Gómez, Á.L.P.; Clemente, F.J.G.; Pérez, M.G.; Pérez, G.M. A self-adaptive deep learning-based system for anomaly detection in $5 \mathrm{G}$ networks. IEEE Access 2018, 6, 7700-7712. [CrossRef]

370. Zhao, G.; Yang, H.; Yu, A.; Zhu, Y.; Li, K.; Zhang, J. Leveraging Deep Learning to Predict Cyberattack with Traffic Whitelist for Optical Fronthaul Networks in 5G and Beyond. In Proceedings of the Asia Communications and Photonics Conference, Chengdu, China, 2-5 November 2019; p. S3C.5. 
371. Munisankaraiah, S.; Kumar, A.A. Physical layer security in 5G wireless networks for data protection. In Proceedings of the 2016 2nd International Conference on Next Generation Computing Technologies (NGCT), Dehradun, India, 14-16 October 2016; pp. 883-887.

372. Covaci, S.; Repetto, M.; Risso, F. Towards Autonomous Security Assurance in 5G Infrastructures. IEICE Trans. Commun. 2018. [CrossRef]

373. Ravi, N.; Rani, P.V.; Shalinie, S.M. Secure Deep Neural (SeDeN) Framework for 5G Wireless Networks. In Proceedings of the 2019 10th International Conference on Computing, Communication and Networking Technologies (ICCCNT), Kanpur, India, 6-8 July 2019; pp. 1-6.

374. Sim, M.S.; Lim, Y.-G.; Park, S.H.; Dai, L.; Chae, C.-B. Deep learning-based mmWave beam selection for 5G NR/6G with sub-6 $\mathrm{GHz}$ channel information: Algorithms and prototype validation. IEEE Access 2020, 8, 51634-51646. [CrossRef]

375. Lauridsen, M.; Mogensen, P.; Sorensen, T.B. Estimation of a $10 \mathrm{gb} / \mathrm{s} 5 \mathrm{G}$ receiver's performance and power evolution towards 2030. In Proceedings of the 2015 IEEE 82nd Vehicular Technology Conference (VTC2015-Fall), Boston, MA, USA, 6-9 September 2015; pp. 1-5.

376. Zong, B.; Fan, C.; Wang, X.; Duan, X.; Wang, B.; Wang, J. 6G technologies: Key drivers, core requirements, system architectures, and enabling technologies. IEEE Veh. Technol. Mag. 2019, 14, 18-27. [CrossRef]

377. Elmeadawy, S.; Shubair, R.M. Enabling technologies for 6G future wireless communications: Opportunities and challenges. arXiv 2020, arXiv:2002.06068.

378. Lu, Y.; Zheng, X. 6G: A survey on technologies, scenarios, challenges, and the related issues. J. Ind. Inf. Integr. 2020, 19, 100158. [CrossRef]

379. Xu, X.; Li, D.; Dai, Z.; Li, S.; Chen, X. A heuristic offloading method for deep learning edge services in 5G networks. IEEE Access 2019, 7, 67734-67744. [CrossRef]

380. Qian, B.; Zhou, H.; Ma, T.; Yu, K.; Yu, Q.; Shen, X. Multi-Operator Spectrum Sharing for Massive IoT Coexisting in 5G/B5G Wireless Networks. IEEE J. Sel. Areas Commun. 2020. [CrossRef]

381. Liu, B.; Liu, C.; Peng, M. Resource allocation for energy-efficient MEC in NOMA-enabled massive IoT networks. IEEE J. Sel. Areas Commun. 2020. [CrossRef]

382. Jiang, N.; Deng, Y.; Nallanathan, A.; Kang, X.; Quek, T.Q. Analyzing random access collisions in massive IoT networks. IEEE Trans. Wirel. Commun. 2018, 17, 6853-6870. [CrossRef]

383. Kim, T.; Jung, B.C. Performance analysis of grant-free multiple access for supporting sporadic traffic in massive IoT networks. IEEE Access 2019, 7, 166648-166656. [CrossRef]

384. Qi, Q.; Chen, X.; Ng, D.W.K. Robust beamforming for NOMA-based cellular massive IoT with SWIPT. IEEE Trans. Signal Process. 2019, 68, 211-224. [CrossRef]

385. Alsharif, M.H.; Kim, J.; Kim, J.H. Green and sustainable cellular base stations: An overview and future research directions. Energies 2017, 10, 587. [CrossRef]

386. Memon, M.L.; Saxena, N.; Roy, A.; Shin, D.R. Backscatter communications: Inception of the battery-free era-A comprehensive survey. Electronics 2019, 8, 129. [CrossRef]

387. Huang, C.; Hu, S.; Alexandropoulos, G.C.; Zappone, A.; Yuen, C.; Zhang, R.; Renzo, M.D.; Debbah, M. Holographic MIMO surfaces for 6G wireless networks: Opportunities, challenges, and trends. IEEE Wirel. Commun. 2020, 27, 118-125. [CrossRef]

388. Liu, Q.; Wu, J.; Xia, P.; Zhao, S.; Chen, W.; Yang, Y.; Hanzo, L. Charging unplugged: Will distributed laser charging for mobile wireless power transfer work? IEEE Veh. Technol. Mag. 2016, 11, 36-45. [CrossRef]

389. Huynh, N.V.; Hoang, D.T.; Lu, X.; Niyato, D.; Wang, P.; Kim, D.I. Ambient backscatter communications: A contemporary survey. IEEE Commun. Surv. Tutor. 2018, 20, 2889-2922. [CrossRef]

390. Gentry, C. Computing arbitrary functions of encrypted data. Commun. ACM 2010, 53, 97-105. [CrossRef]

391. Ferraro, P.; King, C.; Shorten, R. Distributed ledger technology for smart cities, the sharing economy, and social compliance. IEEE Access 2018, 6, 62728-62746. [CrossRef]

392. Silver, D.; Schrittwieser, J.; Simonyan, K.; Antonoglou, I.; Huang, A.; Guez, A.; Hubert, T.; Baker, L.; Lai, M.; Bolton, A.; et al.Mastering the game of go without human knowledge. Nature 2017, 550, 354-359. [CrossRef] [PubMed]

393. Letaief, K.B.; Chen, W.; Shi, Y.; Zhang, J.; Zhang, Y.-J.A. The roadmap to 6G: AI empowered wireless networks. IEEE Commun. Mag. 2019, 57, 84-90. [CrossRef]

394. David, K.; Berndt, H. 6G vision and requirements: Is there any need for beyond 5G? IEEE Veh. Technol. Mag. 2018, 13, 72-80. [CrossRef]

395. Li, R. Towards a new internet for the year 2030 and beyond. In Proceedings of the 3rd Annu. ITU IMT-2020/5G Workshop Demo Day, Geneva, Switzerland, 18 July 2018; pp. 1-21.

396. Berardinelli, G.; Mahmood, N.H.; Rodriguez, I.; Mogensen, P. Beyond 5G wireless IRT for industry 4.0: Design principles and spectrum aspects. In Proceedings of the 2018 IEEE Globecom Workshops (GC Wkshps), Abu Dhabi, United Arab Emirates, 9-13 December 2018; pp. 1-6.

397. Botsinis, P.; Alanis, D.; Babar, Z.; Nguyen, H.V.; Chandra, D.; Ng, S.X.; Hanzo, L. Quantum search algorithms for wireless communications. IEEE Commun. Surv. Tutor. 2018, 21, 1209-1242. [CrossRef]

398. Akan, O.B.; Ramezani, H.; Khan, T.; Abbasi, N.A.; Kuscu, M. Fundamentals of molecular information and communication science. Proc. IEEE 2016, 105, 306-318. [CrossRef] 
399. Katz, M.; Matinmikko-Blue, M.; Latva-Aho, M. 6Genesis flagship program: Building the bridges towards 6G-enabled wireless smart society and ecosystem. In Proceedings of the 2018 IEEE 10th Latin-American Conference on Communications (LATINCOM), Guadalajara, Mexico, 14-16 November 2018; pp. 1-9.

400. Andrews, J.G.; Buzzi, S.; Choi, W.; Hanly, S.V.; Lozano, A.; Soong, A.C.; Zhang, J.C. What will 5G be? IEEE J. Sel. Areas Commun. 2014, 32, 1065-1082. [CrossRef]

401. Hawilo, H.; Shami, A.; Mirahmadi, M.; Asal, R. NFV: State of the art, challenges, and implementation in next generation mobile networks (vEPC). IEEE Netw. 2014, 28, 18-26. [CrossRef]

402. Miyazawa, M.; Hayashi, M.; Stadler, R. vNMF: Distributed fault detection using clustering approach for network function virtualization. In Proceedings of the 2015 IFIP/IEEE International Symposium on Integrated Network Management (IM), Ottawa, ON, Canada, 11-15 May 2015; pp. 640-645.

403. Bolla, R.; Lombardo, C.; Bruschi, R.; Mangialardi, S. DROPv2: Energy efficiency through network function virtualization. IEEE Netw. 2014, 28, 26-32. [CrossRef]

404. Quittek, J.; Bauskar, P.; BenMeriem, T.; Bennett, A.; Besson, M. Network functions virtualisation (nfv)-management and orchestration. In ETSI NFV ISG; White Paper; European Telecommunications Standards Institute: Sophia Antipolis, France, 2014; pp. 733-8716.

405. Mahdi, M.N.; Mohamed, K.S.; Ahmad, A.R.; Subhi, M.A. The Vision of 5G and Cell-Free Communication Networks in Malaysia. In Proceedings of the 2020 8th International Conference on Information Technology and Multimedia (ICIMU), Selangor, Malaysia, 24-26 August 2020; pp. 156-161. 